JOURNAL OF THE

AMERICAN MATHEMATICAL SOCIETY

Volume 17, Number 3, Pages 679-722

S 0894-0347(04)00455-2

Article electronically published on April 1, 2004

\title{
ON THE NONVANISHING OF THE CENTRAL VALUE OF THE RANKIN-SELBERG $L$-FUNCTIONS
}

\author{
DAVID GINZBURG, DIHUA JIANG, AND STEPHEN RALLIS
}

Dedicated to Ilya I. Piatetski-Shapiro with admiration on the occasion of his 75th birthday

\section{INTRODUCTION}

Let $\pi_{1}$ and $\pi_{2}$ be irreducible unitary cuspidal automorphic representations of $\mathrm{GL}_{m}(\mathbb{A})$ and $\mathrm{GL}_{n}(\mathbb{A})$, respectively, where $\mathbb{A}$ is the ring of adeles attached to a number field $k$. The basic analytic properties (meromorphic continuation and the functional equation) of the Rankin-Selberg $L$-functions $L\left(s, \pi_{1} \times \pi_{2}\right)$ have been established through the work JPSS83, [CPS04 and also Shd88 and MW89. It is known from Shd84 that the $L$-functions $L\left(s, \pi_{1} \times \pi_{2}\right)$ defined by the RankinSelberg method and by the Langlands-Shahidi method are the same.

The problems related to the value of the (complete) Rankin-Selberg $L$-function $L\left(s, \pi_{1} \times \pi_{2}\right)$ at the center of symmetry (i.e., $\left.s=\frac{1}{2}\right)$ are often very interesting. For instance, when both $\pi_{1}$ and $\pi_{2}$ are self-dual, the central value $L\left(\frac{1}{2}, \pi_{1} \times \pi_{2}\right)$ is expected to be nonnegative. See Lp03 and LR03 for a recent account of this problem and see IS00 for relations of this problem to analytic number theory. We are interested in characterizing the nonvanishing of the central value of the Rankin-Selberg $L$-functions $L\left(s, \pi_{1} \times \pi_{2}\right)$ in terms of the nonvanishing of certain period integrals.

Using the global integral representation of $L\left(s, \pi_{1} \times \pi_{2}\right)$ ( CPS04), the nonvanishing of $L\left(\frac{1}{2}, \pi_{1} \times \pi_{2}\right)$ is expected to be equivalent to the nonvanishing of certain period integrals of automorphic forms in $\pi_{1}$ and $\pi_{2}$ over a general linear group. However, as remarked in [H94. §5], one likes to relate the central value of $L$-functions to periods of an arithmetic nature. From the Langlands principle of functoriality and the philosophy of the relative trace formula method, we search for periods which are potentially arithmetic and related to the central value of $L\left(\frac{1}{2}, \pi_{1} \times \pi_{2}\right)$. It will be a very interesting problem to look for integral representations of automorphic $L$ functions based on period integrals of an arithmetic nature ([H94, §5]). This should lead to expressions of the central values of automorphic $L$-functions in terms of the relevant periods which have much deeper arithmetic implications. From this perspective, our study in this paper can be viewed as a preliminary step towards such intrinsic relations between arithmetic geometry and automorphic forms.

Received by the editors May 8, 2003.

2000 Mathematics Subject Classification. Primary 11F67, 11F70, 22E46, 22 E55.

Key words and phrases. Special value, $L$-function, period, automorphic form.

The second author is partially supported by the NSF grant DMS-0098003, the Sloan Research Fellowship, and the McKnight Land-Grant Professorship (University of Minnesota). 
In order to explain our work in a precise way, we recall the Langlands functorial lifts from the classical groups to the general linear group. Let $\pi$ be an irreducible unitary cuspidal automorphic representation of $\mathrm{GL}_{n}(\mathbb{A})$. Then the Rankin-Selberg $L$-function $L(s, \pi \times \pi)$ has a pole at $s=1$ if and only if $\pi \cong \pi^{\vee}$, the contragredient of $\pi$. In this case the pole is simple. Now assume that $\pi$ is self-dual; i.e., $\pi \cong \pi^{\vee}$. We have

$$
L(s, \pi \times \pi)=L\left(s, \pi, \Lambda^{2}\right) \cdot L\left(s, \pi, \mathrm{Sym}^{2}\right)
$$

where $L\left(s, \pi, \Lambda^{2}\right)$ is the exterior square $L$-function and $L\left(s, \pi, \mathrm{Sym}^{2}\right)$ is the symmetric square $L$-function, attached to $\pi$ respectively. It follows that for any given irreducible unitary self-dual cuspidal automorphic representation of $\mathrm{GL}_{n}(\mathbb{A})$, one and only one of the two $L$-functions $L\left(s, \pi, \Lambda^{2}\right)$ and $L\left(s, \pi, \mathrm{Sym}^{2}\right)$ has a simple pole at $s=1$. If the exterior square $L$-function $L\left(s, \pi, \Lambda^{2}\right)$ has a simple pole at $s=1$ (which implies that $n$ is even $K 00$ ), we say that $\pi$ is symplectic, and if the symmetric square $L$-function $L\left(s, \pi, \mathrm{Sym}^{2}\right)$ has a simple pole at $s=1$, we say that $\pi$ is orthogonal. This terminology is clearly compatible with the Langlands functorial principle and with the recent work on the Langlands functorial lifts from irreducible generic cuspidal automorphic representations of classical groups to the general linear group ([CKPSS01], CKPSS], GRS99c, GRS01, S02, and JS04]). We say that an irreducible cuspidal automorphic representation is generic if it has a nonzero (global) Whittaker-Fourier coefficient with respect to a generic character.

The following is the main consequence of the automorphic descent constructions in GRS99c, GRS01, and S02.

Theorem (Ginzburg-Rallis-Soudry). Let $\pi$ be an irreducible unitary self-dual cuspidal automorphic representation of $\mathrm{GL}_{n}(\mathbb{A})$. If the exterior square L-function $L\left(s, \pi, \Lambda^{2}\right)$ has a pole at $s=1$, then there exists an irreducible generic cuspidal automorphic representation $\sigma$ of $\mathrm{SO}_{2 r+1}(\mathbb{A})(n=2 r)$ such that $\pi$ is a weak Langlands functorial lift from $\sigma$. If the symmetric square $L$-function $L\left(s, \pi, \mathrm{Sym}^{2}\right)$ has a pole at $s=1$, then if $n=2 l$ is even, there exists an irreducible generic cuspidal automorphic representation $\sigma$ of $\mathrm{SO}_{2 l}(\mathbb{A})$ such that $\pi$ is a weak Langlands functorial lift from $\sigma$; and if $n=2 l+1$ is odd, there exists an irreducible generic cuspidal automorphic representation $\sigma$ of $\mathrm{Sp}_{2 l}(\mathbb{A})$ such that $\pi$ is a weak Langlands functorial lift from $\sigma$.

We recall that $\pi$ is a weak lift from $\sigma$ means by definition that at almost all local places, the local component of $\pi$ is a local Langlands functorial lift from the corresponding local component of $\sigma$. Now it is known that the weak lifts in the theorem are strong; i.e., it is compatible with the local Langlands functorial lift at every local place (see [JS04, [K02 for $\mathrm{SO}_{2 n+1}$ and CKPSS for split classical groups). From now on we simply do not distinguish the Langlands functorial lift from the weak functorial lift. We remark that by the local converse theorem (which has been established by D. Jiang and D. Soudry for $\mathrm{SO}_{2 r+1}$ ([JS03] and [JS04]) and which is their work in progress for other classical groups), one can prove that the representation $\sigma$ given in the theorem is uniquely determined by $\pi$. From the above theorem one deduces

Corollary. Let $\pi$ be an irreducible unitary self-dual cuspidal automorphic representation of $\mathrm{GL}_{n}(\mathbb{A})$. If $\pi$ is symplectic, then $n=2 r$ is even and $\pi$ is the functorial lift from an irreducible generic cuspidal automorphic representation $\sigma$ of $\mathrm{SO}_{2 r+1}(\mathbb{A})$. If $\pi$ is orthogonal, then if $n=2 l$ is even, $\pi$ is a functorial lift from an irreducible 
generic cuspidal automorphic representation $\sigma$ of $\mathrm{SO}_{2 l}(\mathbb{A})$; and if $n=2 l+1$ is odd, $\pi$ is a functorial lift from an irreducible generic cuspidal automorphic representation $\sigma$ of $\operatorname{Sp}_{2 l}(\mathbb{A})$.

Let $\pi_{1}$ and $\pi_{2}$ be irreducible unitary self-dual cuspidal automorphic representations of $\mathrm{GL}_{m}(\mathbb{A})$ and of $\mathrm{GL}_{n}(\mathbb{A})$, respectively. When $\pi_{1}$ and $\pi_{2}$ are both orthogonal or both symplectic, the Rankin-Selberg product $L$-function $L\left(s, \pi_{1} \times \pi_{2}\right)$ may have a pole at $s=1$. The existence of the pole of $L\left(s, \pi_{1} \times \pi_{2}\right)$ is equivalent to the property that $\pi_{2} \cong \pi_{1}^{\vee}$. It seems very mysterious to characterize the nonvanishing of the central value of $L\left(s, \pi_{1} \times \pi_{2}\right)$ at $s=\frac{1}{2}$ in this case in terms of period integrals.

Our objective is to provide a characterization for the nonvanishing of the central value of the Rankin-Selberg product $L$-function $L\left(s, \pi_{1} \times \pi_{2}\right)$ when one of the two representations $\pi_{1}$ and $\pi_{2}$ is symplectic and the other one is orthogonal. In terms of the global Langlands reciprocity conjecture, this should be the case where $\pi_{1} \otimes \pi_{2}$ is symplectic, and the previous should be the case where $\pi_{1} \otimes \pi_{2}$ is orthogonal. One may find more detailed discussion on the terminology and the tensor product lift in [R94, §3]. The symplectic case leads to one of the following two cases.

Case 1. $m=2 l+1$ is odd and $\pi_{1}$ is orthogonal; $n=2 r$ is even and $\pi_{2}$ is symplectic.

Case 2. $m=2 l$ is even and $\pi_{1}$ is orthogonal; $n=2 r$ is even and $\pi_{2}$ is symplectic.

In Case 1, we know from the above theorem of Ginzburg-Rallis-Soudry that $\pi_{1}$ is a Langlands functorial lift from an irreducible unitary generic cuspidal automorphic representation $\sigma$ of $\mathrm{Sp}_{2 l}(\mathbb{A})$ and $\pi_{2}$ is a Langlands functorial lift from an irreducible unitary generic cuspidal automorphic representation $\tau$ of $\mathrm{SO}_{2 r+1}(\mathbb{A})$. By the global theta correspondence, we know that if the standard $L$-function $L\left(\frac{1}{2}, \tau\right) \neq 0$, then the representation $\tau$ is a global theta lift (with respect to a given character $\psi$ ) from an irreducible unitary generic cuspidal automorphic representation $\widetilde{\tau}$ of ${\widetilde{\mathrm{Sp}_{2 r}}}_{2}(\mathbb{A})$, where $\widetilde{\mathrm{Sp}}_{2 r}$ is the metaplectic double cover of $\mathrm{Sp}_{2 r}$. In this case, we call $\pi_{2}$ a $\psi$ transfer of $\widetilde{\tau}$ from $\widetilde{\mathrm{Sp}}_{2 r}$ to $\mathrm{GL}_{2 r}$. The main result of this paper is to characterize the nonvanishing of $L\left(\frac{1}{2}, \pi_{1} \times \pi_{2}\right)$ in terms of the nonvanishing of the period attached to either $\left(\sigma, \widetilde{\tau}, \mathrm{Sp}_{2 l}\right)$ if $r \geq l$ or $\left(\sigma, \widetilde{\tau}, \widetilde{\mathrm{Sp}}_{2 r}\right)$ if $l \geq r$. From [F95], one knows that if $L\left(\frac{1}{2}, \tau\right)=0$, it is expected that the global theta lift of $\tau$ to $\widetilde{\mathrm{Sp}}_{2 r}$ should be zero and the theta lift to $\widetilde{\mathrm{Sp}}_{2 r+2}$ is cuspidal. We will not discuss this case here.

In Case 2, $\pi_{1}$ is a functorial lift from an irreducible unitary generic cuspidal automorphic representation $\sigma$ of $\mathrm{SO}_{2 l}(\mathbb{A})$ and $\pi_{2}$ is a functorial lift from an irreducible unitary generic cuspidal automorphic representation $\tau$ of $\mathrm{SO}_{2 r+1}(\mathbb{A})$. The nonvanishing of $L\left(\frac{1}{2}, \pi_{1} \times \pi_{2}\right)$ will be characterized in terms of the nonvanishing of the period attached to either $\left(\sigma, \tau, \mathrm{SO}_{2 l}\right)$ if $r \geq l$ or $\left(\sigma, \tau, \mathrm{SO}_{2 r+1}\right)$ if $l>r$. By the Langlands functoriality from generic cuspidal automorphic representations of special orthogonal groups to the general linear groups ([CKPSS]), one might define

$$
L(s, \sigma \times \tau):=L\left(s, \pi_{1} \times \pi_{2}\right) .
$$

Then the assertion on the characterization of the nonvanishing of the central value $L\left(\frac{1}{2}, \sigma \times \tau\right)$ in terms of periods attached to either $\left(\sigma, \tau, \mathrm{SO}_{2 l}\right)$ if $r \geq l$ or $\left(\sigma, \tau, \mathrm{SO}_{2 r+1}\right)$ if $l>r$ is a conjecture of Gross and Prasad (see GP92 and GP94 for the global conjecture when $r=l$ or $l-1$ and for the local conjecture in general). When $r=1$ and $l=1$, the assertion was proved by Waldspurger in W85. When $l=2$ and $r=1$, it is a conjecture of Jacquet on the relation between the nonvanishing 
of the central value of the triple product $L$-function and the nonvanishing of the trilinear periods. For the split period case, it was proved in Jng98b and Jnng01. For general period cases, it was proved completely in HK91 and [HK04. When $r=2$ and $l=2$, some special cases were studied in [HK92] and BFSP04. In general this will be the subject matter of our work [GJR], the detail of which will be omitted here.

The main results of this paper (dealing with Case 1) can be formulated as follows.

Main Theorem. Let $\pi_{1}$ be an irreducible unitary cuspidal orthogonal automorphic representation of $\mathrm{GL}_{2 l+1}(\mathbb{A})$, and let $\pi_{2}$ be an irreducible unitary cuspidal automorphic symplectic representation of $\mathrm{GL}_{2 r}(\mathbb{A})$. Assume that the standard L-function $L\left(\frac{1}{2}, \pi_{2}\right) \neq 0$. Let $\sigma$ be an irreducible unitary generic cuspidal automorphic representation of $\mathrm{Sp}_{2 l}(\mathbb{A})$ which lifts functorially to $\pi_{1}$ and let $\widetilde{\tau}$ be an irreducible unitary generic cuspidal automorphic representation of $\widetilde{\operatorname{Sp}}_{2 r}(\mathbb{A})$ which has the $\psi$-transfer $\pi_{2}$.

(1) (Theorem 5.1) If the period integral

$$
\mathcal{P}_{r, r-l}\left(\phi_{l}, \widetilde{\phi}_{r}, \varphi_{l}\right)(r \geq l) \quad \text { or } \quad \widetilde{\mathcal{P}}_{l, l-r}\left(\widetilde{\phi}_{r}, \phi_{l}, \varphi_{r}\right)(r \leq l)
$$

attached to $(\sigma, \widetilde{\tau}, \psi)$ (see $\S 2$ for an explicit definition) is nonzero for some choice of data, then the central value of the Rankin-Selberg product $L$ function $L\left(\frac{1}{2}, \pi_{1} \times \pi_{2}\right)$ is nonzero.

(2) (Theorem 6.3) Assume that Assumption (FC) (see the remark below) hold for the pair $(\widetilde{\tau}, \sigma)$. If the central value of the Rankin-Selberg product $L$-function $L\left(\frac{1}{2}, \pi_{1} \times \pi_{2}\right)$ is nonzero, then there exist an irreducible unitary generic cuspidal automorphic representation $\sigma_{1}$ of $\mathrm{Sp}_{2 l}(\mathbb{A})$ which is nearly equivalent to $\sigma$ and an irreducible unitary cuspidal automorphic representation $\widetilde{\tau}_{1}$ of $\widetilde{\mathrm{Sp}}_{2 r}(\mathbb{A})$ which is nearly equivalent to $\widetilde{\tau}$, such that the period integral

$$
\mathcal{P}_{r, r-l}\left(\phi_{l}, \widetilde{\phi}_{r}, \varphi_{l}\right)(r \geq l) \quad \text { or } \quad \widetilde{\mathcal{P}}_{l, l-r}\left(\widetilde{\phi}_{r}, \phi_{l}, \varphi_{r}\right)(r \leq l)
$$

attached to $\left(\sigma_{1}, \widetilde{\tau}_{1}, \psi\right)$ (see $§ 2$ for an explicit definition) is nonzero for some choices of data.

Remark. First, the periods considered in the above theorem are generalized Gelfand-Graev model integrals and are called periods of Fourier-Jacobi type, which will be explicitly defined in $\S 2$. The nonvanishing of the periods implies the implicit relation between the generic character for $\sigma$ and the character $\psi$ for the $\psi$-transfer of $\widetilde{\tau}$. Secondly, part (2) of the above theorem has been proved based on Assumption (FC) which is about the nonvanishing of certain Fourier coefficients of a residual representation attached to the pair $\left(\sigma, \pi_{2}\right)$ and will be discussed in detail in $\S 6$. In $\S 7$, we verify Assumption (FC) for the case of $l=1$, while for the case $l=0$, it is proved in GRS99a. In other words, part (2) of the above theorem has been completed for the case $r \geq l=1,0$. Finally, it is expected that $\widetilde{\tau}_{1}$ in part (2) is generic, but we can prove it only when Conjecture 6.1 holds.

The paper is organized as follows. In $\S 2$, we introduce the notation which will be used in the rest of the paper. In $\S 3$, we recall the basic facts of Eisenstein series and determine the location of poles of Eisenstein series. $\S 4$ starts with the study of periods of residues of Eisenstein series, which needs Arthur's truncation 
method to justify the convergence. We provide the details for the case $r \geq l$. The other case is treated similarly. The main result here is Theorem 4.4, which is an identity relating the 'outer' period of the residues of an Eisenstein series to the 'inner' period of the cuspidal datum of the Eisenstein series. The idea was used to study the nonvanishing of the central value of the third symmetric power $L$-function of $\mathrm{GL}_{2}$ in GJR01, which is a similar, but lower rank, case. In general such an idea has been used to treat many cases in JR92, FJ93, Jng98a, JLR04, GJR03 and GJR. In $\S 5$, we prove part (1) of the Main Theorem, which is Theorem 5.1, by using the same argument as in GJR01. $\S 6$ treats part (2) of the Main Theorem, which is Theorem 6.3. Here we use some ideas from the nonvanishing of Fourier coefficients of automorphic forms attached to unipotent orbits GRS03] and the construction of automorphic descent maps [GRS]. In $\S 7$, we verify the important Assumption (FC) for the case of $l=1$.

\section{PERIODS}

In this section, we give a formal definition of the periods for automorphic forms used in this paper. The remaining sections are devoted to the study of these periods for automorphic forms occurring in the discrete spectrum.

Let $k$ be a number field and $\mathbb{A}$ the ring of adeles of $k$. Let $\mathrm{Sp}_{2 p}$ be the $k$-split symplectic group of rank $p$, which preserves the symplectic form given inductively by

$$
\mathcal{J}_{2 p}=\left(\begin{array}{ccc}
0 & & 1 \\
& \mathcal{J}_{2 p-2} & \\
-1 & & 0
\end{array}\right) .
$$

Let $\left\{\alpha_{1}, \alpha_{2}, \cdots, \alpha_{p}\right\}$ be the set of simple roots, which determines the Borel subgroup $B=T U$ with $U$ upper-triangular. We have to consider two kinds of parabolic subgroups in two different symplectic groups. To unify the notation we are going to use, we introduce one standard parabolic subgroup

$$
Q_{p, p-i}=L_{p, p-i} V_{p, p-i}
$$

of $\mathrm{Sp}_{2 p}$ with the Levi part $L_{p, p-i}$ isomorphic to $G L_{1}^{i} \times \mathrm{Sp}_{2(p-i)}$. We write the unipotent radical $V_{p, p-i}$ as

$$
V_{p, p-i}=\left\{v(n, x, y, z):=\left(\begin{array}{cccc}
n & x & y & z \\
& I_{p-i} & 0 & y^{*} \\
& 0 & I_{p-i} & x^{*} \\
& & & n^{*}
\end{array}\right) \in \operatorname{Sp}_{2 p}\right\}
$$

where $n \in N_{i}$ is the standard (or upper-triangular) maximal unipotent subgroup of $\mathrm{GL}_{i}$. When there is no confusion with the indication of $\mathrm{Sp}_{2 p}$, we may simply use

$$
Q_{p-i}=L_{p-i} V_{p-i} .
$$

Another standard parabolic subgroup of $\mathrm{Sp}_{2 p}$, we will consider is denoted by

$$
P_{i, p-i}=M_{i, p-i} U_{i, p-i}
$$

where the Levi part $M_{i, p-i}$ is isomorphic to $\mathrm{GL}_{i} \times \operatorname{Sp}_{2(p-i)}$. Here the indices $\{i, p-i\}$ form a partition of the rank $p$ of $\mathrm{Sp}_{2 p}$. Since there will be two different symplectic groups occurring in the rest of the paper, we use such notation to avoid unnecessary confusion. 
It is clear from the definition that $V_{p-i+1}$ is a normal subgroup in $V_{p-i}$. Let $\ell_{p-i}=\ell_{p, p-i}$ be the projection from $V_{p-i}$ onto the quotient $V_{p-i} / V_{p-i+1}$, which is the Heisenberg group $H_{p-i}$ of dimension $2(p-i)+1$. We may also identify $\ell_{p-i}\left(V_{p-i}\right)$ with the subgroup (section) in $V_{p-i}$, which has the form

$$
\left\{\ell_{p-i}(v)=\left(\begin{array}{cccccc}
I_{i-1} & & & & & \\
& 1 & \bar{x} & \bar{y} & \bar{z} & \\
& & I_{p-i} & 0 & \bar{y}^{*} & \\
& & & I_{p-i} & \bar{x}^{*} & \\
& & & & 1 & \\
& & & & & I_{i-1}
\end{array}\right\} \in \operatorname{Sp}_{2 p}\right\} .
$$

Here $\bar{x}, \bar{y}$ are the $i$-th (the last) row of $x, y$ in (2.1), respectively, and $\bar{z}$ is the $(i, 1)$-th entry of $z$ in $(2.1)$.

For a given nontrivial character $\psi$ of $k$, define $\psi_{i}$ to be the generic character of $N_{i}$ of the form

$$
\psi_{i}(n):=\psi\left(n_{1,2}+\cdots+n_{i-1, i}\right) .
$$

We also view $\psi_{i}$ as a character of $V_{p-i}$ by composing with the projection

$$
V_{p-i} \rightarrow V_{p-i} / U_{i, p-i} \cong N_{i}
$$

Clearly, $U_{i, p-i}$ is a normal subgroup of $V_{p-i}$.

To motivate the choices of unipotent subgroups above and the characters below, one has to consider the unipotent conjugacy classes in $\operatorname{Sp}_{2 p}(\mathbb{C})$, where $\mathbb{C}$ is the field of complex numbers. It is known that the conjugacy classes of unipotent elements (often being called unipotent classes or unipotent orbits) of $\mathrm{Sp}_{2 p}(\mathbb{C})$ are parameterized by the partitions with the property that the odd parts of the partitions occur with even multiplicity (Theorem 5.1.3 in CM93]). We will simply call such partitions symplectic partitions. The set of all unipotent (adjoint) orbits of $\mathrm{Sp}_{2 p}(\mathbb{C})$ is denoted by $\mathcal{U}\left(\mathrm{Sp}_{2 p}\right)$ or simply by $\mathcal{U}$. Let $\mathcal{O}_{p, i}$ be the unipotent orbit attached to the symplectic partition $\left((2 i) 1^{2(p-i)}\right)$. We define $V_{\mathcal{O}_{p, i}}$ to be the normal subgroup of $V_{p-i}$ of the form

$$
V_{\mathcal{O}_{p, i}}=\left\{v(n, x, y, z) \in V_{p-i} \mid x_{i, j}=y_{i, j}=0,1 \leq j \leq p-i\right\}
$$

For any $a \in k^{\times}$, we define a character $\psi_{\mathcal{O}_{p, i}}^{a}$ of $V_{\mathcal{O}_{p, i}}$ to be

$$
\psi_{\mathcal{O}_{p, i}}^{a}(v)=\psi_{i}(n) \psi\left(a z_{i, 1}\right)=\psi_{i, a}(v)
$$

where $v=v(n, x, y, z) \in V_{p-i}$ and $z_{i, 1}$ is the $(i, 1)$-th entry of $z$. It is easy to check that the character $\psi_{\mathcal{O}_{p, i}}^{a}$ is the one corresponding to a $k$-rational orbit in $\mathcal{O}_{p, i}$. (See MW87, where the unipotent subgroup $V_{\mathcal{O}_{p, i}}$ is denoted by $U^{\prime \prime}$.)

Let $\widetilde{\operatorname{Sp}}_{2 q}(\mathbb{A})$ be the metaplectic double cover of $\operatorname{Sp}_{2 q}(\mathbb{A})$. We follow GRS02] and I94 for the discussion of basic structures and representations of $\widetilde{\operatorname{Sp}}_{2 q}(\mathbb{A})$ and the related Jacobi groups. We consider the (adelic) Jacobi group

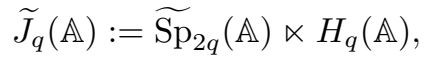


where $H_{q}$ is the Heisenberg group of dimension $2 q+1$. We shall denote elements of $H_{q}$ by

$$
\left(a_{1}, \cdots, a_{q} ; b_{1}, \cdots, b_{q} ; c\right):=\left(\begin{array}{cccc}
1 & a & b & c \\
& I_{q} & 0 & b^{*} \\
& 0 & I_{q} & a^{*} \\
& & & 1
\end{array}\right) \in \operatorname{Sp}_{2 q+2}
$$

where $a=\left(a_{1}, \cdots, a_{q}\right)$ and $b=\left(b_{1}, \cdots, b_{q}\right)$. The symplectic group $\mathrm{Sp}_{2 q}$ is embedded in $\mathrm{Sp}_{2 q+2}$ by

$$
g \mapsto\left(\begin{array}{ccc}
1 & & \\
& g & \\
& & 1
\end{array}\right) .
$$

For a given character $\psi$ of $\mathbb{A} / k$, there is a unique, up to equivalence, irreducible representation $\omega_{\psi}$ of $H_{q}(\mathbb{A})$, which is realized in in the space of Bruhat-Schwartz functions $\mathcal{S}\left(\mathbb{A}^{q}\right)$, by the Stone-Von Neumann Theorem in Chapter II of [MVW87. We identify here elements $\left(a_{1}, \cdots, a_{q}\right)$ of $k^{q}$ with $\left(a_{1}, \cdots, a_{q} ; 0, \cdots, 0 ; 0\right)$ of $H_{q}$. One can extend $\omega_{\psi}$ to the Weil representation $\omega_{\psi}$ of $\widetilde{J}_{q}(\mathbb{A})$ ([94]) and define the theta function $\widetilde{\theta}_{\varphi_{q}}^{\psi}(h \widetilde{g})$ by

$$
\widetilde{\theta}_{\varphi_{q}}^{\psi}(h \widetilde{g})=\sum_{\xi \in k^{q}} \omega_{\psi}(h \widetilde{g}) \varphi_{q}(\xi)
$$

where $\varphi_{q} \in \mathcal{S}\left(\mathbb{A}^{q}\right)$.

Let $\phi_{p}$ be an automorphic form on $\operatorname{Sp}_{2 p}(\mathbb{A})$. We define the (generalized) FourierJacobi coefficient of $\phi_{p}$ ([194], GRS99c]) by

$$
\widetilde{\mathcal{F}}_{\varphi_{p-i}}^{\psi}\left(\phi_{p}\right)(\widetilde{g}):=\int_{V_{p-i}(k) \backslash V_{p-i}(\mathbb{A})} \widetilde{\theta}_{\varphi_{p-i}}^{\psi}\left(\ell_{p-i}(v) \widetilde{g}\right) \phi_{p}(v \widetilde{g}) \psi_{i}(v) d v .
$$

Note that because of the nature of the Weil representations, the automorphic form $\phi_{p}$ on $\operatorname{Sp}_{2 p}(\mathbb{A})$, after integrating over $V_{p-i}(k) \backslash V_{p-i}(\mathbb{A})$, becomes an automorphic form on $\widetilde{S p}_{2(p-i)}(\mathbb{A})$. This can be observed clearly from the discussion below on the relation between (2.8) and the Fourier-Jacobi coefficients defined in I94.

Recall from (2.2) that $V_{p-i+1}$ is a normal subgroup of $V_{p-i}$ and the projection $\ell_{p-i}\left(V_{p-i}\right)$ can be identified with the subgroup $H_{p-i}$ given in (2.2). Note that $N_{i}$ is a subgroup of $V_{p-i+1}$, so that the generic character $\psi_{i}$ defined in (2.3) can be viewed as a character of $V_{p-i+1}$. Consider the $\psi_{i}$ Fourier coefficient of $\phi_{p}$

$$
\phi_{p}^{\psi_{i}}(h g)=\int_{V_{p-i+1}(k) \backslash V_{p-i+1}(\mathbb{A})} \phi_{p}(u h g) \psi_{i}(u) d u
$$

where $h \in H_{p-i}(\mathbb{A})$ and $g \in \operatorname{Sp}_{2(p-i)}(\mathbb{A})$. It is easy to check that $\phi_{p}^{\psi_{i}}(h g)$ is automorphic on the group $H_{p-i}(\mathbb{A}) \ltimes \operatorname{Sp}_{2(p-i)}(\mathbb{A})$. Now we have

$$
\widetilde{\mathcal{F}}_{\varphi_{p-i}}^{\psi}\left(\phi_{p}\right)(\widetilde{g})=\int_{H_{p-i}(k) \backslash H_{p-i}(\mathbb{A})} \widetilde{\theta}_{\varphi_{p-i}}^{\psi}(h \widetilde{g}) \phi_{p}^{\psi_{i}}(h \widetilde{g}) d h .
$$

By [I94] and [GRS99c], $\widetilde{\mathcal{F}}_{\varphi_{p-i}}^{\psi_{p}}\left(\phi_{p}\right)(\widetilde{g})$ is a Fourier-Jacobi coefficient of $\phi_{p}^{\psi_{i}}$ and is an automorphic form on $\widetilde{\operatorname{Sp}}_{2(p-i)}(\mathbb{A})$. For simplicity, we call $\widetilde{\mathcal{F}}_{\varphi_{p-i}}^{\psi}\left(\phi_{p}\right)(\widetilde{g})$ a FourierJacobi coefficient of $\phi_{p}$. 
Let $\widetilde{\phi}_{q}$ be an automorphic form on $\widetilde{\mathrm{Sp}}_{2 q}(\mathbb{A})$. We define similarly the FourierJacobi coefficient of $\widetilde{\phi}_{q}$ by

$$
\mathcal{F}_{\varphi_{q-i}}^{\psi}\left(\widetilde{\phi}_{q}\right)(g):=\int_{V_{q-i}(k) \backslash V_{q-i}(\mathbb{A})} \widetilde{\theta}_{\varphi_{q-i}}^{\psi}\left(\ell_{q-i}(v) \widetilde{g}\right) \widetilde{\phi}_{q}(v \widetilde{g}) \psi_{i}(v) d v
$$

which is an automorphic form on $\operatorname{Sp}_{2(q-i)}(\mathbb{A})$. Note here that $\widetilde{g}$ is a preimage of $g$. Since the product is no longer genuine, it is independent of the choice of the preimage $\widetilde{g}$ for any $g$.

Let $\widetilde{\phi}_{p-i}$ be an automorphic form on $\widetilde{\operatorname{Sp}}_{2(p-i)}(\mathbb{A})$ and let $\phi_{q-i}$ be an automorphic form on $\operatorname{Sp}_{2(q-i)}(\mathbb{A})$. We formally define two period integrals:

$$
\widetilde{\mathcal{P}}_{p, i}\left(\widetilde{\phi}_{p-i}, \phi_{p}, \varphi_{p-i}\right):=\int_{\operatorname{Sp}_{2(p-i)}(k) \backslash \operatorname{Sp}_{2(p-i)}(\mathbb{A})} \widetilde{\phi}_{p-i}(\widetilde{g}) \widetilde{\mathcal{F}}_{\varphi_{p-i}}^{\psi}\left(\phi_{p}\right)(\widetilde{g}) d g
$$

and

$$
\mathcal{P}_{q, i}\left(\phi_{q-i}, \widetilde{\phi}_{q}, \varphi_{q-i}\right):=\int_{\operatorname{Sp}_{2(q-i)}(k) \backslash \operatorname{Sp}_{2(q-i)}(\mathbb{A})} \phi_{q-i}(g) \mathcal{F}_{\varphi_{q-i}}^{\psi}\left(\widetilde{\phi}_{q}\right)(g) d g .
$$

Note in $(2.12)$ that $\widetilde{g}$ is any preimage of $g$ in $\widetilde{\mathrm{Sp}}_{2(p-i)}(\mathbb{A})$. This makes sense because the product of two genuine automorphic forms over the metaplectic cover $\widetilde{\mathrm{Sp}}_{2(p-i)}(\mathbb{A})$ is no longer genuine, and hence the integration in (2.12) is taken over the linear group.

It is clear that if $\widetilde{\phi}_{p-i}$ (or $\phi_{q-i}$, respectively) is a cuspidal automorphic form, then the period $\widetilde{\mathcal{P}}_{p, i}\left(\widetilde{\phi}_{p-i}, \phi_{p}, \varphi_{p-i}\right)$ (or $\mathcal{P}_{q, i}\left(\phi_{q-i}, \widetilde{\phi}_{q}, \varphi_{q-i}\right)$, respectively) is well defined; i.e., the integral converges absolutely. However, in general, we have to justify the convergence problem of these integrals.

Let $\left(\sigma, V_{\sigma}\right)$ be an irreducible unitary generic cuspidal automorphic representation of $\operatorname{Sp}_{2 l}(\mathbb{A})$ and let $\left(\widetilde{\tau}, V_{\widetilde{\tau}}\right)$ be an irreducible unitary generic cuspidal automorphic representation of $\widetilde{\mathrm{Sp}}_{2 r}(\mathbb{A})$. When $p=l$ and $i=l-r(l \geq r)$ or $q=r$ and $i=r-l$ $(r \geq l)$, then the period $\widetilde{\mathcal{P}}_{l, l-r}\left(\widetilde{\phi}_{r}, \phi_{l}, \varphi_{r}\right)(l \geq r)$ or $\mathcal{P}_{r, r-l}\left(\phi_{l}, \widetilde{\phi}_{r}, \varphi_{l}\right)(r \geq l)$ is said to be attached to $(\sigma, \widetilde{\tau}, \psi)$, respectively, if $\phi \in V_{\sigma}$ and $\widetilde{\tau} \in V_{\widetilde{\tau}}$, and $\psi$ is a given nontrivial additive character of $k \backslash \mathbb{A}$.

To simplify the notation, we use

$$
[H]:=H(k) \backslash H(\mathbb{A})
$$

for any algebraic group $H$ defined over $k$. We also use $H$ for the $k$-rational points of $H$ if it does not cause any confusion.

\section{Some families of Residual REPRESEntations}

3.1. Symplectic groups. We consider here the symplectic group $\operatorname{Sp}_{4 r+2 l}(p=2 r+$ $l$ if using the notation in $\S 2$ ) and the family of cuspidal Eisenstein series associated to the standard maximal parabolic subgroup $P_{2 r, l}$ of $\mathrm{Sp}_{4 r+2 l}$. By the definition in $\S 2$, the Levi decomposition $P_{2 r, l}$ is $P_{2 r, l}=M_{2 r, l} \ltimes U_{2 r, l}$ with $M_{2 r, l} \cong \mathrm{GL}_{2 r} \times \mathrm{Sp}_{2 l}$. The elements of $M_{2 r, l}$ will often be written as $m=m(a, b)$ with $(a, b) \in \mathrm{GL}_{2 r} \times \mathrm{Sp}_{2 l}$. For simplicity, we may also write

$$
m=m(a, b) \in \mathrm{GL}_{2 r} \times \mathrm{Sp}_{2 l} .
$$

Let $\left(\pi \otimes \sigma, V_{\pi \otimes \sigma}\right)$ be an irreducible generic unitary cuspidal automorphic representation of $\mathrm{GL}_{2 r} \times \mathrm{Sp}_{2 l}$. For the given cuspidal datum $\left(P_{2 r, l}, \pi \otimes \sigma\right)$, one may attach 
an Eisenstein series $E\left(g, s, \phi_{\pi \otimes \sigma}\right)$ on $\operatorname{Sp}_{4 r+2 l}(\mathbb{A})$ with $\phi_{\pi \otimes \sigma} \in V_{\pi \otimes \sigma}$. More precisely, the cuspidal automorphic representation $\pi \otimes \sigma$ can be realized in the space of square integrable automorphic functions $\mathrm{L}^{2}\left(Z_{M_{2 r, l}}(\mathbb{A}) M_{2 r, l}(k) \backslash M_{2 r, l}(\mathbb{A})\right)$, where $Z_{M_{2 r, l}}$ is the center of $M_{2 r, l}$. Let $\mathrm{K}$ be the maximal compact subgroup of $\mathrm{Sp}_{4 r+2 l}$ such that

$$
\mathrm{Sp}_{4 r+2 l}(\mathbb{A})=P_{2 r, l}(\mathbb{A}) \mathrm{K}
$$

is the Iwasawa decomposition. Let $\phi_{\pi \otimes \sigma}$ be a $\mathrm{K} \cap M_{2 r, l}(\mathbb{A})$-finite automorphic form in $V_{\pi \otimes \sigma}$, which is extended as a function of $\mathrm{Sp}_{4 r+2 l}(\mathbb{A})$ ([Shd88, §2]), so that for $g=u m \mathrm{k} \in \mathrm{Sp}_{4 r+2 l}(\mathbb{A})$

$$
\phi_{\pi \otimes \sigma}(g)=\phi_{\pi \otimes \sigma}(m \mathrm{k})
$$

and for any fixed $\mathrm{k} \in \mathrm{K}$, the function

$$
m \mapsto \phi_{\pi \otimes \sigma}(m \mathrm{k})
$$

is a $\mathrm{K} \cap M_{2 r, l}(\mathbb{A})$-finite automorphic form in $V_{\pi \otimes \sigma}$. We define

$$
\Phi\left(g, s, \phi_{\pi \otimes \sigma}\right):=\phi_{\pi \otimes \sigma}(g) \exp \left\langle s+\rho_{P_{2 r, l}}, H_{P_{2 r, l}}(g)\right\rangle
$$

for $g \in \operatorname{Sp}_{4 r+2 l}(\mathbb{A})$. As in [Shd88, $\left.\S 1\right]$, the parameter $s$ is identified with $s \tilde{\alpha}_{2 r}$, where $\tilde{\alpha}_{2 r}$ is the co-root dual to the simple root $\alpha_{2 r}$. Note that $\alpha_{2 r}$ determines the standard parabolic subgroup $P_{2 r, l}$.

In our case we have

$$
\exp \left\langle s+\rho_{P_{2 r, l}}, H_{P_{2 r, l}}(g)\right\rangle=|\operatorname{det} a|^{s+\frac{2 r+2 l+1}{2}}
$$

where we write $g=u m(a, b) \mathrm{k} \in \mathrm{Sp}_{4 r+2 l}(\mathbb{A})$ and $m(a, b) \in \mathrm{GL}_{2 r} \times \mathrm{Sp}_{2 l}$. Then the Eisenstein series is given by

$$
E\left(g, s, \phi_{\pi \otimes \sigma}\right)=\sum_{\gamma \in P_{2 r, l}(k) \backslash \mathrm{Sp}_{4 r+2 l}(k)} \Phi\left(\gamma g, s, \phi_{\pi \otimes \sigma}\right) .
$$

The constant term of the Eisenstein series $E\left(g, s, \phi_{\pi \otimes \sigma}\right)$ along a standard parabolic subgroup $P$ is always zero unless $P=P_{2 r, l}$ ([MW95, II.1.7]). Because of the cuspidal datum $\pi \otimes \sigma$ in this case, one has

$$
\begin{aligned}
E_{P_{2 r, l}}\left(g, s, \phi_{\pi \otimes \sigma}\right) & =\int_{\left[U_{2 r, l}\right]} E\left(u g, s, \phi_{\pi \otimes \sigma}\right) d u \\
& =\Phi\left(g, s, \phi_{\pi \otimes \sigma}\right)+\mathcal{M}\left(w_{2 r, l}, s\right)\left(\Phi\left(\cdot, s, \phi_{\pi \otimes \sigma}\right)\right)(g)
\end{aligned}
$$

where $\left[U_{2 r, l}\right]=U_{2 r, l}(k) \backslash U_{2 r, l}(\mathbb{A})$ (as in (2.14)). We denote here by $w_{2 r, l}$ the longest Weyl element in the representatives of the double coset decomposition $W_{M_{2 r, l}} \backslash W_{\mathrm{Sp}_{4 r+2 l}} / W_{M_{2 r, l}}$ of the Weyl groups. The intertwining operator $\mathcal{M}\left(w_{2 r, l}, s\right)$ is defined by the following integral

$$
\mathcal{M}\left(w_{2 r, l}, s\right)\left(\Phi\left(\cdot, s, \phi_{\pi \otimes \sigma}\right)\right)(g):=\int_{\left[U_{2 r, l}\right]} \Phi\left(w_{2 r, l}^{-1} u g, s, \phi_{\pi \otimes \sigma}\right) d u
$$

which is an $\operatorname{Sp}_{4 r+2 l}(\mathbb{A})$-mapping from the unitarily induced representation

$$
\mathrm{I}(s, \pi \otimes \sigma)=\operatorname{Ind}_{P_{2 r, l}(\mathbb{A})}^{\mathrm{Sp}_{4 r+2 l}(\mathbb{A})}\left(\pi \otimes \sigma \otimes \exp \left\langle s, H_{P_{2 r, l}}(\cdot)\right\rangle\right) .
$$

to $I\left(-s, w_{2 r, l}(\pi \otimes \sigma)\right)$.

It follows from the Langlands theory of Eisenstein series that the Eisenstein series $E\left(g, s, \phi_{\pi \otimes \sigma}\right)$ has a pole at $s=s_{0}$ if and only if the term $\mathcal{M}\left(w_{2 r, l}, s\right)\left(\Phi\left(\cdot, s, \phi_{\pi \otimes \sigma}\right)\right)$ in (3.5) has a pole at $s=s_{0}$ for some holomorphic (or standard) section $\Phi\left(g, s, \phi_{\pi \otimes \sigma}\right)$ in $\mathrm{I}(s, \pi \otimes \sigma)$. Since factorizable sections generate a dense subspace in $\mathrm{I}(s, \pi \otimes \sigma)$, it 
suffices to consider the factorizable sections for the existence of poles of the Eisenstein series $E\left(g, s, \phi_{\pi \otimes \sigma}\right)$, or for the existence of poles of $\mathcal{M}\left(w_{2 r, l}, s\right)\left(\Phi\left(\cdot, s, \phi_{\pi \otimes \sigma}\right)\right)$. When the section $\Phi\left(\cdot, s, \phi_{\pi_{2} \otimes \sigma}\right)$ is factorizable; i.e.,

$$
\Phi\left(\cdot, s, \phi_{\pi \otimes \sigma}\right)=\otimes_{v} \Phi_{v}\left(\cdot, s, \phi_{\pi_{v} \otimes \sigma_{v}}\right),
$$

where $\Phi_{v}\left(\cdot, s, \phi_{\pi_{v} \otimes \sigma_{v}}\right)$ is a section in $\mathrm{I}\left(s, \pi_{v} \otimes \sigma_{v}\right)$ and is unramified at almost all finite local places $v$, the term $\mathcal{M}\left(w_{2 r, l}, s\right)\left(\Phi\left(\cdot, s, \phi_{\pi \otimes \sigma}\right)\right)$ can be expressed as an infinite product

$$
\mathcal{M}\left(w_{2 r, l}, s\right)\left(\Phi\left(\cdot, s, \phi_{\pi \otimes \sigma}\right)\right)=\prod_{v} \mathcal{M}_{v}\left(w_{2 r, l}, s\right)\left(\Phi_{v}\left(\cdot, s, \phi_{\pi_{v} \otimes \sigma_{v}}\right)\right) .
$$

By the Langlands-Shahidi theory ([L71], Shd88]) we have

$$
\mathcal{M}\left(w_{2 r, l}, s\right)=\frac{L(s, \pi \times \sigma) L\left(2 s, \pi, \Lambda^{2}\right)}{L(s+1, \pi \times \sigma) L\left(2 s+1, \pi, \Lambda^{2}\right)} \cdot \prod_{v} \mathcal{N}_{v}\left(w_{2 r, l}, s\right),
$$

where $\mathcal{N}_{v}\left(w_{2 r, l}, s\right)$ is the normalized intertwining operator

$$
\mathcal{N}_{v}\left(w_{2 r, l}, s\right)=\frac{1}{r\left(s, \pi_{v}, \sigma_{v}, w_{2 r, l}\right)} \cdot \mathcal{M}_{v}\left(w_{2 r, l}, s\right)
$$

which defines a mapping from $\mathrm{I}\left(s, \pi_{v} \otimes \sigma_{v}\right)$ to $\mathrm{I}\left(-s, w_{2 r, l}\left[\pi_{v} \otimes \sigma_{v}\right]\right)$. Here the function $r\left(s, \pi_{v}, \sigma_{v}, w_{2 r, l}\right)$ is equal to

$$
\frac{L\left(s, \pi_{v} \times \sigma_{v}\right) L\left(2 s, \pi_{v}, \Lambda^{2}\right)}{L\left(s+1, \pi_{v} \times \sigma_{v}\right) L\left(2 s+1, \pi_{v}, \Lambda^{2}\right) \epsilon\left(s, \pi_{v} \times \sigma_{v}, \psi\right) \epsilon\left(2 s, \pi_{v}, \Lambda^{2}, \psi\right)} .
$$

Proposition 3.1 ([K00, Proposition 3.4]). The normalized local intertwining operator $\mathcal{N}_{v}\left(w_{2 r, l}, s\right)$ is holomorphic and nonzero for the real part of s greater than or equal to $\frac{1}{2}$; i.e., for any holomorphic section $\Phi_{v}\left(\cdot, s, \phi_{\pi_{v} \otimes \sigma_{v}}\right)$ in $\mathrm{I}\left(s, \pi_{v} \otimes \sigma_{v}\right)$, as a function in $s, \mathcal{N}_{v}\left(w_{2 r, l}, s\right)\left(\Phi_{v}\left(\cdot, s, \phi_{\pi_{v} \otimes \sigma_{v}}\right)\right)$ is holomorphic and nonzero for the real part of $s$ greater than or equal to $\frac{1}{2}$

Note that $\frac{1}{2}$ can be replaced by 0 in Proposition 3.1 according to Theorem 11.1 in [CKPSS]. But $\frac{1}{2}$ is enough for the work in this paper. As a consequence, one has

Proposition 3.2. The Eisenstein series $E\left(g, s, \phi_{\pi \otimes \sigma}\right)$ can possibly have a simple pole at $s=\frac{1}{2}$ or $s=1$. The existence of the pole at $s=\frac{1}{2}$ or $s=1$ of $E\left(g, s, \phi_{\pi \otimes \sigma}\right)$ is equivalent to the existence of the pole at $s=\frac{1}{2}$ or $s=1$ of the product of L-functions

$$
L(s, \pi \times \sigma) L\left(2 s, \pi, \Lambda^{2}\right),
$$

respectively.

Proof. By the Langlands theory of constant terms of Eisenstein series, the Eisenstein series has a pole at $s=s_{0}$ if and only if the constant terms of the Eisenstein series has a pole at $s=s_{0}$. By (3.5), it is equivalent to the property that the global intertwining operator $\mathcal{M}\left(w_{2 r, l}, s\right)$ has a pole at $s=s_{0} \geq \frac{1}{2}$. From identity (3.9), if the global intertwining operator $\mathcal{M}\left(w_{2 r, l}, s\right)$ has a pole at $s=s_{0}$, then the quotient

$$
\frac{L(s, \pi \times \sigma) L\left(2 s, \pi, \Lambda^{2}\right)}{L(s+1, \pi \times \sigma) L\left(2 s+1, \pi, \Lambda^{2}\right)}
$$

must have a pole at $s=s_{0}$ since, by Proposition 3.1, the product

$$
\prod_{v} \mathcal{N}_{v}\left(w_{2 r, l}, s\right)
$$


does not vanish for $s=s_{0} \geq \frac{1}{2}$. Now both $L$-functions $L(s, \pi \times \sigma)$ and $L\left(s, \pi, \Lambda^{2}\right)$ are nonzero for the real part of $s$ greater than one. It follows that the product

$$
L(s, \pi \times \sigma) L\left(2 s, \pi, \Lambda^{2}\right)
$$

must have a pole at $s=s_{0} \geq \frac{1}{2}$ if $\mathcal{M}\left(w_{2 r, l}, s\right)$ has a pole at $s=s_{0} \geq \frac{1}{2}$. Conversely, if the product of $L$-functions

$$
L(s, \pi \times \sigma) L\left(2 s, \pi, \Lambda^{2}\right)
$$

has a pole at $s=s_{0}$, then the global intertwining operator $\mathcal{M}\left(w_{2 r, l}, s\right)$ has a pole at $s=s_{0}$, because in (3.9), we can always choose a particular factorizable section $\Phi\left(\cdot, s, \phi_{\pi \otimes \sigma}\right)$ as in (3.7), so that the product

$$
\prod_{v} \mathcal{N}_{v}\left(w_{2 r, l}, s\right)\left(\Phi_{v}\left(\cdot, s, \phi_{\pi_{v} \otimes \sigma_{v}}\right)\right)
$$

is holomorphic and nonzero at $s=s_{0}$. Note that for this part we do not need Proposition 3.1.

Finally, by Theorem 7.2 in [CKPSS, the irreducible generic cuspidal automorphic representation $\sigma$ of $\operatorname{Sp}_{2 l}(\mathbb{A})$ has a Langlands functorial lift $\pi(\sigma)$, which is an irreducible unitary automorphic representation of $\mathrm{GL}_{2 l+1}(\mathbb{A})$, is uniquely determined by $\sigma$, and is of isobaric type. Hence one has

$$
L(s, \pi \times \sigma)=L(s, \pi \times \pi(\sigma)) .
$$

It follows that the $L$-function $L(s, \pi \times \sigma)$ is holomorphic and nonvanishing at a real point $s_{0}>1$, and $L(s, \pi \times \sigma)$ has a pole at $s=1$ if and only if $\pi$ is isomorphic to one of the isobaric summands of $\pi(\sigma)$. By Theorem 3.1 in [K00, the complete exterior square $L$-function $L\left(s, \pi, \Lambda^{2}\right)$ is holomorphic and nonvanishing at any real point $s_{0}>1$. If $L\left(s, \pi, \Lambda^{2}\right)$ has a pole at $s=1$, then $i$ is even by Theorem 3.1 in [K00]. By Theorem A in [GRS01], one knows that $L\left(s, \pi, \Lambda^{2}\right)$ has a pole at $s=1$ if and only if $\pi$ is the image of an irreducible unitary generic cuspidal automorphic representation $\tau$ of $\mathrm{SO}_{i+1}(\mathbb{A})$. The uniqueness of $\pi$ in terms of $\tau$ is proved in JS03] and [JS04]. Hence one knows that for the real value $s \geq \frac{1}{2}$, both $L$-functions $L(s, \pi \times \sigma)$ and $L\left(s, \pi, \Lambda^{2}\right)$ can have possible poles only at $s=1$. The proposition follows.

We denote the residue at $s=\frac{1}{2}$ of $E\left(g, s, \phi_{\pi \otimes \sigma}\right)$ by

$$
E_{\frac{1}{2}}\left(g, \phi_{\pi \otimes \sigma}\right):=\operatorname{Res}_{s=\frac{1}{2}} E\left(g, s, \phi_{\pi \otimes \sigma}\right) .
$$

3.2. Metaplectic groups. We consider here the metaplectic group $\widetilde{\operatorname{Sp}}_{6 r}(\mathbb{A})$ and a family of Eisenstein series associated to the standard maximal parabolic subgroup $\widetilde{P}_{2 r, r}(\mathbb{A})$ with Levi decomposition

$$
\widetilde{P}_{2 r, r}(\mathbb{A})=\widetilde{M}_{2 r, r}(\mathbb{A}) \ltimes \widetilde{U}_{2 r, r}(\mathbb{A})=\left(\mathrm{GL}_{2 r}(\mathbb{A}) \times \widetilde{\operatorname{Sp}}_{2 r}(\mathbb{A})\right) \ltimes \widetilde{U}_{2 r, r}(\mathbb{A}) .
$$

Note that the metaplectic cover splits over the $G_{2 r}(\mathbb{A})$-part and the unipotent subgroup $\widetilde{U}_{2 r, r}(\mathbb{A})$, so that we may identity $U_{2 r, r}(\mathbb{A})$ with its preimage in $\widetilde{U}_{2 r, r}(\mathbb{A})$ and $\mathrm{GL}_{2 r}(\mathbb{A})$ with its preimage in $\widetilde{\mathrm{GL}}_{2 r}(\mathbb{A})$.

Let $\pi$ be an irreducible unitary self-dual cuspidal automorphic representation of $\mathrm{GL}_{2 r}(\mathbb{A})$. Assume that $\pi$ is symplectic, i.e., that the exterior square $L$-function 
$L\left(s, \pi, \Lambda^{2}\right)$ has a pole at $s=1$. Let $\widetilde{\tau}$ be an irreducible genuine generic cuspidal automorphic representation of $\widetilde{\mathrm{Sp}}_{2 r}(\mathbb{A})$, so that

$$
\left(\mathrm{GL}_{2 r}(\mathbb{A}) \times{\widetilde{\mathrm{Sp}_{2 r}}}(\mathbb{A}), \pi \otimes \widetilde{\tau}\right)
$$

is a generic cuspidal datum of $\widetilde{\mathrm{Sp}}_{6 r}(\mathbb{A})$. As in the case of $\mathrm{Sp}_{4 r+2 l}$, we form an Eisenstein series $\widetilde{E}\left(\widetilde{g}, s, \widetilde{\phi}_{\pi \otimes \widetilde{\tau}}\right)$ associated to a section $\widetilde{\Phi}\left(\widetilde{g}, s, \widetilde{\phi}_{\pi \otimes \widetilde{\tau}}\right)$ in

$$
\widetilde{I}(s, \pi \otimes \widetilde{\tau}):=\operatorname{Ind}_{\widetilde{P}_{2 r, r}(\mathbb{A})}^{\widetilde{\operatorname{Sp}}_{6 r}(\mathbb{A})}\left(\pi \otimes \widetilde{\tau} \exp \left\langle s, H_{\widetilde{P}_{2 r, r}}(\cdot)\right\rangle\right)
$$

where the parameter $s$ is normalized as in $\S 3.1$, so that

$$
\exp \left\langle s+\rho_{\widetilde{P}_{2 r, r}}, H_{\widetilde{P}_{2 r, r}}(\widetilde{g})\right\rangle=|\operatorname{det} a|^{s+2 r+\frac{1}{2}} .
$$

As in (3.4), one can define an Eisenstein series ([MW95])

$$
\widetilde{E}\left(\widetilde{g}, s, \widetilde{\phi}_{\pi \otimes \tilde{\tau}}\right)=\sum_{\gamma \in \widetilde{P}_{2 r, r}(k) \backslash \widetilde{\operatorname{Sp}}_{6 r}(k)} \widetilde{\Phi}\left(\gamma \widetilde{g}, s, \widetilde{\phi}_{\pi \otimes \tilde{\tau}}\right) .
$$

In this paper we only consider this Eisenstein series for the special case when the irreducible unitary cuspidal automorphic representation $\pi$ of $\mathrm{GL}_{2 r}(\mathbb{A})$ is the $\psi$-transfer of $\widetilde{\tau}$; i.e., $\pi=\pi_{\psi}(\widetilde{\tau})$. It is proved in GRS02 that this $\psi$-transfer enjoys the property that

$$
L^{S}\left(s, \pi_{\psi}(\widetilde{\tau}) \times \rho\right)=L_{\psi}^{S}(s, \widetilde{\tau} \times \rho)
$$

for all irreducible cuspidal automorphic representations $\rho$ of $\mathrm{GL}_{m}(\mathbb{A})$ with all positive integers $m$. Here the set $S$ consists of all infinite local places and the finite local places such that the local components of $\widetilde{\tau}, \rho$ and $\psi$ are unramified at $v \notin S$. Note that the partial $L$-functions for $\widetilde{\tau} \times \rho$ depends on the choice of $\psi$ (GRS02]). If one takes $\rho=\pi_{\psi}(\widetilde{\tau})$, then one knows that the partial $L$-function

$$
L_{\psi}^{S}\left(s, \widetilde{\tau} \times \pi_{\psi}(\widetilde{\tau})\right)
$$

has a simple pole at $s=1$. Since the partial exterior square $L$-function

$$
L^{S}\left(s, \pi_{\psi}(\widetilde{\tau}), \Lambda^{2}\right)
$$

has a simple pole at $s=1$, one knows that the partial symmetric square $L$-function

$$
L^{S}\left(s, \pi_{\psi}(\widetilde{\tau}), \operatorname{Sym}^{2}\right)
$$

is holomorphic and nonzero for the real part of $s$ greater than one.

Following [MW95], the Langlands theory of Eisenstein series works as well for metaplectic groups. This time we can have a formula similar to (3.9), with complete $L$-functions replaced by the partial $L$-functions. The intertwining operator $\widetilde{\mathcal{M}}\left(w_{2 r, r}, s\right)$, which maps from the induced representation $\widetilde{I}\left(s, \pi_{\psi}(\widetilde{\tau}) \otimes \widetilde{\tau}\right)$ to the induced representation $\widetilde{I}\left(-s, w_{2 r, r}\left(\pi_{\psi}(\widetilde{\tau}) \otimes \widetilde{\tau}\right)\right)$, can be expressed as

$$
\begin{aligned}
\widetilde{\mathcal{M}}\left(w_{2 r, r}, s\right) & =\left[r^{S}\left(s, \pi, \sigma, w_{2 r, r}\right) \cdot \prod_{v \notin S} \widetilde{\mathcal{N}}_{v}\left(w_{2 r, r}, s\right)\right] \times \prod_{v \in S} \widetilde{\mathcal{M}}_{v}\left(w_{2 r, r}, s\right), \\
r^{S}\left(s, \pi, \sigma, w_{2 r, r}\right) & =\frac{L_{\psi}^{S}\left(s, \pi_{\psi}(\widetilde{\tau}) \times \widetilde{\tau}\right) L^{S}\left(2 s, \pi_{\psi}(\widetilde{\tau}), \operatorname{Sym}^{2}\right)}{L_{\psi}^{S}\left(s+1, \pi_{\psi}(\widetilde{\tau}) \times \widetilde{\tau}\right) L^{S}\left(2 s+1, \pi_{\psi}(\widetilde{\tau}), \mathrm{Sym}^{2}\right)} .
\end{aligned}
$$


Note that we only consider the normalized local intertwining operators $\widetilde{\mathcal{N}}_{v}\left(w_{2 r, r}, s\right)$ for unramified local places. For unramified local places, the normalization of local intertwining operators is well known and the normalized local intertwining operators are nonzero at the unramified sections. This is the essential difference between this case and the case we considered in $\S 3.1$.

Since one can always choose the local sections $\widetilde{\Phi}_{v}$ in $\widetilde{I}\left(s, \pi_{\psi}\left(\widetilde{\tau}_{v}\right) \otimes \widetilde{\tau}_{v}\right)$ such that the images $\widetilde{\mathcal{M}}_{v}\left(w_{2 r, r}, s\right)\left(\widetilde{\Phi}_{v}\right)$ of $\widetilde{\Phi}_{v}$ are nonzero at $s=1$, it follows that if the partial $L$ function $L_{\psi}^{S}\left(s, \pi_{\psi}(\widetilde{\tau}) \times \widetilde{\tau}\right)$ has a pole at $s=1$, then the global intertwining operator $\widetilde{\mathcal{M}}\left(w_{2 r, r}, s\right)$ has a pole at $s=1$. Hence the Eisenstein series $\widetilde{E}\left(\widetilde{g}, s, \widetilde{\phi}_{\pi \otimes \widetilde{\tau}}\right)$ must have a pole at $s=1$, whose residue is denoted by

$$
\widetilde{E}_{1}\left(\widetilde{g}, \widetilde{\phi}_{\pi \otimes \widetilde{\tau}}\right):=\operatorname{Res}_{s=1} \widetilde{E}\left(\widetilde{g}, s, \widetilde{\phi}_{\pi \otimes \widetilde{\tau}}\right)
$$

for some holomorphic section $\widetilde{\Phi}\left(\widetilde{g}, s, \widetilde{\phi}_{\pi \otimes \tau}\right)$ in $\widetilde{I}(s, \pi \otimes \widetilde{\tau})$.

\section{Periods of Residual Representations}

We recall that $\sigma$ is an irreducible unitary generic cuspidal automorphic representation of $\operatorname{Sp}_{2 l}(\mathbb{A})$ and $\widetilde{\tau}$ is an irreducible unitary genuine generic cuspidal automorphic representation of $\widetilde{\operatorname{Sp}}_{2 r}(\mathbb{A})$. Let $\pi_{\psi}(\widetilde{\tau})$ be the image of $\widetilde{\tau}$ under the $\psi$ transfer with respect to a given character $\psi$, which by definition is the composition of the $\psi$-theta correspondence with $\mathrm{SO}_{2 r+1}$ and the Langlands functorial lifting from $\mathrm{SO}_{2 r+1}$ to $\mathrm{GL}_{2 r}$. The existence and basic properties of this $\psi$-transfer have been proved through CKPSS01], [JS03, JJS04], and [GRS01]. We assume that $\pi_{\psi}(\widetilde{\tau})$ is cuspidal. More results about $\pi_{\psi}(\widetilde{\tau})$ can be found in [S02]. It follows from $\S 3.2$, formula (3.16) in particular, that the residue $\widetilde{E}_{1}\left(\widetilde{h}, \widetilde{\phi}_{\pi_{\psi}(\widetilde{\tau}) \otimes \widetilde{\tau}}\right)$ is nonzero. We are going to study the period integrals of the residues $E_{\frac{1}{2}}\left(g, \phi_{\pi_{\psi}(\widetilde{\tau}) \otimes \sigma}\right)$ in (3.10) and $\widetilde{E}_{1}\left(\widetilde{h}, \widetilde{\phi}_{\pi_{\psi}(\widetilde{\tau}) \otimes \tau}\right)$ in $(3.16)$. Following from the formal definitions of period integrals in (2.12) and (2.13), we have

$$
\begin{array}{ll}
(r \geq l) & \mathcal{P}_{3 r, r-l}\left(E _ { \frac { 1 } { 2 } } \left(\cdot, \phi_{\pi_{\psi}}(\widetilde{\tau}) \otimes \sigma\right.\right. \\
(r \leq l) & \left.\widetilde{\mathcal{P}}_{1}\left(\cdot, \widetilde{\phi}_{\pi_{\psi}(\widetilde{\tau}) \otimes \widetilde{\tau}}\right), \varphi_{2 r+l}\right), \\
(r-r & \left(\widetilde{E}_{1}\left(\cdot, \widetilde{\phi}_{\pi_{\psi}(\widetilde{\tau}) \otimes \widetilde{\tau}}\right), E_{\frac{1}{2}}\left(\cdot, \phi_{\pi_{\psi}(\widetilde{\tau}) \otimes \sigma}\right), \varphi_{3 r}\right) .
\end{array}
$$

We shall only study in detail the case $(r \geq l)$, i.e., the period

$$
\mathcal{P}_{3 r, r-l}\left(E_{\frac{1}{2}}\left(\cdot, \phi_{\pi_{\psi}(\widetilde{\tau}) \otimes \sigma}\right), \widetilde{E}_{1}\left(\cdot, \widetilde{\phi}_{\pi_{\psi}(\widetilde{\tau}) \otimes \widetilde{\tau}}\right), \varphi_{2 r+l}\right)
$$

in the following, and the other case $(r \leq l)$ can be treated in a similar way. By definition, the period in (4.1) is given by the integral

$$
\int_{\left[\mathrm{Sp}_{4 r+2 l}\right]} E_{\frac{1}{2}}\left(g, \phi_{\pi_{\psi}(\widetilde{\tau}) \otimes \sigma}\right) \mathcal{F}_{\varphi_{2 r+l}}^{\psi}\left(\widetilde{E}_{1}\left(\cdot, \widetilde{\phi}_{\pi_{\psi}(\widetilde{\tau}) \otimes \widetilde{\tau}}\right)\right)(g) d g
$$

where $\left[\mathrm{Sp}_{4 r+2 l}\right]=\mathrm{Sp}_{4 r+2 l}(k) \backslash \mathrm{Sp}_{4 r+2 l}(\mathbb{A})$ as in (2.14). The Fourier-Jacobi coefficient $\mathcal{F}_{\varphi_{2 r+l}}^{\psi}\left(\widetilde{E}_{1}\left(\cdot, \widetilde{\phi}_{\pi_{\psi}(\widetilde{\tau}) \otimes \tau}\right)\right)(g)$ of the residue $\widetilde{E}_{1}\left(\cdot, \widetilde{\phi}_{\pi_{\psi}(\widetilde{\tau}) \otimes \tau}\right)$ is given by the integral (as in (2.11))

$$
\mathcal{F}_{\varphi_{2 r+l}}^{\psi}\left(\widetilde{E}_{1}\left(\cdot, \widetilde{\phi}_{\pi_{\psi}(\widetilde{\tau}) \otimes \widetilde{\tau}}\right)\right)(g)=\int_{\left[V_{2 r+l}\right]} \widetilde{\theta}_{\varphi_{2 r+l}}^{\psi}\left(\ell_{2 r+l}(v) \widetilde{g}\right) \widetilde{E}_{1}\left(v \widetilde{g}, \widetilde{\phi}_{\pi_{\psi}(\widetilde{\tau}) \otimes \widetilde{\tau}}\right) \psi_{r-l}(v) d v .
$$


It is clear that the period integral in (4.2) may not converge and needs certain regularization to make it well defined. We follow the argument in [GJR01] to study this period.

4.1. Truncation of Eisenstein series. We recall a special case of the Arthur's truncation method and apply it to our study of the period integral as defined in (4.2). The notation we use is standard and can be found in [Jng98a or GJR01.

In this section we set $G=\mathrm{Sp}_{4 r+2 l}$ and $P=M U=P_{2 r, l}=M_{2 r, l} U_{2 r, l}$. We identify $\mathfrak{a}_{P}$ with $\mathbb{R}$ via $a \tilde{\alpha}_{2 r} \mapsto a$. Then a regular element in $\mathfrak{a}_{P}$ will correspond to a real number $c \in \mathbb{R}_{>1}$, where we denote by $\mathbb{R}_{>c}$ the set of all real numbers greater than $c$. We denote

$$
H(g):=\exp \left\langle 1, H_{P}(g)\right\rangle=|\operatorname{det} m(g)|
$$

for $g=u m(g) \mathrm{k} \in G(\mathbb{A})$ (the Iwasawa decomposition given in $\S 3.1)$. Let $\tau_{c}\left(c \in \mathbb{R}_{>1}\right)$ be the characteristic function of the subset $\mathbb{R}_{>c}$.

Following [A78] and A80, the truncation of the Eisenstein series $E(g, s, \phi)$ (where $\phi=\phi_{\pi_{\psi}(\widetilde{\tau}) \otimes \sigma}$ ) is defined as follows:

$$
\Lambda_{c} E(g, s, \phi)=E(g, s, \phi)-\sum_{\gamma \in P(k) \backslash G(k)} E_{P}(\gamma g, s, \phi) \tau_{c}(H(\gamma g)) .
$$

The constant term $E_{P}(g, s, \phi)$ of the Eisenstein series $E(g, s, \phi)$ along $P$ can be expressed as (see (3.5) for the definition)

$$
E_{P}(g, s, \phi)=\Phi(g, s, \phi)+\mathcal{M}(w, s)(\Phi(\cdot, s, \phi))(g)
$$

where $w=w_{2 r, l}$ and $\mathcal{M}(w, s)$ is the intertwining operator as described in $\S 3.1$. We remark that the summation in (4.5) has only finitely many terms and converges absolutely (Corollary 5.2 in [A78]). The truncated Eisenstein series can then be rewritten as

$$
\begin{aligned}
\Lambda_{c} E(g, s, \phi) & =\sum_{\gamma \in P(k) \backslash G(k)} \Phi(\gamma g, s, \phi)\left(1-\tau_{c}(H(\gamma g))\right) \\
& -\sum_{\gamma \in P(k) \backslash G(k)} \mathcal{M}(w, s)(\Phi(\cdot, s, \phi))(\gamma g) \tau_{c}(H(\gamma g)) \\
:= & \mathcal{E}_{1}(g)-\mathcal{E}_{2}(g) .
\end{aligned}
$$

Let $s_{0}$ be a positive real number. Consider the Laurent expansion of the Eisenstein series $E(g, s, \phi)$ at $s=s_{0}$ :

$$
E(g, s, \phi)=E_{s_{0}}(g, \phi)\left(s-s_{0}\right)^{e}+\text { higher terms }
$$

When $e=-1$, i.e., $s_{0}$ is a simple pole of $E(g, s, \phi)$, then $E_{s_{0}}(g, \phi)$ is the residue at $s=s_{0}$ of $E(g, s, \phi)$. In general we call $E_{s_{0}}(g, \phi)$ the leading term at $s=s_{0}$ of $E(g, s, \phi)$. In the following, we assume that $s_{0}>0$ is a simple pole of $E(g, s, \phi)$.

The truncation of the residue $E_{s_{0}}(g, \phi)$ is

$$
\begin{aligned}
\Lambda_{c} E_{s_{0}}(g, \phi) & =E_{s_{0}}(g, \phi)-\sum_{\gamma \in P(k) \backslash G(k)} \mathcal{M}(w, s)(\Phi(\cdot, s, \phi))_{s_{0}}(\gamma g) \tau_{c}(H(\gamma g)) \\
& :=E_{s_{0}}(g, \phi)-\mathcal{E}_{3}(g) .
\end{aligned}
$$

We consider the period integral $\mathcal{P}_{3 r, r-l}\left(E_{s_{0}}(\cdot, \phi), \widetilde{E}_{1}(\cdot, \widetilde{\phi}), \varphi_{2 r+l}\right)$ as defined in (4.2), but the residue $E_{\frac{1}{2}}(\cdot, \phi)$ is replaced by the residue $E_{s_{0}}(\cdot, \phi)$. Using (4.7), we 
obtain

$$
\begin{aligned}
& \mathcal{P}_{3 r, r-l}\left(E_{s_{0}}(\cdot, \phi), \widetilde{E}_{1}(\cdot, \widetilde{\phi}), \varphi_{2 r+l}\right) \\
= & \mathcal{P}_{3 r, r-l}\left(\mathcal{E}_{3}, \widetilde{E}_{1}(\cdot, \widetilde{\phi}), \varphi_{2 r+l}\right)+\mathcal{P}_{3 r, r-l}\left(\Lambda_{c} E_{s_{0}}(\cdot, \phi), \widetilde{E}_{1}(\cdot, \widetilde{\phi}), \varphi_{2 r+l}\right) .
\end{aligned}
$$

Since $\Lambda_{c} E(g, s, \phi)$ is rapidly decaying, the period integral

$$
\mathcal{P}_{3 r, r-l}\left(\Lambda_{c} E(g, s, \phi), \widetilde{E}_{1}(\cdot, \widetilde{\phi}), \varphi_{2 r+l}\right)
$$

defines a meromorphic function in $s$ with possible poles contained in the set of possible poles of the Eisenstein series $E(g, s, \phi)$, and hence in that of the global intertwining operator $\mathcal{M}(w, s)$. It follows that

$$
\operatorname{Res}_{s=s_{0}} \mathcal{P}_{3 r, r-l}\left(\Lambda_{c} E(g, s, \phi), \widetilde{E}_{1}(\cdot, \widetilde{\phi}), \varphi_{2 r+l}\right)=\mathcal{P}_{3 r, r-l}\left(\Lambda_{c} E_{s_{0}}(\cdot, \phi), \widetilde{E}_{1}(\cdot, \widetilde{\phi}), \varphi_{2 r+l}\right) \text {. }
$$

The following proposition will be proved in the next section.

Proposition 4.1. For $i=1,2$, the periods

$$
\mathcal{P}_{3 r, r-l}\left(\mathcal{E}_{i}, \widetilde{E}_{1}(\cdot, \widetilde{\phi}), \varphi_{2 r+l}\right)
$$

converge absolutely for $\operatorname{Re}(s)$ large and have meromorphic continuation to the whole complex plane.

By meromorphic continuation, we have

$$
\begin{aligned}
& \mathcal{P}_{3 r, r-l}\left(\Lambda_{c} E(g, s, \phi), \widetilde{E}_{1}(\cdot, \widetilde{\phi}), \varphi_{2 r+l}\right) \\
= & \mathcal{P}_{3 r, r-l}\left(\mathcal{E}_{1}, \widetilde{E}_{1}(\cdot, \widetilde{\phi}), \varphi_{2 r+l}\right)-\mathcal{P}_{3 r, r-l}\left(\mathcal{E}_{2}, \widetilde{E}_{1}(\cdot, \widetilde{\phi}), \varphi_{2 r+l}\right) .
\end{aligned}
$$

Hence we have

$$
\begin{aligned}
& \operatorname{Res}_{s=s_{0}} \mathcal{P}_{3 r, r-l}\left(\Lambda_{c} E(g, s, \phi), \widetilde{E}_{1}(\cdot, \widetilde{\phi}), \varphi_{2 r+l}\right) \\
= & \operatorname{Res}_{s=s_{0}} \mathcal{P}_{3 r, r-l}\left(\mathcal{E}_{1}, \widetilde{E}_{1}(\cdot, \widetilde{\phi}), \varphi_{2 r+l}\right)-\operatorname{Res}_{s=s_{0}} \mathcal{P}_{3 r, r-l}\left(\mathcal{E}_{2}, \widetilde{E}_{1}(\cdot, \widetilde{\phi}), \varphi_{2 r+l}\right) .
\end{aligned}
$$

From (4.6) and (4.7), one knows that

$$
\operatorname{Res}_{s=s_{0}} \mathcal{P}_{3 r, r-l}\left(\mathcal{E}_{2}, \widetilde{E}_{1}(\cdot, \widetilde{\phi}), \varphi_{2 r+l}\right)=\mathcal{P}_{3 r, r-l}\left(\mathcal{E}_{3}, \widetilde{E}_{1}(\cdot, \widetilde{\phi}), \varphi_{2 r+l}\right) .
$$

Therefore, we obtain

$$
\mathcal{P}_{3 r, r-l}\left(E_{s_{0}}(\cdot, \phi), \widetilde{E}_{1}(\cdot, \widetilde{\phi}), \varphi_{2 r+l}\right)=\operatorname{Res}_{s=s_{0}} \mathcal{P}_{3 r, r-l}\left(\mathcal{E}_{1}, \widetilde{E}_{1}(\cdot, \widetilde{\phi}), \varphi_{2 r+l}\right) .
$$

In other words, the study of the period $\mathcal{P}_{3 r, r-l}\left(E_{s_{0}}(\cdot, \phi), \widetilde{E}_{1}(\cdot, \widetilde{\phi}), \varphi_{2 r+l}\right)$ reduces to Proposition 4.1 and an explicit calculation of the integral

$$
\mathcal{P}_{3 r, r-l}\left(\mathcal{E}_{1}, \widetilde{E}_{1}(\cdot, \widetilde{\phi}), \varphi_{2 r+l}\right) .
$$

We will do this in the next section.

4.2. The proof of Proposition 4.1. First, the convergence for the real part of $s$ large of both integrals

$$
\mathcal{P}_{3 r, r-l}\left(\mathcal{E}_{1}, \widetilde{E}_{1}(\cdot, \widetilde{\phi}), \varphi_{2 r+l}\right) \text { and } \mathcal{P}_{3 r, r-l}\left(\mathcal{E}_{2}, \widetilde{E}_{1}(\cdot, \widetilde{\phi}), \varphi_{2 r+l}\right)
$$

follows as in the proof of Proposition 3.1 in GJR01. The meromorphic continuation of both periods and the possible location of poles will follow from the explicit calculation of the period integrals.

We shall calculate the period integral $\mathcal{P}_{3 r, r-l}\left(\mathcal{E}_{1}, \widetilde{E}_{1}(\cdot, \widetilde{\phi}), \varphi_{2 r+l}\right)$ for the real part of $s$ large, while the integral $\mathcal{P}_{3 r, r-l}\left(\mathcal{E}_{2}, \widetilde{E}_{1}(\cdot, \widetilde{\phi}), \varphi_{2 r+l}\right)$ can be calculated in a similar way to that in GJR01. 
Recall that $G=\operatorname{Sp}_{4 r+2 l}$ and $P=M U=P_{2 r, l}=M_{2 r, l} U_{2 r, l}$. Write $\phi=\phi_{\pi_{\psi}(\widetilde{\tau}) \otimes \sigma}$ and $\widetilde{\phi}=\widetilde{\phi}_{\pi_{\psi}(\widetilde{\tau}) \otimes \tilde{\tau}}$. Assume the real part of $s$ is large. The integral can be written first as

$$
\mathcal{P}_{3 r, r-l}\left(\mathcal{E}_{1}, \widetilde{E}_{1}(\cdot, \widetilde{\phi}), \varphi_{2 r+l}\right)=\int_{G(k) \backslash G(\mathbb{A})} \mathcal{E}_{1}(g) \mathcal{F}_{\varphi_{2 r+l}}^{\psi}\left(\widetilde{E}_{1}(\cdot, \widetilde{\phi})\right)(g) d g .
$$

From the discussion above, it is absolutely convergent when the real part of $s$ is large. By (4.6) we have

$$
\mathcal{E}_{1}(g)=\sum_{\gamma \in P(k) \backslash G(k)} \Phi(\gamma g, s, \phi)\left(1-\tau_{c}(H(\gamma g))\right) .
$$

Then (4.9) equals

$$
\int_{P(k) \backslash G(\mathbb{A})} \Phi(g, s, \phi)\left(1-\tau_{c}(H(g))\right) \mathcal{F}_{\varphi_{2 r+l}}^{\psi}\left(\widetilde{E}_{1}(\cdot, \widetilde{\phi})\right)(g) d g .
$$

Recall from (4.3) that

$$
\mathcal{F}_{\varphi_{2 r+l}}^{\psi}\left(\widetilde{E}_{1}(\cdot, \widetilde{\phi})\right)(g)=\int_{\left[V_{2 r+l}\right]} \widetilde{\theta}_{\varphi_{2 r+l}}^{\psi}\left(\ell_{2 r+l}(v) \widetilde{g}\right) \widetilde{E}_{1}(v \widetilde{g}, \widetilde{\phi}) \psi_{r-l}(v) d v,
$$

where $V_{2 r+l}=V_{3 r, 2 r+l}$ as defined in (2.1) with $p=3 r$ and $i=r-l$. From (2.7), we have

$$
\widetilde{\theta}_{\varphi_{2 r+l}}^{\psi}\left(\ell_{2 r+l}(v) \widetilde{g}\right)=\sum_{\xi \in k^{2 r+l}} \omega_{\psi}\left(\ell_{2 r+l}(v) \widetilde{g}\right) \varphi_{2 r+l}(\xi)
$$

where $\varphi_{2 r+l} \in \mathcal{S}\left(\mathbb{A}^{2 r+l}\right)$. We write $k^{2 r+l}=k^{2 r} \oplus k^{l}$ and $\xi=\left(\xi_{2 r}, \xi_{l}\right)$. Recall from (2.1) that elements of the unipotent subgroup $V_{2 r+l}$ can be expressed as

$$
\left\{v\left(n, p_{1}, p_{2}, p_{3}, z\right):=\left(\begin{array}{ccccc}
n & p_{1} & p_{2} & p_{3} & z \\
& I_{2 r} & 0 & 0 & p_{3}^{*} \\
& & I_{2 l} & 0 & p_{2}^{*} \\
& & & I_{2 r} & p_{1}^{*} \\
& & & & n^{*}
\end{array}\right) \in \mathrm{Sp}_{6 r}\right\}
$$

where $n \in N_{r-l}$, the standard maximal unipotent subgroup of $\mathrm{GL}_{r-l}$. The projection $\ell_{2 r+l}(v)$ of $v\left(n, p_{1}, p_{2}, p_{3}, z\right) \in V_{2 r+l}$ to the Heisenberg group $H_{4 r+2 l+1}$ (as defined in (2.2) with $p=3 r$ and $i=r-l$ ) can be expressed as (and identified with elements of $V_{2 r+l}$ )

$$
\ell_{2 r+l}\left(v\left(n, p_{1}, p_{2}, p_{3}, z\right)\right)=\left(\begin{array}{ccccccc}
I_{r-l-1} & & & & & & \\
& 1 & \bar{p}_{1} & \bar{p}_{2} & \bar{p}_{3} & \bar{z} & \\
& & I_{2 r} & & & * & \\
& & & I_{2 l} & & * & \\
& & & & I_{2 r} & * & \\
& & & & & 1 & \\
& & & & & & I_{r-l-1}
\end{array}\right) \in V_{2 r+l}
$$

where $\bar{p}_{i}$ is the last row of $p_{i}$, and $\bar{z}$ is the $(r-l, 1)$-th entry of $z$. For simplicity, we use the following notation for $\ell_{2 r+l}(v)$ :

$$
\ell_{2 r+l}\left(v\left(n, p_{1}, p_{2}, p_{3}, z\right)\right)=\ell\left(\bar{p}_{1}, \bar{p}_{2}, \bar{p}_{3}, \bar{z}\right) .
$$


To carry out our calculation, we write elements of $V_{2 r+l}$ as

$$
\left(\begin{array}{ccccc}
n & 0 & p_{2} & p_{3} & z \\
& I_{2 r} & 0 & 0 & p_{3}^{*} \\
& I_{2 l} & 0 & p_{2}^{*} \\
& & & I_{2 r} & 0 \\
& & & & n^{*}
\end{array}\right)\left(\begin{array}{ccccc}
I_{r-l} & p_{1} & & & \\
& I_{2 r} & & & \\
& & I_{2 l} & & \\
& & & I_{2 r} & p_{1}^{*} \\
& & & & I_{r-l}
\end{array}\right)=v\left(n, 0, p_{2}, p_{3}, z\right) v\left(p_{1}\right)
$$

where $v\left(p_{1}\right)=v\left(I_{r-l}, p_{1}, 0,0,0\right)$, and we write the elements $\ell\left(\bar{p}_{1}, \bar{p}_{2}, \bar{p}_{3}, \bar{z}\right)$ as

$$
\ell\left(\bar{p}_{1}, \bar{p}_{2}, \bar{p}_{3}, \bar{z}\right)=\ell\left(0, \bar{p}_{2}, \bar{p}_{3}, \bar{z}\right) \ell\left(\bar{p}_{1}, 0,0,0\right) .
$$

Then the Fourier-Jacobi coefficient $\mathcal{F}_{\varphi_{2 r+l}}^{\psi}\left(\widetilde{E}_{1}(\cdot, \widetilde{\phi})\right)(g)$ can be expressed as

$$
\begin{aligned}
\int_{\left[V_{2 r+l}\right]} & \sum_{\xi_{2 r} \in k^{2 r}, \xi_{l} \in k^{l}} \omega_{\psi}\left(\ell\left(0, \bar{p}_{2}, \bar{p}_{3}, \bar{z}\right) \ell\left(\bar{p}_{1}+\bar{p}_{1}\left(\xi_{2 r}\right), 0,0,0\right) \widetilde{g}\right) \varphi_{2 r+l}\left(\left(0, \xi_{l}\right)\right) \\
& \times \widetilde{E}_{1}\left(v\left(n, 0, p_{2}, p_{3}, z\right) v\left(p_{1}+p_{1}\left(\xi_{2 r}\right)\right) \widetilde{g}, \widetilde{\phi}\right) \psi_{r-l}(v) d v
\end{aligned}
$$

where $\bar{p}_{1}\left(\xi_{2 r}\right)$ is a matrix in $\operatorname{Mat}_{r-l, 2 r}(k)$ with the first $r-l-1$ rows zero and the last row equal to $\xi_{2 r}$. Let $V_{2 r+l}^{\prime}$ be the subgroup of $V_{2 r+l}$ consisting of elements $v\left(n, p_{1}, p_{2}, p_{3}, z\right)$ with the last row of $p_{1}$ zero. Collapsing the summation over $\xi_{2 r}$ with integration in (4.13), we obtain

$$
\begin{aligned}
\int_{V_{2 r+l}^{\prime}(k) \backslash V_{2 r+l}(\mathbb{A})} & \sum_{\xi_{l} \in k^{l}} \omega_{\psi}\left(\ell\left(0, \bar{p}_{2}, \bar{p}_{3}, \bar{z}\right) \ell\left(\bar{p}_{1}, 0,0,0\right) \widetilde{g}\right) \varphi_{2 r+l}\left(\left(0, \xi_{l}\right)\right) \\
& \times \widetilde{E}_{1}\left(v\left(n, 0, p_{2}, p_{3}, z\right) v\left(p_{1}\right) \widetilde{g}, \widetilde{\phi}\right) \psi_{r-l}(v) d v
\end{aligned}
$$

By the definition of the Weil representation $\omega_{\psi}$, we have

$$
\omega_{\psi}\left(\ell\left(0, \bar{p}_{2}, \bar{p}_{3}, \bar{z}\right) \widetilde{g}\right) \varphi_{2 r+l}\left(\left(0, \xi_{l}\right)\right)=\omega_{\psi}\left(\ell\left(0, \bar{p}_{2}, 0, \bar{z}\right) \widetilde{g}\right) \varphi_{2 r+l}\left(\left(0, \xi_{l}\right)\right) .
$$

Hence the Fourier-Jacobi coefficient $\mathcal{F}_{\varphi_{2 r+l}}^{\psi}\left(\widetilde{E}_{1}(\cdot, \widetilde{\phi})\right)(g)$ equals

$$
\begin{aligned}
\int_{V_{2 r+l}^{\prime}(k) \backslash V_{2 r+l}(\mathbb{A})} & \sum_{\xi_{l} \in k^{l}} \omega_{\psi}\left(\ell\left(0, \bar{p}_{2}, 0, \bar{z}\right) \ell\left(\bar{p}_{1}, 0,0,0\right) \widetilde{g}\right) \varphi_{2 r+l}\left(\left(0, \xi_{l}\right)\right) \\
& \times \widetilde{E}_{1}\left(v\left(n, 0, p_{2}, p_{3}, z\right) v\left(p_{1}\right) \widetilde{g}, \widetilde{\phi}\right) \psi_{r-l}(v) d v .
\end{aligned}
$$

It follows that integral (4.10) equals

$$
\begin{aligned}
\int_{P(k) \backslash G(\mathbb{A})} \Phi^{c}(g, s, \phi) & \int_{V_{2 r+l}^{\prime}(k) \backslash V_{2 r+l}(\mathbb{A})} \sum_{\xi_{l} \in k^{l}} \omega_{\psi}\left(\ell\left(\bar{p}_{2}, \bar{z}\right) \ell\left(\bar{p}_{1}\right) \widetilde{g}\right) \varphi_{2 r+l}\left(\left(0, \xi_{l}\right)\right) \\
& \times \widetilde{E}_{1}\left(v\left(n, 0, p_{2}, p_{3}, z\right) v\left(p_{1}\right) \widetilde{g}, \widetilde{\phi}\right) \psi_{r-l}(v) d v d g
\end{aligned}
$$

where $\Phi^{c}(g, s, \phi)=\Phi(g, s, \phi)\left(1-\tau_{c}(H(g))\right)$, and $\ell\left(\bar{p}_{2}, \bar{z}\right)=\ell\left(0, \bar{p}_{2}, 0, \bar{z}\right)$ and $\ell\left(\bar{p}_{1}\right)=$ $\ell\left(\bar{p}_{1}, 0,0,0\right)$.

It is easy to check from the definition of the Weil representation $\omega_{\psi}$ (Chapter 2 in [MVW87]) that as a function of $\widetilde{g}$ the function

$$
\sum_{\xi_{l} \in k^{l}} \omega_{\psi}\left(\ell\left(\bar{p}_{2}, \bar{z}\right) \ell\left(\bar{p}_{1}\right) \widetilde{g}\right) \varphi_{2 r+l}\left(\left(0, \xi_{l}\right)\right)
$$


is left $U_{2 r, l}(\mathbb{A})$-invariant. Recall that the unipotent radical $U=U_{2 r, l}$ of $P_{2 r, l}$ has the form

$$
\left\{u\left(y, z^{\prime}\right):=\left(\begin{array}{ccc}
I_{2 r} & y & z^{\prime} \\
& I_{2 l} & y^{*} \\
& & I_{2 r}
\end{array}\right) \in \operatorname{Sp}_{4 r+2 l}\right\} .
$$

In the calculation, $\mathrm{Sp}_{4 r+2 l}$ has been embedded into the 'middle' of $\mathrm{Sp}_{6 r}$. By factoring the integration over $U_{2 r, l}(k) \backslash U_{2 r, l}(\mathbb{A})$ from $P(k) \backslash G(\mathbb{A})$, we obtain that integral (4.16) equals

$$
\int \Phi^{c}(g, s, \phi) \int_{\left[U_{2 r, l}\right]} \int_{V_{2 r+l}^{\prime}(k) \backslash V_{2 r+l}(\mathbb{A})} \sum_{\xi_{l} \in k^{l}} \omega_{\psi}\left(\ell\left(\bar{p}_{2}, \bar{z}\right) \ell\left(\bar{p}_{1}\right) \widetilde{g}\right) \varphi_{2 r+l}\left(\left(0, \xi_{l}\right)\right)
$$

$$
\times \widetilde{E}_{1}\left(v\left(n, 0, p_{2}, p_{3}, z\right) v\left(p_{1}\right) u \widetilde{g}, \widetilde{\phi}\right) \psi_{r-l}(v) d v d p_{1} d u d g,
$$

where the integration in variable $g$ is over $M(k) U(\mathbb{A}) \backslash G(\mathbb{A})$.

The integrations over $\left[U_{2 r, l}\right]$ and $V_{2 r+l}^{\prime}(k) \backslash V_{2 r+l}(\mathbb{A})$ can be rewritten as follows. We can rewrite $v\left(n, 0, p_{2}, p_{3}, z\right) v\left(p_{1}\right) u$ as

$$
\left(\begin{array}{ccccc}
n & p_{1} & p_{2} & p_{3} & z \\
& I_{2 r} & y & z^{\prime} & p_{3}^{*} \\
& & I_{2 l} & y^{*} & p_{2}^{*} \\
& & & I_{2 r} & p_{1}^{*} \\
& & & & n^{*}
\end{array}\right)=\left(\begin{array}{ccccc}
n & 0 & p_{2} & p_{3} & z \\
& I_{2 r} & y & z^{\prime} & p_{3}^{*} \\
& & I_{2 l} & y^{*} & p_{2}^{*} \\
& & & I_{2 r} & 0 \\
& & & & n^{*}
\end{array}\right) v\left(p_{1}\right) .
$$

Denote by $V^{0}$ the group consisting of elements

$$
v^{0}=\left(\begin{array}{ccccc}
n & 0 & p_{2} & p_{3} & z \\
& I_{2 r} & y & z^{\prime} & p_{3}^{*} \\
& & I_{2 l} & y^{*} & p_{2}^{*} \\
& & & I_{2 r} & 0 \\
& & & & n^{*}
\end{array}\right)
$$

Note that the element of $U_{2 r, l}$ sits in the middle of $v^{0}$. Hence in (4.18) the inner integrations over $\left[U_{2 r, l}\right]$ and $V_{2 r+l}^{\prime}(k) \backslash V_{2 r+l}(\mathbb{A})$ can be rewrite as

$$
\iint_{\left[V^{0}\right]} \sum_{\xi_{l} \in k^{l}} \omega_{\psi}\left(\ell\left(\bar{p}_{2}, \bar{z}\right) \ell\left(\bar{p}_{1}\right) \widetilde{g}\right) \varphi_{2 r+l}\left(\left(0, \xi_{l}\right)\right) \widetilde{E}_{1}\left(v^{0} v\left(p_{1}\right) \widetilde{g}, \widetilde{\phi}\right) \psi_{r-l}\left(v^{0}\right) d v^{0} d p_{1},
$$

where the integration $\int d p_{1}$ along the variable $p_{1}$ is in $\mathrm{Mat}_{r-l, 2 r}$ with the first $r-l-1$ rows in $\operatorname{Mat}_{r-l-1,2 r}(k) \backslash \operatorname{Mat}_{r-l-1,2 r}(\mathbb{A})$ and the last row in $\mathbb{A}^{2 r}$.

To calculate the last integral, we define the Weyl element $w$ of $S p_{6 n}$ as

$$
w=\left(\begin{array}{ccccc} 
& I_{2 n} & & & \\
I_{n-k} & & & & \\
& & I_{2 k} & & \\
& & & I_{2 n} & I_{n-k}
\end{array}\right) .
$$

We conjugate in $\widetilde{E}_{1}\left(v^{0} v\left(p_{1}\right) \widetilde{g}, \widetilde{\phi}\right)$ by $w$ from left to right and obtain that the last integral equals

$$
\iint_{\left[V^{(0)}\right]} \sum_{\xi_{l} \in k^{l}} \omega_{\psi}\left(\ell\left(\bar{p}_{2}, \bar{z}\right) \ell\left(\bar{p}_{1}\right) \widetilde{g}\right) \varphi_{2 r+l}\left(\left(0, \xi_{l}\right)\right) \widetilde{E}_{1}\left(v^{(0)} v^{-}\left(p_{1}\right) w \widetilde{g}, \widetilde{\phi}\right) \psi_{r-l}\left(v^{(0)}\right) d v^{(0)} d p_{1} .
$$


Note that the integration $\int d p_{1}$ along the variable $p_{1}$ is the same as the above. The unipotent subgroup $V^{(0)}$ consists of elements of the type

$$
v^{(0)}=\left(\begin{array}{ccccc}
I_{2 r} & & y & p_{3}^{*} & z^{\prime} \\
& n & p_{2} & z & p_{3} \\
& & I_{2 l} & p_{2}^{*} & y^{*} \\
& & & n^{*} & \\
& & & & I_{2 r}
\end{array}\right)
$$

and

$$
v^{-}\left(p_{1}\right):=\left(\begin{array}{ccccc}
I_{2 r} & & & & \\
p_{1} & I_{r-l} & & & \\
& & I_{2 l} & & \\
& & & I_{r-l} & \\
& & & p_{1}^{*} & I_{2 r}
\end{array}\right) .
$$

To continue the calculation of (4.19), we formulate a general lemma below. Let $\phi$ be a smooth automorphic form on $\mathrm{GL}_{i+j}(\mathbb{A})(i, j \geq 2)$. We consider integrals of the type

$$
\int_{p} \int_{n} \phi\left(\left(\begin{array}{ll}
I_{i} & \\
& n
\end{array}\right)\left(\begin{array}{ll}
I_{i} & \\
p & I_{j}
\end{array}\right)\right) \psi_{j}(n) d n d p
$$

where the integration in variable $n$ is over $N_{j}(k) \backslash N_{j}(\mathbb{A}), \psi_{j}(n)$ is the generic character of $N_{j}$ as defined in (2.3), and the integration in variable $p$ is over the quotient $\operatorname{Mat}_{j-1, i}(k) \backslash \operatorname{Mat}_{j, i}(\mathbb{A})$; i.e., the last row of $p$ is integrated over the $\mathbb{A}$-points.

Lemma 4.2. Let $\phi$ be a smooth automorphic form on $\mathrm{GL}_{i+j}(\mathbb{A})(i, j \geq 2)$. Then integral (4.20) is equal to

$$
\int_{p} \int_{n} \int_{q} \phi\left(\left(\begin{array}{cc}
I_{i} & q \\
& n
\end{array}\right)\left(\begin{array}{cc}
I_{i} & \\
p & I_{j}
\end{array}\right)\right) d q \psi_{j}(n) d n d p
$$

where the integration in variable $q$ is over $\operatorname{Mat}_{i, j}(k) \backslash \operatorname{Mat}_{i, j}(\mathbb{A})$ with the first column of $q$ being zero, the integration in variable $p$ is over $\operatorname{Mat}_{j, i}(\mathbb{A})$, and the integration in variable $n$ is the same as in (4.20).

Proof. We define the following unipotent subgroups of $\mathrm{GL}_{i+j}$. For $1 \leq m \leq j$ define ( $C$ means 'column')

$$
C_{m}=\left\{\left(\begin{array}{cc}
I_{i} & q \\
& I_{j}
\end{array}\right): q \in \mathrm{Mat}_{i, j}, \quad q_{\mu, \nu}=0, \quad \nu \neq m\right\} .
$$

In other words, $C_{m}$ is the group of all matrices as above such that all columns in $q$ are zero except the $m$-th column. Note that $C_{m}(k)$ is isomorphic to $k^{i}$. For $1 \leq m \leq j-1$ we also define ( $R$ means 'row')

$$
R_{m}=\left\{\left(\begin{array}{cc}
I_{i} & \\
p & I_{j}
\end{array}\right): p \in \operatorname{Mat}_{j, i}, \quad p_{\mu, \nu}=0, \quad \mu \neq m\right\} .
$$

Thus $R_{m}$ consists of all matrices as above such that all rows in $p$, except the $m$-th row, are zero. 
We consider first the Fourier expansion of the automorphic form $\phi$ along the unipotent subgroup $C_{j}$ :

$$
\phi(g)=\sum_{\xi \in k^{i}} \int_{C_{j}(k) \backslash C_{j}(\mathbb{A})} \phi\left(\left(\begin{array}{cc}
I_{i} & q \\
& I_{j}
\end{array}\right) g\right) \psi(\xi \cdot q) d q .
$$

Plugging this into (4.20), we have

$$
\int_{p} \int_{n} \sum_{\xi \in k^{i}} \int_{C_{j}(k) \backslash C_{j}(\mathbb{A})} \phi\left(\left(\begin{array}{ll}
I_{i} & q \\
& n
\end{array}\right)\left(\begin{array}{ll}
I_{i} & \\
p & I_{j}
\end{array}\right)\right) \psi(\xi \cdot q) d q \psi_{j}(n) d n d p .
$$

Note that $\left(\begin{array}{cc}I_{i} & q \\ & I_{j}\end{array}\right)\left(\begin{array}{cc}I_{i} & \\ & n\end{array}\right)=\left(\begin{array}{cc}I_{i} & q^{\prime} \\ & n\end{array}\right)$. Since $R_{j-1}(k)$ is isomorphic to $k^{i}$, we identify $\xi \in k^{i}$ with the element

$$
\left(\begin{array}{cc}
I_{i} & \\
\xi & I_{j}
\end{array}\right) \in R_{j-1}(k)
$$

Since $\phi$ is automorphic, we have

$$
\phi\left(\left(\begin{array}{cc}
I_{i} & \\
\xi & I_{j}
\end{array}\right) g\right)=\phi(g)
$$

It is easy to show that

$$
\left(\begin{array}{cc}
I_{i} & 0 \\
\xi & I_{j}
\end{array}\right)\left(\begin{array}{cc}
I_{i} & q \\
0 & n
\end{array}\right)\left(\begin{array}{cc}
I_{i} & 0 \\
p & I_{j}
\end{array}\right)=\left(\begin{array}{cc}
I_{i} & q \\
0 & n+\xi \cdot q
\end{array}\right)\left(\begin{array}{cc}
I_{i} & 0 \\
p+n^{-1} \xi & I_{j}
\end{array}\right)
$$

where $\xi \cdot q$ is a $j \times j$-matrix with all entries zero except the $(j-1, j)$-th entry, which is

$$
(\xi \cdot q)_{j-1, j}=\sum_{l=1}^{i} \xi_{l} q_{l, j}
$$

Since $n^{-1}$ is upper trianglar and $\xi$ is in $R_{j-1}$, we may write

$$
n^{-1} \xi=p_{2}+\xi
$$

where $p_{2}$ is in Mat $j, i$ with the last two rows zero.

Applying the above calculation to (4.21) and changing variables, we obtain

$$
\int_{p} \int_{n} \int_{C_{j}(k) \backslash C_{j}(\mathbb{A})} \phi\left(\left(\begin{array}{cc}
I_{i} & q \\
& n
\end{array}\right)\left(\begin{array}{cc}
I_{i} & \\
p & I_{j}
\end{array}\right)\right) d q \psi_{j}(n) d n d p
$$

where the integration of $p$ is on $\operatorname{Mat}_{j-2, i}(k) \backslash \operatorname{Mat}_{j, i}(\mathbb{A})$; i.e., the last two rows of $p$ are integrated over the $\mathbb{A}$-points.

We apply the same argument above with a sequence of pairs

$$
\left(C_{j-1}, R_{j-2}\right),\left(C_{j-2}, R_{j-3}\right), \cdots,\left(C_{2}, R_{1}\right)
$$

to integral (4.22) and complete the proof of this lemma. 
In order to apply Lemma 4.2 to the calculation of integral (4.19), we define the following unipotent subgroups of $\mathrm{Sp}_{6 r}$. For $1 \leq m \leq r-l$ define

$$
\mathcal{C}_{m}=\left\{\left(\begin{array}{ccccc}
I_{2 r} & q & & & \\
& I_{r-l} & & & \\
& & I_{2 l} & & \\
& & & I_{r-l} & q^{*} \\
& & & & I_{2 r}
\end{array}\right): q \in \operatorname{Mat}_{2 r, r-l}, q_{i, j}=0, \quad j \neq m\right\} .
$$

It is clear that $\mathcal{C}_{m}$ is an embedding of $C_{m}$ into $\operatorname{Sp}_{6 r}$ (with $i=2 r$ and $j=r-l$ ) and that it is the group of all matrices as above such that all columns in $q$ are zero except the $m$-th column. For $1 \leq m \leq r-l-1$ we also define

$$
\mathcal{R}_{m}=\left\{\left(\begin{array}{ccccc}
I_{2 r} & & & & \\
p & I_{r-l} & & & \\
& & I_{2 l} & & \\
& & & I_{r-l} & \\
& & & p^{*} & I_{2 r}
\end{array}\right): p \in \text { Mat }_{r-l, 2 r}, \quad p_{i, j}=0, \quad i \neq m\right\} .
$$

Thus $\mathcal{R}_{m}$ consists of all matrices as above such that all rows in $p$, except the $m$-th row, are zero, and it is an embedding of $R_{m}$ in $\mathrm{Sp}_{6 r}$. We want to apply Lemma 4.2 to integral (4.19). In this case we have to apply the argument to the series of pairs

$$
\left(\mathcal{C}_{r-l}, \mathcal{R}_{r-l-1}\right),\left(\mathcal{C}_{r-l-1}, \mathcal{R}_{r-l-2}\right), \cdots,\left(\mathcal{C}_{2}, \mathcal{R}_{1}\right) .
$$

Because in (4.19) the variable $\ell\left(p_{1}\right)$ is related to $\mathcal{R}_{r-l}$ and commutes with the subgroups $\mathcal{R}_{r-l-1}, \mathcal{R}_{r-l-2}, \cdots, \mathcal{R}_{1}$, we can apply the argument of the proof of Lemma 4.2 essentially to the automorphic form $\widetilde{E}_{1}\left(v^{(1)} v^{-}\left(p_{1}\right) w \widetilde{g}, \widetilde{\phi}\right)$. By Lemma 4.2 , integral (4.19) equals

$$
\iint_{\left[V^{(1)}\right]} \sum_{\xi_{l} \in k^{l}} \omega_{\psi}\left(\ell\left(\bar{p}_{2}, \bar{z}\right) \ell\left(\bar{p}_{1}\right) \widetilde{g}\right) \varphi_{2 r+l}\left(\left(0, \xi_{l}\right)\right) \widetilde{E}_{1}\left(v^{(1)} v^{-}\left(p_{1}\right) w \widetilde{g}, \widetilde{\phi}\right) \psi_{r-l}\left(v^{(1)}\right) d v^{(1)} d p_{1},
$$

where the integration in variable $p_{1}$ is over $\operatorname{Mat}_{r-l, 2 r}(\mathbb{A})$. The unipotent subgroup $V^{(1)}$ consists of elements of the type

$$
v^{(1)}=\left(\begin{array}{ccccc}
I_{2 r} & q & y & p_{3}^{*} & z^{\prime} \\
& n & p_{2} & z & p_{3} \\
& & I_{2 l} & p_{2}^{*} & y^{*} \\
& & & n^{*} & q^{*} \\
& & & & I_{2 r}
\end{array}\right)
$$

where $q$ is in Mat $_{2 r, r-l}$ with the first column zero.

Finally we consider the Fourier expansion of the residue $\widetilde{E}_{1}(\widetilde{g}, \widetilde{\phi})$ along the unipotent group $\mathcal{C}_{1}(k) \backslash \mathcal{C}_{1}(\mathbb{A})$. The group of characters of $\mathcal{C}_{1}(k) \backslash \mathcal{C}_{1}(\mathbb{A})$ is isomorphic to $k^{2 r}$ and the group $G L_{2 r}(k)$ acts on $k^{2 r}$ with two orbits. Hence the Fourier expansion of $\widetilde{E}_{1}(\widetilde{g}, \widetilde{\phi})$ along the unipotent group $\mathcal{C}_{1}(k) \backslash \mathcal{C}_{1}(\mathbb{A})$ is

$$
\begin{aligned}
\widetilde{E}_{1}(\widetilde{g}, \widetilde{\phi})= & \int_{\mathcal{C}_{1}(k) \backslash \mathcal{C}_{1}(\mathbb{A})} \widetilde{E}_{1}(v(q) \widetilde{g}, \widetilde{\phi}) d v(q) \\
& +\sum_{\gamma \in P^{1}(k) \backslash \mathrm{GL}_{2 r}(k)} \int_{\mathcal{C}_{1}(k) \backslash \mathcal{C}_{1}(\mathbb{A})} \widetilde{E}_{1}\left(v(q) m\left(\gamma, I_{2 r}\right) \widetilde{g}, \widetilde{\phi}\right) \psi\left(q_{2 r, 1}\right) d v(q),
\end{aligned}
$$

where $m\left(\gamma, I_{2 r}\right) \in \mathrm{GL}_{2 r}(k) \times \operatorname{Sp}_{2 r}(k)$ and $q_{2 r, 1}$ is the $(2 r, 1)$-th entry of $q$ in $v(q) \in \mathcal{C}_{1}$. 
Applying (4.24) to (4.23), integral (4.23) equals a sum of integral $I_{0}$ and integral $I_{1}$, defined as follows.

Integral $I_{0}$ is obtained from the constant term in (4.24), that is,

$$
\int_{\mathcal{C}_{1}(k) \backslash \mathcal{C}_{1}(\mathbb{A})} \widetilde{E}_{1}(v(q) \widetilde{g}, \widetilde{\phi}) d v(q) .
$$

The product of the unipotent subgroups $V^{(1)}$ and $\mathcal{C}_{1}$ can be written as a product $U_{2 r, r} \cdot V_{r, l}$; i.e.,

$$
\left(\begin{array}{ccccc}
I_{2 r} & q & y & p_{3}^{*} & z^{\prime} \\
& n & p_{2} & z & p_{3} \\
& I_{2 l} & p_{2}^{*} & y^{*} \\
& & & n^{*} & q^{*}
\end{array}\right)=\left(\begin{array}{cccccc}
I_{2 r} & q & y & p_{3}^{*} & z^{\prime} \\
& I_{r-l} & & & p_{3} \\
& & I_{2 l} & & y^{*} \\
& & & I_{r-l} & q^{*} \\
& & & & & \\
& & & & I_{2 r}
\end{array}\right)\left(\begin{array}{ccccc}
I_{2 r} & & & & \\
& n & p_{2} & z & \\
& & I_{2 l} & p_{2}^{*} & \\
& & n^{*} & \\
& & & & I_{2 r}
\end{array}\right) .
$$

Here $V_{r, l} \subset \mathrm{Sp}_{2 r}$ is as defined in (2.1) with $p=r$ and $i=r-l$ and is embedded in $\mathrm{Sp}_{6 r}$ as above. Hence integral $I_{0}$ equals

$\iint_{\left[V_{r, l}\right]} \sum_{\xi_{l} \in k^{l}} \omega_{\psi}\left(\ell\left(\bar{p}_{2}, z\right) \ell\left(\bar{p}_{1}\right) \widetilde{g}\right) \varphi_{2 r+l}\left(\left(0, \xi_{l}\right)\right) \widetilde{E}_{1, P_{2 r, r}}\left(v v^{-}\left(p_{1}\right) w \widetilde{g}, \widetilde{\phi}\right) \psi_{r-l}(v) d v d p_{1} d g$,

where the integration in variable $p_{1}$ is over $\operatorname{Mat}_{r-l, 2 r}(\mathbb{A})$, and $\widetilde{E}_{1, P_{2 r, r}}(\widetilde{g}, \widetilde{\phi})$ is the constant term along the maximal parabolic subgroup $P_{2 r, r}$.

Integral $I_{1}$ is obtained from the sum of the nontrivial Fourier coefficients in (4.24), that is,

$$
\sum_{\gamma \in P^{1}(k) \backslash \mathrm{GL}_{2 r}(k)} \int_{\mathcal{C}_{1}(k) \backslash \mathcal{C}_{1}(\mathbb{A})} \widetilde{E}_{1}\left(v(q) m\left(\gamma, I_{4 r}\right) \widetilde{g}, \widetilde{\phi}\right) \psi\left(q_{2 r, 1}\right) d v(q) .
$$

We now show that integral $I_{1}$ is zero. Since $I_{1}$ can be written as a sum of integrals parameterized by $\gamma \in P^{1}(k) \backslash \mathrm{GL}_{2 r}(k)$, we will show that each of these summands is zero. As in (4.25) (or in the case of $I_{0}$ ) the integration over $\left[V^{(1)}\right]$ combined with that over $\left[\mathcal{C}_{1}\right]$ is the same as the integration over the product of $\left[U_{2 r, r}\right]$ and $\left[V_{r, l}\right]$. The elements of $U_{2 r, r}$ are of the form

$$
u\left(q, y, p_{3}, z^{\prime}\right)=\left(\begin{array}{ccccc}
I_{2 r} & q & y & p_{3}^{*} & z^{\prime} \\
& I_{r-l} & & & p_{3} \\
& & I_{2 l} & & y^{*} \\
& & & I_{r-l} & q^{*} \\
& & & & I_{2 r}
\end{array}\right) \in \mathrm{Sp}_{6 r}
$$

and the elements of $V_{r, l}$ are of the form

$$
v\left(n, p_{2}, z\right)=\left(\begin{array}{ccccc}
I_{2 r} & & & & \\
& n & p_{2} & z & \\
& & I_{2 l} & p_{2}^{*} & \\
& & & n^{*} & \\
& & & & I_{2 r}
\end{array}\right) \in \operatorname{Sp}_{6 r} .
$$

The difference between $I_{0}$ and $I_{1}$ is that in $I_{0}$ it produces the constant term of $\widetilde{E}_{1}(\widetilde{g}, \widetilde{\phi})$ with respect to $U_{2 r, r}$, but in $I_{1}$ it produces the nontrivial Fourier coefficient of $\widetilde{E}_{1}(\widetilde{g}, \widetilde{\phi})$ with respect to $U_{2 r, r}$ and the nontrivial character $\psi\left(q_{2 r, 1}\right)$.

The point is that this nontrivial Fourier coefficient of the residue $\widetilde{E}_{1}(\widetilde{g}, \widetilde{\phi})$ with respect to $U_{2 r, r}$ and the nontrivial character $\psi\left(q_{2 r, 1}\right)$ combined with nontrivial 
Fourier coefficient of $\widetilde{E}_{1}(\widetilde{g}, \widetilde{\phi})$ with respect to $V_{r, l}$ and the nontrivial character $\psi_{r-l}\left(v\left(n, p_{2}, z\right)\right)$ produces a Fourier-Jacobi coefficient of the residue $\widetilde{E}_{1}(\widetilde{g}, \widetilde{\phi})$ associated to the unipotent orbit with symplectic partition $\left((6 r-2 l) 1^{2 l}\right)$. Hence the nonvanishing of each summand of $I_{1}$ implies that the residue $\widetilde{E}_{1}(\widetilde{g}, \widetilde{\phi})$ has a nonzero Fourier-Jacobi coefficient attached to the unipotent orbit with symplectic partition $\left((6 r-2 l) 1^{2 l}\right)$. On the other hand, by Proposition 1 of [GRS] and since $r \geq l$, the residue $\widetilde{E}_{1}(\widetilde{g}, \widetilde{\phi})$ cannot have any nonzero Fourier-Jacobi coefficient attached to such an orbit. This proves that each summand in $I_{1}$ is identically zero, and so is $I_{1}$.

To carry out the above argument, we consider first the Fourier coefficient

$$
\int_{\left[U_{2 r, r}\right]} \widetilde{E}_{1}\left(u\left(q, y, p_{3}, z^{\prime}\right) \widetilde{g}, \widetilde{\phi}\right) \psi\left(q_{2 r, 1}\right) d u .
$$

In (4.26), we further consider Fourier expansion (as a function of $\widetilde{g}$ ) along the maximal unipotent subgroup $N_{2 r}$ of $\mathrm{GL}_{2 r}$. Recall that the parabolic subgroup is

$$
P_{2 r, r}=\left(\mathrm{GL}_{2 r} \times \mathrm{Sp}_{2 r}\right) U_{2 r, r} .
$$

Since the residue $\widetilde{E}_{1}(\widetilde{g}, \widetilde{\phi})$ has the cuspidal support

$$
\left(\mathrm{GL}_{2 r} \times{\widetilde{\mathrm{Sp}_{2 r}}}, \pi_{\psi}(\widetilde{\tau}) \otimes \widetilde{\tau}\right)
$$

the constant terms along the subgroups of $N_{2 r}$ of integral (4.26) (as a function in $\widetilde{g}$ ) are zero. We end up with the Fourier expansion similar to the well-known Whittaker-Fourier expansion for a cuspidal automorphic form of $\mathrm{GL}_{2 r}(\mathbb{A})$ (PS71 and [Shl74]); i.e., (4.26) equals

$$
\sum_{\delta \in N_{2 r}(k) \backslash \mathrm{GL}_{2 r}(k)} \int_{\left[U_{2 r, r]}\right.} \int_{\left[N_{2 r}\right]} \widetilde{E}_{1}\left(n u\left(q, y, p_{3}, z^{\prime}\right) \widetilde{g}, \widetilde{\phi}\right) \psi_{2 r}^{\delta}(n) d n \psi\left(q_{2 r, 1}\right) d u,
$$

where $\psi_{2 r}^{\delta}(n)$ is the generic character corresponding to $\delta$. For instance, when $\delta=$ $I_{2 r}$, the identity element, the character $\psi_{2 r}^{\delta}(n)=\psi_{2 r}(n)$ is the generic character as defined in (2.3) (with $i$ replaced by $2 r$ ). Combining (4.27) with the unipotent integration along $V_{r, l}$ in integral $I_{1}$ (as in $I_{0}$ in (4.25)), we obtain as an inner integration in each summand of $I_{1}$ the integral

$$
\begin{aligned}
\int_{\left[U_{2 r, r}\right]} \int_{\left[N_{2 r}\right]} \int_{\left[V_{r, l]}\right]} & \sum_{\xi_{l} \in k^{l}} \omega_{\psi}\left(\ell\left(\bar{p}_{2}, \bar{z}\right) \ell\left(\bar{p}_{1}\right) \widetilde{g}\right) \varphi_{2 r+l}\left(\left(0, \xi_{l}\right)\right) \\
& \times \widetilde{E}_{1}\left(v n u\left(q, y, p_{3}, z^{\prime}\right) \widetilde{g}, \widetilde{\phi}\right) \psi_{r-l}(v) d v \psi_{2 r}^{\delta}(n) d n \psi\left(q_{2 r, 1}\right) d u .
\end{aligned}
$$

This integral is parameterized by $\delta \in P^{1}(k) \backslash \mathrm{GL}_{2 r}(k)$. Note that elements $\delta$ also stabilizes the $k$-rational orbit of the character $\psi\left(q_{2 r, 1}\right)$ in (4.24). For automorphic forms, the nonvanishing of Fourier coefficients depends only on a $k$-rational orbit of the characters. It follows that in order to prove the vanishing of each summand in $I_{1}$, it is enough to show the vanishing of (4.28) with $\delta$ being the identity.

Note that the product of unipotent subgroups $N_{2 r}, U_{2 r, r}$, and $V_{r, l}$ is $V_{3 r, l}$ (see (2.1) for the definition with $p=3 r$ and $i=3 r-l$ ) of $\mathrm{Sp}_{6 r}$ and the product of characters $\psi_{r-l}(v), \psi_{2 r}(n)$, and $\psi\left(q_{2 r, 1}\right)$ is the generic character of $N_{3 r-l}$ (see $(2.3))$. Hence integral (4.28) is the Fourier-Jacobi coefficient of the residue $\widetilde{E}_{1}(\widetilde{g}, \widetilde{\phi})$ attached to the unipotent orbit with partition $\left((6 r-2 l) 1^{2 l}\right)$. They are all zero by Proposition 1 in GRS. This proves that $I_{1}$ is identically zero. 
Therefore, from the sum of $I_{0}$ and $I_{1}$, there remains one integral in (4.25), which may be nonzero. In other words, from (4.18), (4.19), and (4.25), we obtain the following proposition.

Proposition 4.3. The period $\mathcal{P}_{3 r, r-l}\left(\mathcal{E}_{1}, \widetilde{E}_{1}(\cdot, \widetilde{\phi}), \varphi_{2 r+l}\right)$ defined in (4.9) equals integral (4.25), that is,

$$
\begin{aligned}
\int \Phi^{c}(g, s, \phi) & \int_{\operatorname{Mat}_{r-l, 2 r}(\mathbb{A})} \int_{\left[V_{r, l]} \sum_{\xi_{l} \in k^{l}} \omega_{\psi}\left(\ell\left(\bar{p}_{2}, \bar{z}\right) \ell\left(\bar{p}_{1}\right) \widetilde{g}\right) \varphi_{2 r+l}\left(\left(0, \xi_{l}\right)\right)\right.} \\
& \times \widetilde{E}_{1, P_{2 r, r}}\left(v v^{-}\left(p_{1}\right) w \widetilde{g}, \widetilde{\phi}\right) \psi_{r-l}(v) d v d p_{1} d g
\end{aligned}
$$

where the integration in variable $g$ is over $M(k) U(\mathbb{A}) \backslash G(\mathbb{A})$, and $\widetilde{E}_{1, P_{2 r, r}}(\widetilde{g}, \widetilde{\phi})$ is the constant term of the residue $\widetilde{E}_{1}(\widetilde{g}, \widetilde{\phi})$ along the maximal parabolic subgroup $P_{2 r, r}$.

In the following we shall simplify the integral in Proposition 4.3. First we write

$$
\widetilde{\theta}_{\varphi_{2 r+l}, l}\left(\ell\left(\bar{p}_{2}, \bar{z}\right) \ell\left(\bar{p}_{1}\right) \widetilde{g}\right):=\sum_{\xi_{l} \in k^{l}} \omega_{\psi}\left(\ell\left(\bar{p}_{2}, \bar{z}\right) \ell\left(\bar{p}_{1}\right) \widetilde{g}\right) \varphi_{2 r+l}\left(\left(0, \xi_{l}\right)\right)
$$

The constant term $\widetilde{E}_{1, P_{2 r, r}}(\widetilde{g}, \widetilde{\phi})$ of the residue $\widetilde{E}_{1}(\widetilde{g}, \widetilde{\phi})$ along the standard maximal parabolic subgroup $\widetilde{P}_{2 r, r}$ equals $\widetilde{\mathcal{M}}_{1}(\widetilde{\Phi})(\widetilde{g})$, which is the residue at $s=1$ of the intertwining operator $\widetilde{\mathcal{M}}\left(w_{2 r, r}, s\right)(\widetilde{\Phi})(\widetilde{g})$ defined in $\S 3.2$. By the definition in $\S 3.2$, $\widetilde{\mathcal{M}}\left(w_{2 r, r}, s\right)$ maps sections

$$
\widetilde{\Phi}=\widetilde{\Phi}\left(\widetilde{g}, s, \widetilde{\phi}_{\pi_{\psi}(\widetilde{\tau}) \otimes \widetilde{\tau}}\right) \in \widetilde{I}\left(s, \pi_{\psi}(\widetilde{\tau}) \otimes \widetilde{\tau}\right)
$$

to those in $\widetilde{I}\left(-s, w_{2 r, r}\left(\pi_{\psi}(\widetilde{\tau}) \otimes \widetilde{\tau}\right)\right)$. Hence the integral in (4.25) (or the integral in Proposition 4.3) equals

$$
\begin{aligned}
\int_{M(k) U(\mathbb{A}) \backslash G(\mathbb{A})} \Phi^{c}(g, s, \phi) & \left.\int_{\operatorname{Mat}_{r-l, 2 r}(\mathbb{A})} \int_{\left[V_{r, l}\right]} \widetilde{\theta}_{\varphi_{2 r+l}, l} \ell\left(\bar{p}_{2}, \bar{z}\right) \ell\left(\bar{p}_{1}\right) \widetilde{g}\right) \\
& \times \widetilde{\mathcal{M}}_{1}(\widetilde{\Phi})\left(v v^{-}\left(p_{1}\right) w \widetilde{g}\right) \psi_{r-l}(v) d v d p_{1} d g .
\end{aligned}
$$

By the Iwasawa decomposition $G(\mathbb{A})=P(\mathbb{A}) \mathrm{K}\left(P=P_{2 r, l}\right)$, we have

$$
M(k) U(\mathbb{A}) \backslash G(\mathbb{A})=[M(k) \backslash M(\mathbb{A})] \mathrm{K} .
$$

Recall that $M=\mathrm{GL}_{2 r} \times \mathrm{Sp}_{2 l}$ and write

$$
m=m(a, b)=m(a) m(b) .
$$

If we write $g=u m \mathrm{k}$, we have, in (4.30),

$$
\Phi^{c}(g, s, \phi)=\Phi(g, s, \phi)\left(1-\tau_{c}(H(g))\right)=\Phi(m \mathrm{k}, s, \phi)\left(1-\tau_{c}(H(m))\right)
$$

and

$$
\widetilde{\theta}_{\varphi_{2 r+l}, l}\left(\ell\left(\bar{p}_{2}, \bar{z}\right) \ell\left(\bar{p}_{1}\right) \widetilde{m}(a, b) \mathrm{k}\right)=|\operatorname{det} a|^{\frac{1}{2}} \gamma_{\psi}(\operatorname{det} a) \widetilde{\theta}_{\varphi_{2 r+l}, l}\left(\ell\left(\bar{p}_{2}, \bar{z}\right) \ell\left(\overline{p_{1} \cdot a}\right) \widetilde{m}(b) \mathrm{k}\right),
$$

where $\gamma_{\psi}$ is the Weil factor. Thus the inner integrations over $\operatorname{Mat}_{r-l, 2 r}(\mathbb{A})$ and $\left[V_{r, l}\right]$ of integral (4.30)

$$
\iint \widetilde{\theta}_{\varphi_{2 r+l}, l}\left(\ell\left(\bar{p}_{2}, \bar{z}\right) \ell\left(\bar{p}_{1}\right) \widetilde{m}(a, b) \mathrm{k}\right) \widetilde{\mathcal{M}}_{1}(\widetilde{\Phi})\left(v v^{-}\left(p_{1}\right) w \widetilde{m}(a, b) \mathrm{k}\right) \psi_{r-l}(v) d v d p_{1}
$$


equals

$$
\begin{gathered}
|\operatorname{det} a|^{l-r+\frac{1}{2}} \gamma_{\psi}(\operatorname{det} a) \int_{\operatorname{Mat}_{r-l, 2 r}(\mathbb{A})} \int_{\left[V_{r, l]}\right]} \widetilde{\theta}_{\varphi_{2 r+l}, l}\left(\ell\left(\bar{p}_{2}, \bar{z}\right) \ell\left(\bar{p}_{1}\right) \widetilde{m}(b) \mathrm{k}\right) \\
\times \widetilde{\mathcal{M}}_{1}(\widetilde{\Phi})\left(\widetilde{m}(a) v v^{-}\left(p_{1}\right) w \widetilde{m}(b) \mathrm{k}\right) \psi_{r-l}(v) d v d p_{1},
\end{gathered}
$$

where $\widetilde{m}(a)=\widetilde{m}\left(a, I_{2 r}\right), \widetilde{m}(b)=\widetilde{m}\left(I_{2 r}, b\right) \in \mathrm{GL}_{2 r}(\mathbb{A}) \times{\widetilde{\mathrm{Sp}_{2 r}}}_{(\mathbb{A})}$.

From the definition of $\Phi\left(h, s, \phi_{\pi_{\psi}(\widetilde{\tau}) \otimes \sigma}\right)$ and $\widetilde{\Phi}\left(\widetilde{g}, s, \widetilde{\phi}_{\pi_{\psi}(\widetilde{\tau}) \otimes \widetilde{\tau}}\right)$ as given in $\S 3$, we have

$$
\begin{aligned}
\Phi\left(m(a, b) \mathrm{k}, s, \phi_{\pi_{\psi}(\widetilde{\tau}) \otimes \sigma}\right) & =|\operatorname{det} a|^{s+r+l+\frac{1}{2}} \cdot \phi_{\pi_{\psi}(\widetilde{\tau}) \otimes \sigma}(m(a, b) \mathrm{k}) \\
\widetilde{\Phi}\left(\widetilde{m}(a, b) \widetilde{\mathrm{k}}, s, \widetilde{\phi}_{\pi_{\psi}(\widetilde{\tau}) \otimes \widetilde{\tau}}\right) & =|\operatorname{det} a|^{s+2 r+\frac{1}{2}} \cdot \gamma_{\psi}^{-1}(\operatorname{det} a) \cdot \widetilde{\phi}_{\pi_{\psi}(\widetilde{\tau}) \otimes \widetilde{\tau}}(\widetilde{m}(a, b) \widetilde{\mathrm{k}}) .
\end{aligned}
$$

Hence, taking into account the Jacobian which results from the Iwasawa decomposition, the integral in (4.30) can be expressed as

$$
\begin{array}{ll}
\int_{\mathrm{K}} & \int_{\operatorname{Mat}_{r-l, 2 r}(\mathbb{A})} \int_{\left[\mathrm{GL}_{2 r}\right]}|\operatorname{det} a|^{s-\frac{1}{2}}\left(1-\tau_{c}(H(a))\right) \\
(32) & \times \int_{\left[\mathrm{Sp}_{2 l}\right]} \phi_{\pi_{\psi}(\widetilde{\tau}) \otimes \sigma}(m(a, b) \mathrm{k}) \mathcal{F}^{\psi}\left(\widetilde{\phi}_{\pi_{\psi}(\widetilde{\tau}) \otimes \widetilde{\tau}}\right)\left(m(a, b) v^{-}\left(p_{1}\right) w \mathrm{k}\right) d b d a d p_{1} d \mathrm{k}
\end{array}
$$

where the function $\mathcal{F}^{\psi}\left(\widetilde{\phi}_{\pi_{\psi}(\widetilde{\tau}) \otimes \widetilde{\tau}}\right)\left(m(a, b) v^{-}\left(p_{1}\right) w \mathrm{k}\right)$ is given by

$$
\int_{\left[V_{r, l}\right]} \tilde{\theta}_{\varphi_{2 r+l}, l}\left(\ell\left(\bar{p}_{2}, \bar{z}\right) \ell\left(\bar{p}_{1}\right) \widetilde{m}(b) \mathrm{k}\right) \widetilde{\phi}\left(v \widetilde{m}(a, b) v^{-}\left(p_{1}\right) w \mathrm{k}\right) \psi_{r-l}(v) d v
$$

for $v=v\left(n, p_{2}, z\right)$ and $\widetilde{\phi}=\widetilde{\phi}_{\pi_{\psi}(\widetilde{\tau}) \otimes \tilde{\tau}}$. We consider the Langlands decomposition for $\mathrm{GL}_{2 r}(\mathbb{A})$

$$
\mathrm{GL}_{2 r}(\mathbb{A})=\mathrm{GL}_{2 r}(\mathbb{A})^{1} \cdot A^{+},
$$

and we set $\mathrm{PM}_{2 r, l}=\left(Z_{\mathrm{GL}_{2 r}}(\mathbb{A}) \mathrm{GL}_{2 r}(k) \backslash \mathrm{GL}_{2 r}(\mathbb{A})\right) \times\left(\mathrm{Sp}_{2 l}(k) \backslash \mathrm{Sp}_{2 l}(\mathbb{A})\right)$. Let $d$ be the number of the real archimedean places of the number field $k$. Then integral (4.32) equals

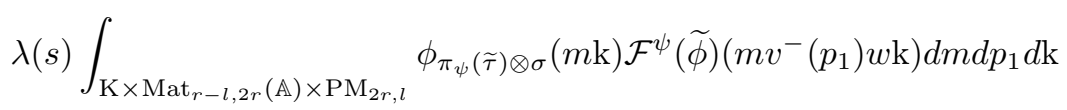

where the function $\lambda(s)$ is defined as

$$
\lambda(s):=\operatorname{vol}\left(\mathbb{A}^{1} / k^{\times}\right) \int_{\mathbb{R}^{+}}|t|^{2 r d\left(s-\frac{1}{2}\right)}\left(1-\tau_{c}(t)\right) d t^{\times} .
$$

It is easy to see that

$$
\int_{\mathbb{R}^{+}}|t|^{2 r d\left(s-\frac{1}{2}\right)}\left(1-\tau_{c}(t)\right) d t^{\times}=\frac{c^{2 r d\left(s-\frac{1}{2}\right)}}{2 r d\left(s-\frac{1}{2}\right)}
$$

which has a simple pole at $s=\frac{1}{2}$. In (4.34) we are left with

$$
\int_{\mathrm{K} \times \mathrm{Mat}_{r-l, 2 r}(\mathbb{A}) \times \mathrm{PM}_{2 r, l}} \phi_{\pi_{\psi}(\widetilde{\tau}) \otimes \sigma}(m \mathrm{k}) \mathcal{F}^{\psi}(\widetilde{\phi})\left(m v^{-}\left(p_{1}\right) w \mathrm{k}\right) d m d p_{1} d \mathrm{k}
$$

which is holomorphic in $s$. Hence we obtain the main identity. 
Theorem 4.4. When $s_{0} \neq \frac{1}{2}$, the period

$$
\mathcal{P}_{3 r, r-l}\left(E_{s_{0}}\left(\cdot, \phi_{\pi_{\psi}(\widetilde{\tau}) \otimes \sigma}\right), \widetilde{E}_{1}\left(\cdot, \widetilde{\phi}_{\pi_{\psi}(\widetilde{\tau}) \otimes \widetilde{\tau}}\right), \varphi_{2 r+l}\right)
$$

is identically zero. When $s_{0}=\frac{1}{2}$, the period

$$
\mathcal{P}_{3 r, r-l}\left(E_{\frac{1}{2}}\left(\cdot, \phi_{\pi_{\psi}(\widetilde{\tau}) \otimes \sigma}\right), \widetilde{E}_{1}\left(\cdot, \widetilde{\phi}_{\pi_{\psi}(\widetilde{\tau}) \otimes \widetilde{\tau}}\right), \varphi_{2 r+l}\right)
$$

is equal to

$$
\mathfrak{c} \times \int_{\mathrm{K} \times \mathrm{Mat}_{r-l, 2 r}(\mathbb{A}) \times \mathrm{PM}_{2 r, l}} \phi_{\pi_{\psi}(\widetilde{\tau}) \otimes \sigma}(m \mathrm{k}) \mathcal{F}^{\psi}\left(\widetilde{\phi}_{\pi_{\psi}(\widetilde{\tau}) \otimes \tilde{\tau}}\right)\left(m v^{-}\left(p_{1}\right) w \mathrm{k}\right) d m d p_{1} d \mathrm{k}
$$

where $\mathrm{PM}_{2 r, l}=\left(Z_{\mathrm{GL}_{2 r}}(\mathbb{A}) \mathrm{GL}_{2 r}(k) \backslash \mathrm{GL}_{2 r}(\mathbb{A})\right) \times\left(\mathrm{Sp}_{2 l}(k) \backslash \mathrm{Sp}_{2 l}(\mathbb{A})\right)$ and the constant $\mathfrak{c}$ is equal to the residue at $s=\frac{1}{2}$ of $\lambda(s)$, which is $\frac{\operatorname{vol}\left(\mathbb{A}^{1} / k^{\times}\right)}{2 r d}$.

Remark 4.5. From formulas (4.34) and (4.35), the period

$$
\mathcal{P}_{3 r, r-l}\left(\mathcal{E}_{1}, \widetilde{E}_{1}\left(\cdot, \widetilde{\phi}_{\pi_{\psi}(\widetilde{\tau}) \otimes \tilde{\tau}}\right), \varphi_{2 r+l}\right)
$$

has meromorphic continuation to the whole complex plane.

This completes, for the period $\mathcal{P}_{3 r, r-l}\left(\mathcal{E}_{1}, \widetilde{E}_{1}\left(\cdot, \widetilde{\phi}_{\pi_{\psi}(\widetilde{\tau}) \otimes \tilde{\tau}}\right), \varphi_{2 r+l}\right)$, the proof of Proposition 4.1. The proof for the period $\mathcal{P}_{3 r, r-l}\left(\mathcal{E}_{2}, \widetilde{E}_{1}\left(\cdot, \widetilde{\phi}_{\pi_{\psi}(\widetilde{\tau}) \otimes \tau}\right), \varphi_{2 r+l}\right)$ follows from the same argument (as in GJR01]). Hence the proof for Proposition 4.1 is now completed.

Remark 4.6. There is an analog of Theorem 4.4 for the case of $l \geq r$. In this case one has that $4 r+2 l \geq 6 r$. One has to consider the Fourier-Jacobi coefficient of the residue $E_{\frac{1}{2}}\left(g, \phi_{\pi_{\psi}(\widetilde{\tau}) \otimes \sigma}\right)$ on $\operatorname{Sp}_{4 r+2 l}(\mathbb{A})$. Note that this Fourier-Jacobi coefficient is

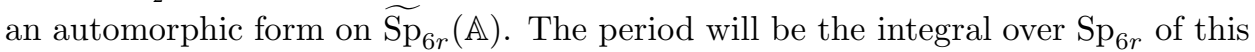
Fourier-Jacobi coefficient against the residue $\widetilde{E}_{1}\left(\cdot, \widetilde{\phi}_{\pi_{\psi}(\widetilde{\tau}) \otimes \widetilde{\tau}}\right)$ on $\widetilde{\mathrm{Sp}}_{6 r}(\mathbb{A})$. Again the product of two genuine automorphic forms is no longer genuine, so the integration can be taken over the linear group $\mathrm{Sp}_{6 r}$. In order to avoid extra notation, we omit the details here for $l \geq r$.

\section{A sufficient CONDition for $L\left(\frac{1}{2}, \pi_{1} \times \pi_{2}\right) \neq 0$}

In this section we prove Theorem 5.1, which gives a sufficient condition for $L\left(\frac{1}{2}, \pi_{1} \times \pi_{2}\right)$ to be nonzero.

Let $\pi_{1}$ be an irreducible unitary cuspidal automorphic representation of $\mathrm{GL}_{2 l+1}(\mathbb{A})$ such that the symmetric square $L$-function $L\left(s, \pi_{1}, \mathrm{Sym}^{2}\right)$ has a pole at $s=1$, and let $\sigma$ be an irreducible unitary generic cuspidal automorphic representation of $\operatorname{Sp}_{2 l}(\mathbb{A})$ which lifts functorially to $\pi_{1}$. Let $\pi_{2}$ be an irreducible unitary cuspidal automorphic representation of $\mathrm{GL}_{2 r}(\mathbb{A})$ with the properties that the exterior square $L$-function $L\left(s, \pi_{2}, \Lambda^{2}\right)$ has a pole at $s=1$ and the standard $L$-function $L\left(s, \pi_{2}\right)$ does not vanish at $s=\frac{1}{2}$. Take $\widetilde{\tau}$ to be an irreducible unitary generic cuspidal automorphic representation of $\widetilde{\mathrm{Sp}}_{2 r}(\mathbb{A})$ which has the $\psi$-transfer $\pi_{2}$. Note that the existence of $\sigma$ and $\widetilde{\tau}$ is established by the automorphic descent method (GRS99c], GRS01, and [S02]).

Theorem 5.1. Let $\phi_{\sigma} \in V_{\sigma}$ and $\widetilde{\phi}_{\tilde{\tau}} \in V_{\widetilde{\tau}}$. If the period integral

$$
\mathcal{P}_{r, r-l}\left(\phi_{\sigma}, \widetilde{\phi}_{\tilde{\tau}}, \varphi_{l}\right)(r \geq l) \quad \text { or } \quad \widetilde{\mathcal{P}}_{l, l-r}\left(\widetilde{\phi}_{\tilde{\tau}}, \phi_{\sigma}, \varphi_{r}\right)(r \leq l)
$$


attached to $(\sigma, \widetilde{\tau}, \psi)$, as defined in (2.12) and (2.13), is nonzero, then the central value of the Rankin-Selberg product $L$-function $L\left(\frac{1}{2}, \pi_{1} \times \pi_{2}\right)$ is nonzero.

Remark 5.2. If the irreducible unitary cuspidal automorphic representation $\pi_{2}$ of $\mathrm{GL}_{2 r}(\mathbb{A})$ has the properties that the exterior square $L$-function $L\left(s, \pi_{2}, \Lambda^{2}\right)$ has a pole at $s=1$ and the standard $L$-function $L\left(s, \pi_{2}\right)$ vanishes at $s=\frac{1}{2}$, the lifting theory is more complicated and we hope to deal with this case in a future publication.

By (2.12) and (2.13) the periods are defined as follows. If $l \geq r$, we have

$$
\widetilde{\mathcal{P}}_{l, l-r}\left(\widetilde{\phi}_{\widetilde{\tau}}, \phi_{\sigma}, \varphi_{r}\right)=\int_{\operatorname{Sp}_{2 r}(k) \backslash \operatorname{Sp}_{2 r}(\mathbb{A})} \widetilde{\phi}_{\widetilde{\tau}}(\widetilde{g}) \widetilde{\mathcal{F}}_{\varphi_{r}}^{\psi}\left(\phi_{\sigma}\right)(\widetilde{g}) d g,
$$

where $\phi_{\sigma} \in V_{\sigma}, \widetilde{\phi}_{\widetilde{\tau}} \in V_{\widetilde{\tau}}$, and $\varphi_{r}$ is a Bruhat-Schwartz function on $\mathbb{A}^{r}$ (i.e. $\varphi_{r} \in$ $\left.\mathcal{S}\left(\mathbb{A}^{r}\right)\right)$. Note that the product of two genuine functions is no longer genuine, and hence the integration is taken over $\operatorname{Sp}_{2 r}(k) \backslash \operatorname{Sp}_{2 r}(\mathbb{A})$. If $l \leq r$, we have

$$
\mathcal{P}_{r, r-l}\left(\phi_{\sigma}, \widetilde{\phi}_{\tilde{\tau}}, \varphi_{l}\right)=\int_{\operatorname{Sp}_{2 l}(k) \backslash \operatorname{Sp}_{2 l}(\mathbb{A})} \phi_{\sigma}(g) \mathcal{F}_{\varphi_{l}}^{\psi}\left(\widetilde{\phi}_{\tilde{\tau}}\right)(g) d g
$$

where $\phi_{\sigma} \in V_{\sigma}, \widetilde{\phi}_{\widetilde{\tau}} \in V_{\widetilde{\tau}}$, and $\varphi_{l} \in \mathcal{S}\left(\mathbb{A}^{l}\right)$.

We prove this theorem in detail for the case where $l \leq r$ using Theorem 4.4. The other case where $l \geq r$ can be proved by the same idea and the same argument. We omit the details here.

When $l \leq r$, the period is $\mathcal{P}_{r, r-l}\left(\phi_{\sigma}, \widetilde{\phi}_{\widetilde{\tau}}, \varphi_{l}\right)$ as in (5.2). Denote by $\pi_{\psi}(\widetilde{\tau})$ the image of the $\psi$-transfer of $\widetilde{\tau}$ from $\widetilde{\mathrm{Sp}}_{2 r}(\mathbb{A})$ to $\mathrm{GL}_{2 r}(\mathbb{A})$. By our assumption at the beginning of this section, we have $\pi_{2}=\pi_{\psi}(\widetilde{\tau})$. To use Theorem 4.4, we consider the generic cuspidal data $\left(P_{2 r, l}, \pi_{\psi}(\widetilde{\tau}) \otimes \sigma\right)$ of $\operatorname{Sp}_{4 r+2 l}(\mathbb{A})$ and $\left(\widetilde{P}_{2 r, r}, \pi_{\psi}(\widetilde{\tau}) \otimes \widetilde{\tau}\right)$ of $\widetilde{\mathrm{Sp}}_{6 r}(\mathbb{A})$, respectively. We may assume that

$$
\begin{aligned}
\phi_{\pi_{\psi}(\widetilde{\tau}) \otimes \sigma} & =\phi_{\pi_{\psi}(\widetilde{\tau})} \otimes \phi_{\sigma}, \\
\widetilde{\phi}_{\pi_{\psi}(\widetilde{\tau}) \otimes \widetilde{\tau}} & =\bar{\phi}_{\pi_{\psi}(\widetilde{\tau})} \otimes \widetilde{\phi}_{\widetilde{\tau}},
\end{aligned}
$$

where $\bar{\phi}_{\pi_{\psi}(\widetilde{\tau})}$ is the complex conjugate of $\phi_{\pi_{\psi}(\widetilde{\tau})}$. Recall from (3.2), (3.3) that the function $\Phi\left(g, s, \phi_{\pi_{\psi}(\widetilde{\tau}) \otimes \sigma}\right)$ is given by

$$
\Phi\left(g, s, \phi_{\pi_{\psi}(\widetilde{\tau}) \otimes \sigma}\right)=\phi_{\pi_{\psi}(\widetilde{\tau}) \otimes \sigma}(g) \exp \left\langle s+\rho_{P_{2 r, l}}, H_{P_{2 r, l}}(g)\right\rangle
$$

where the parameter $s$ is normalized as in (3.3) (i.e., $s$ is identified with $s \tilde{\alpha}_{2 r}$ ), and the function $\widetilde{\Phi}\left(\widetilde{g}, s, \widetilde{\phi}_{\pi_{\psi}(\widetilde{\tau}) \otimes \widetilde{\tau}}\right)$ is given by

$$
\widetilde{\Phi}\left(\widetilde{g}, s, \widetilde{\phi}_{\pi_{\psi}(\widetilde{\tau}) \otimes \widetilde{\tau}}\right)=\widetilde{\phi}_{\pi_{\psi}(\widetilde{\tau}) \otimes \tilde{\tau}}(\widetilde{g}) \exp \left\langle s+\rho_{\widetilde{P}_{2 r, r}}, H_{\widetilde{P}_{2 r, r}}(\widetilde{g})\right\rangle
$$

following (3.13) and (3.14).

We need the following proposition to finish the proof of Theorem 5.1.

Proposition 5.3. If the period

$$
\mathcal{P}_{r, r-l}\left(\phi_{\sigma}, \widetilde{\phi}_{\tilde{\tau}}, \varphi_{l}\right)
$$

does not vanish for some given $\phi_{\sigma} \in V_{\sigma}, \widetilde{\phi}_{\widetilde{\tau}} \in V_{\widetilde{\tau}}$, and $\phi_{\pi_{\psi}(\widetilde{\tau})} \in V_{\pi_{\psi}(\widetilde{\tau})}$, then integral (4.35), which equals

$$
\int_{\mathrm{K} \times \operatorname{Mat}_{r-l, 2 r}(\mathbb{A}) \times \mathrm{PM}_{2 r, l}} \phi_{\pi_{\psi}(\widetilde{\tau}) \otimes \sigma}(m \mathrm{k}) \mathcal{F}^{\psi}\left(\widetilde{\phi}_{\pi_{\psi}(\widetilde{\tau}) \otimes \widetilde{\tau}}\left(m v^{-}\left(p_{1}\right) w \mathrm{k}\right) d m d p_{1} d \mathrm{k}\right.
$$


does not vanish for the corresponding data defined in (5.5) and (5.6), where the function $\mathcal{F}^{\psi}\left(\widetilde{\phi}_{\pi_{\psi}(\widetilde{\tau}) \otimes \tilde{\tau}}\right)$ is defined as in (4.33).

Proof. Recall from (5.2), (2.11), and (2.13) that the period $\mathcal{P}_{r, r-l}\left(\phi_{\sigma}, \widetilde{\phi}_{\tilde{\tau}}, \varphi_{l}\right)$ equals

$$
\int_{\left[\mathrm{Sp}_{2 l}\right]} \phi_{\sigma}(g) \int_{\left[V_{r, l}\right]} \widetilde{\theta}_{\varphi_{l}}^{\psi}\left(\ell_{l}(v) \widetilde{g}\right) \widetilde{\phi}_{\tilde{\tau}}(v \widetilde{g}) \psi_{r-l}(v) d v d g .
$$

It defines a continuous functional on the space of

$$
V_{\sigma} \otimes\left(\Theta_{l}^{\psi} \otimes V_{\widetilde{\tau}}\right)^{V_{l}, \psi_{r-l},}
$$

where $\Theta_{l}^{\psi}$ is the space generated by the theta functions $\theta_{\varphi_{l}}^{\psi}$ with $\varphi_{l} \in \mathcal{S}\left(\mathbb{A}^{l}\right)$ and $\left(\Theta_{l}^{\psi} \otimes V_{\widetilde{\tau}}\right)^{V_{l}, \psi_{r-l}}$ is the space generated by the Fourier-Jacobi coefficients of automorphic forms in $\widetilde{\tau}$.

Recall from (4.33) that the function $\mathcal{F}^{\psi}\left(\widetilde{\phi}_{\pi_{\psi}(\widetilde{\tau}) \otimes \tilde{\tau}}\right)\left(m(a, b) v^{-}\left(p_{1}\right) w \mathrm{k}\right)$ equals

$$
\int_{\left[V_{r, l}\right]} \widetilde{\theta}_{\varphi_{2 r+l}, l}\left(\ell\left(\bar{p}_{2}, \bar{z}\right) \ell\left(\bar{p}_{1}\right) \widetilde{m}(b) \mathrm{k}\right) \widetilde{\phi}_{\pi_{\psi}(\widetilde{\tau}) \otimes \widetilde{\tau}}\left(v \widetilde{m}(a, b) v^{-}\left(p_{1}\right) w \mathrm{k}\right) \psi_{r-l}(v) d v .
$$

It is clear that $\mathcal{S}\left(\mathbb{A}^{2 r+l}\right)=\mathcal{S}\left(\mathbb{A}^{2 r}\right) \hat{\otimes} \mathcal{S}\left(\mathbb{A}^{l}\right)$. If we take $\varphi_{2 r+l}=\varphi_{2 r} \otimes \varphi_{l}$ (separation of variables), then we have

$$
\widetilde{\theta}_{\varphi_{2 r+l}, l}\left(\ell\left(\bar{p}_{2}, \bar{z}\right) \ell\left(\bar{p}_{1}\right) \widetilde{g}\right)=\varphi_{2 r}\left(\ell\left(\bar{p}_{1}\right)\right) \cdot \widetilde{\theta}_{\varphi_{l}}^{\psi}\left(\ell\left(\bar{p}_{2}, \bar{z}\right) \widetilde{g}\right)
$$

for $\widetilde{g} \in \widetilde{\mathrm{Sp}}_{2 l}(\mathbb{A})$. For any fixed $\varphi_{2 r} \in \mathcal{S}\left(\mathbb{A}^{2 r}\right)$, we consider all Bruhat-Schwartz functions

$$
\varphi_{2 r+l}=\varphi_{2 r} \otimes \varphi_{l} \in \mathcal{S}\left(\mathbb{A}^{2 r+l}\right),
$$

with $\varphi_{l} \in \mathcal{S}\left(\mathbb{A}^{l}\right)$. It follows that the space generated by $\widetilde{\theta}_{\varphi_{2 r+l}, l}\left(\ell\left(\bar{p}_{2}, \bar{z}\right) \widetilde{g}\right.$ ) (with a fixed $\varphi_{2 r} \in \mathcal{S}\left(\mathbb{A}^{2 r}\right)$ and all $\left.\varphi_{l} \in \mathcal{S}\left(\mathbb{A}^{l}\right)\right)$ is the same as the space $\Theta_{l}^{\psi}$ (generated by all $\left.\widetilde{\theta}_{\varphi_{l}}^{\psi}\left(\ell\left(\bar{p}_{2}, \bar{z}\right) \widetilde{g}\right)\right)$ as automorphic representations of the Jacobi group $\widetilde{\operatorname{Sp}}_{2 l}(\mathbb{A}) \ltimes H_{l}(\mathbb{A})$, where $H_{l}$ is the Heisenberg group generated by all $\ell\left(\bar{p}_{2}, \bar{z}\right)$. In the following we may assume that $\varphi_{2 r}$ is supported in a small neighborhood of zero. This is needed for the nonvanishing of $\mathcal{I}(\Psi)$ in (5.10) below, in particular for the integration in variable $p_{1}$. It follows that the nonvanishing of the period $\mathcal{P}_{r, r-l}\left(\phi_{\sigma}, \widetilde{\phi}_{\widetilde{\tau}}, \varphi_{l}\right)$ is equivalent to the nonvanishing of the integral

$$
\int_{\left[\mathrm{Sp}_{2 l}\right]} \phi_{\sigma}(b) \int_{\left[V_{r, l}\right]} \widetilde{\theta}_{\varphi_{2 r+l}, l}\left(\ell\left(\bar{p}_{2}, \bar{z}\right) \widetilde{b}\right) \widetilde{\phi}_{\widetilde{\tau}}(v \widetilde{b}) \psi_{r-l}(v) d v d b .
$$

It is clear that the integral

$$
\int_{Z_{\mathrm{GL}_{2 r}(\mathbb{A}) \mathrm{GL}_{2 r}(k) \backslash \mathrm{GL}_{2 r}(\mathbb{A})}} \phi_{\pi_{\psi}(\widetilde{\tau})}(a) \overline{\phi_{\pi_{\psi}(\widetilde{\tau})}}(a) d a
$$

is not zero for some choice of $\phi_{\pi_{\psi}(\widetilde{\tau})}$ and $\overline{\phi_{\pi_{\psi}(\widetilde{\tau})}}$. Using (5.3) and (5.4), the nonvanishing of the combination of the above two integrals is equivalent to the nonvanishing of the integral

$$
\int_{\mathrm{PM}_{2 r, l}} \phi_{\pi_{\psi}(\widetilde{\tau}) \otimes \sigma}(m) \mathcal{F}^{\psi}\left(\widetilde{\phi}_{\pi_{\psi}(\widetilde{\tau}) \otimes \widetilde{\tau}}\right)(m) d m
$$


where $\mathrm{PM}_{2 r, l}=\left(Z_{\mathrm{GL}_{2 r}}(\mathbb{A}) \mathrm{GL}_{2 r}(k) \backslash \mathrm{GL}_{2 r}(\mathbb{A})\right) \times\left(\operatorname{Sp}_{2 l}(k) \backslash \mathrm{Sp}_{2 l}(\mathbb{A})\right)$ as in (4.34), and for $m=m(a, b)$,

$$
\mathcal{F}^{\psi}\left(\widetilde{\phi}_{\pi_{\psi}(\widetilde{\tau}) \otimes \widetilde{\tau}}\right)(m)=\int_{\left[V_{r, l}\right]} \widetilde{\theta}_{\varphi_{2 r+l}, l}\left(\ell\left(\bar{p}_{2}, \bar{z}\right) \widetilde{m}(b)\right) \widetilde{\phi}_{\pi_{\psi}(\tilde{\tau}) \otimes \widetilde{\tau}}(m(a) v \widetilde{m}(b)) \psi_{r-l}(v) d v .
$$

Hence (5.8) gives rise to a nonzero continuous linear functional $\mathcal{J}$ on the space

$$
V_{\pi_{\psi}(\widetilde{\tau}) \otimes \sigma} \otimes\left[V_{\pi_{\psi}(\widetilde{\tau})} \otimes\left(\Theta_{l}^{\psi} \otimes \widetilde{\tau}\right)^{V_{r, l}, \psi_{r-l}}\right] .
$$

It remains to show that there are some choices of data, so that the integration over the maximal compact subgroup $\mathrm{K}$ of $\mathrm{Sp}_{4 r+2 l}(\mathbb{A})$ and over the variable $p_{1}$ of the functional in (5.8) will be nonzero. To do so, we have to realize the relevant representations in different models of functions. For example, for the integration over $\mathrm{K}$, one has to realize the induced representation in the compact model, while for the integration over variable $p_{1}$, one has to realize the parabolic induced representation from $P=M N$ in the model of functions over the opposite unipotent radical $N^{-}$. The argument has been used in [JR92], JJng98a, [GJR01, GJR03. and GJR. We sketch the main ideas following [GJR01.

As in the proof of Theorem 3.2 in GJR01, we consider the function

$$
\Psi: \mathrm{K} \times \operatorname{Mat}_{r-l, 2 r}(\mathbb{A}) \rightarrow V_{\pi_{\psi}(\widetilde{\tau}) \otimes \sigma} \otimes\left[V_{\pi_{\psi}(\widetilde{\tau})} \otimes\left(\Theta_{l}^{\psi} \otimes \widetilde{\tau}\right)^{V_{r, l}, \psi_{r-l}}\right]
$$

with left quasi-invariance

$$
\Psi(p \mathrm{k})=\rho(p) \Psi(\mathrm{k})
$$

for $p \in P_{2 r, l}(\mathbb{A}) \cap \mathrm{K}$. Here $\rho(p)$ denotes the representation in the space (5.9). Define

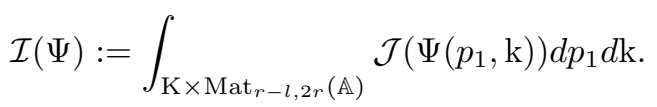

Then for a given $\phi \otimes \mathcal{F}^{\psi}(\widetilde{\phi})=\phi_{\pi_{\psi}(\widetilde{\tau}) \otimes \sigma} \otimes \mathcal{F}^{\psi}\left(\widetilde{\phi}_{\pi_{\psi}(\widetilde{\tau}) \otimes \tilde{\tau}}\right)$ in the space (5.9) of the representation $\rho$, there is a smooth function $\Psi$ such that

$$
\begin{aligned}
\mathcal{I}(\Psi) & =\int_{\mathrm{K} \times \mathrm{Mat}_{r-l, 2 r}(\mathbb{A})} \mathcal{J}\left(\Psi\left(p_{1}, \mathrm{k}\right)\right) d p_{1} d \mathrm{k} \\
& =\int_{\mathrm{K} \times \operatorname{Mat}_{r-l, 2 r}(\mathbb{A}) \times \mathrm{PM}_{2 r, l}} \phi(m \mathrm{k}) \mathcal{F}^{\psi}(\widetilde{\phi})\left(m v^{-}\left(p_{1}\right) w \mathrm{k}\right) d m d p_{1} d \mathrm{k} .
\end{aligned}
$$

To finish the proof of this proposition, it suffices to show that one can choose the function $\Psi$ with restricted support so that the integral $\mathcal{I}(\Psi)$ is nonzero.

Recall that $U_{2 r, r}$ is the unipotent radical of the maximal parabolic subgroup $P_{2 r, r}$ of $\mathrm{Sp}_{6 r}$. Since the integration in the variable $v^{-}\left(p_{1}\right)$ is in a subgroup of $U_{2 r, r}^{-}$ (the opposite of $U_{2 r, r}$ ), we can choose a section $\widetilde{\Phi}$ with the property that it is compactly supported modulo $P_{2 r, r}(\mathbb{A})$ as a function of $v^{-}\left(p_{1}\right)$ and compatible with the support of $\varphi_{2 r}$ as remarked before. Hence the integration in $p_{1}$ of (5.10) is nonzero. Since the variable $\mathrm{k}$ is independent of the variable $p_{1}$, the nonvanishing of the integration in variable $\mathrm{k}$ is proved the same way as in the proof of Theorem 3.2 in GJR01.

We now return to the proof of Theorem 5.1. By Proposition 5.3 and Theorem 4.4 , if the period $\mathcal{P}_{r, r-l}\left(\phi_{\sigma}, \widetilde{\phi}_{\tilde{\tau}}, \varphi_{l}\right)$ does not vanish for some given $\phi_{\sigma} \in V_{\sigma}, \widetilde{\phi}_{\tilde{\tau}} \in V_{\tilde{\tau}}$, and $\phi_{\pi_{\psi}(\widetilde{\tau})} \in V_{\pi_{\psi}(\widetilde{\tau})}$, then the period

$$
\mathcal{P}_{3 r, r-l}\left(E_{\frac{1}{2}}\left(\cdot, \phi_{\pi_{\psi}(\widetilde{\tau}) \otimes \sigma}\right), \widetilde{E}_{1}\left(\cdot, \widetilde{\phi}_{\pi_{\psi}(\widetilde{\tau}) \otimes \widetilde{\tau}}\right), \varphi_{2 r+l}\right)
$$


does not vanish for the corresponding data. In particular, this implies that the residue $E_{\frac{1}{2}}\left(g, \phi_{\pi_{\psi}(\widetilde{\tau}) \otimes \sigma}\right)$ does not vanish for the given data. Hence from Proposition 3.2 the product of $L$-functions

$$
L\left(s, \pi_{\psi}(\widetilde{\tau}) \times \sigma\right) L\left(2 s, \pi_{\psi}(\widetilde{\tau}), \Lambda^{2}\right)
$$

has a simple pole at $s=\frac{1}{2}$. Since the exterior square $L$-function

$$
L\left(s, \pi_{\psi}(\widetilde{\tau}), \Lambda^{2}\right)
$$

has a simple pole at $s=1$, the $L$-function $L\left(s, \pi_{\psi}(\widetilde{\tau}) \times \sigma\right)$ cannot vanish at $s=\frac{1}{2}$. Since $\pi_{2}=\pi_{\psi}(\widetilde{\tau})$ is the $\psi$-transfer of $\widetilde{\tau}$ from $\widetilde{\mathrm{Sp}}_{2 r}$ and $\pi_{1}$ is the image of the Langlands functorial lift of $\sigma$ from $\mathrm{Sp}_{2 l}$, we obtain that the tensor product $L$ function $L\left(s, \pi_{1} \times \pi_{2}\right)$ does not vanish at $s=\frac{1}{2}$. This completes the proof of Theorem 5.1.

\section{A necessary Condition for $L\left(\frac{1}{2}, \pi_{1} \times \pi_{2}\right) \neq 0$}

In this section we prove the second part of the Main Theorem. In this proof, we will make an assumption on the nonvanishing of certain Fourier coefficients of the residue $E_{\frac{1}{2}}\left(g, \phi_{\pi_{\psi}(\widetilde{\tau}) \otimes \sigma}\right)$. See Assumption (FC) below.

In order to state Assumption (FC), we recall briefly from GRS03 the notion of Fourier coefficients of automorphic forms associated to unipotent orbits. The $p$-adic version, which associates twisted Jacquet modules with unipotent orbits was well studied in [MW87]. Recall that the set $\mathcal{U}$ of all unipotent orbits in $\operatorname{Sp}_{2 n}(\mathbb{C})$ (or in any complex reductive group) is finite and partially ordered. The partial ordering in $\mathcal{U}$ is given by the 'included-in-closure' relation. Let $\mathcal{O}_{1}$ and $\mathcal{O}_{2}$ be two members in $\mathcal{U}$. One defines $\mathcal{O}_{1}<\mathcal{O}_{2}$ if $\mathcal{O}_{1}$ is included in the closure $\overline{\mathcal{O}_{2}}$ of $\mathcal{O}_{2}$. It is also known that unipotent orbits in $\mathrm{Sp}_{2 n}(\mathbb{C})$ are parameterized by partitions of symplectic type (see CM93 for example).

Let $\phi$ be an automorphic form on $\operatorname{Sp}_{2 n}(\mathbb{A})$. Following [GRS03, we let $\mathcal{O}(\phi)$ be the subset of $\mathcal{U}$ consisting of unipotent orbits with the property that $\mathcal{O} \in \mathcal{O}(\phi)$ if $\phi$ has a nonzero Fourier coefficient associated to $\mathcal{O}$ and for any unipotent orbit $\mathcal{O}^{\prime} \in \mathcal{U}$ bigger than $\mathcal{O}$, i.e. $\mathcal{O}^{\prime}>\mathcal{O}, \phi$ does not have any nonzero Fourier coefficient associated to $\mathcal{O}^{\prime}$. Let $\sigma$ be an irreducible automorphic representation of $\operatorname{Sp}_{2 n}(\mathbb{A})$ occurring in the discrete spectrum. We let $\mathcal{O}(\sigma)$ be $\mathcal{O}(\phi)$ for some nonzero $\phi \in \sigma$. Since $\sigma$ is irreducible, one can check easily that $\mathcal{O}(\phi)$ is independent of the choices of nonzero $\phi$ in $\sigma$.

Let $\sigma$ be an irreducible unitary generic cuspidal automorphic representation of $\mathrm{Sp}_{2 l}(\mathbb{A})$, and let $\widetilde{\tau}$ be an irreducible unitary generic cuspidal automorphic representation of $\widetilde{\operatorname{Sp}}_{2 r}(\mathbb{A})$. Assume that the image $\pi_{\psi}(\widetilde{\tau})$ of the $\psi$-transfer of $\widetilde{\tau}$ to $\mathrm{GL}_{2 r}(\mathbb{A})$ is cuspidal. We make the following assumption.

Assumption (FC). If the residue $E_{\frac{1}{2}}\left(g, \phi_{\pi_{\psi}(\tau) \otimes \sigma}\right)$ is nonzero, then it has a nonzero Fourier coefficient associated to the unipotent orbit $\mathcal{O}_{\left((2 r+2 l) 1^{2 r}\right)}$ parameterized by the symplectic partition $\left((2 r+2 l) 1^{2 r}\right)$.

Recall from (2.1) and (2.4) that the unipotent subgroup attached to the unipotent orbit $\mathcal{O}_{\left((2 r+2 l) 1^{2 r}\right)}$ of $\mathrm{Sp}_{4 r+2 l}$ can be expressed as (with $p$ replaced by $2 r+l$ 
and $i$ by $r+l$ )

$$
V_{\mathcal{O}_{((2 r+2 l) 12 r)}}=\left\{v=v(n, x, z)=\left(\begin{array}{ccc}
n & x & z \\
& I_{2 r} & x^{*} \\
& & n^{*}
\end{array}\right) \in \operatorname{Sp}_{4 r+2 l}\right\}
$$

where $n \in N_{r+l}$ and $x$ is an $(r+l) \times(2 r)$-matrix with zero bottom row. The character $\psi_{\mathcal{O}_{((2 r+2 l) 12 r)}^{a}}^{a}$ attached to $\mathcal{O}_{\left((2 r+2 l) 1^{2 r}\right)}$ is given (see (2.5)) by

$$
\psi_{\mathcal{O}_{((2 r+2 l) 12 r)}}^{a}(v)=\psi_{r+l}(n) \psi\left(a z_{r+l, 1}\right)=\psi\left(n_{1,2}+\cdots+n_{r+l-1, r+l}+a z_{r+l, 1}\right) .
$$

For an automorphic form $\phi$ of $\operatorname{Sp}_{4 r+2 l}(\mathbb{A})$, the $\psi_{\mathcal{O}_{((2 r+2 l) 12 r)}}^{a}$-Fourier coefficient of $\phi$ is defined by

$$
\int_{\left[V_{\mathcal{O}}((2 r+2 l) 12 r)\right.} \phi(v g) \overline{\psi_{\mathcal{O}_{((2 r+2 l) 12 r)}^{a}}^{a}(v)} d v .
$$

We remark that the $k$-rational orbits of $\mathcal{O}_{\left((2 r+2 l) 1^{2 r}\right)}$ are parameterized by the square classes of $a$ in $k^{\times}$. Assumption (FC) requires that the $\psi_{\mathcal{O}_{((2 r+2 l) 12 r)}}^{a}$-Fourier coefficient of the residue $E_{\frac{1}{2}}\left(g, \phi_{\pi_{\psi}(\widetilde{\tau}) \otimes \sigma}\right)$ is nonzero for at least one square class of $a$. In fact, we make a stronger conjecture.

Conjecture 6.1. If the residue $E_{\frac{1}{2}}\left(g, \phi_{\pi_{\psi}(\widetilde{\tau}) \otimes \sigma}\right)$ is nonzero, then it has the property that

$$
\mathcal{O}\left(E_{\frac{1}{2}}\left(\cdot, \phi_{\pi_{\psi}(\widetilde{\tau}) \otimes \sigma}\right)\right)=\mathcal{O}_{((2 r+2 l)(2 r))} .
$$

It is easy to check that Conjecture 6.1 implies Assumption (FC). In fact, first a $\psi_{\left.\mathcal{O}_{((2 r+2 l) 12 r}\right)}^{a}$-Fourier coefficient of the residue $E_{\frac{1}{2}}\left(g, \phi_{\pi_{\psi}(\widetilde{\tau}) \otimes \sigma}\right)$ is an automorphic form on $\widetilde{\mathrm{Sp}}_{2 r}(\mathbb{A})$, and a $\psi_{\mathcal{O}_{((2 r+2 l)(2 r))}^{a}}^{a}$-Fourier coefficient of the residue $E_{\frac{1}{2}}\left(g, \phi_{\pi_{\psi}(\widetilde{\tau}) \otimes \sigma}\right)$ produces a Whittaker-Fourier coefficient (i.e., associated to the regular unipotent orbit) of the $\psi_{\left.\mathcal{O}_{((2 r+2 l) 12 r}\right)}^{a}$-Fourier coefficient of the residue $E_{\frac{1}{2}}\left(g, \phi_{\pi_{\psi}(\widetilde{\tau}) \otimes \sigma}\right)$. Hence the nonvanishing of a $\psi_{\mathcal{O}_{((2 r+2 l)(2 r))}}^{a}$-Fourier coefficient of the residue $E_{\frac{1}{2}}\left(g, \phi_{\pi_{\psi}(\widetilde{\tau}) \otimes \sigma}\right)$ implies the nonvanishing of a $\psi_{\left.\mathcal{O}_{((2 r+2 l) 12 r}\right)}^{a}$-Fourier coefficient of $E_{\frac{1}{2}}\left(g, \phi_{\pi_{\psi}(\widetilde{\tau}) \otimes \sigma}\right)$.

By using the same argument as in Proposition 1 in [GRS], we can prove the following proposition, which supports the conjecture.

Proposition 6.2. Let $\mathcal{O}$ be a unipotent orbit of $\mathrm{Sp}_{4 r+2 l}$. If the unipotent orbit $\mathcal{O}$ is greater than or not related to the unipotent orbit $\mathcal{O}_{((2 r+2 l)(2 r))}$, then the residue $E_{\frac{1}{2}}\left(\cdot, \phi_{\pi_{\psi}(\widetilde{\tau}) \otimes \sigma}\right)$ has no nonzero Fourier coefficient associated to the unipotent orbit $\mathcal{O}$.

Proof. Let $\mathcal{O}=\mathcal{O}_{\left(n_{1} \ldots n_{q}\right)}$ be any unipotent orbit which is bigger than the orbit $\mathcal{O}_{((2 r+2 l)(2 r))}$ and such that the residue $E_{\frac{1}{2}}\left(g, \phi_{\pi_{\psi}(\widetilde{\tau}) \otimes \sigma}\right)$ has a nonzero Fourier coefficient associated to $\mathcal{O}$. Then $n_{1}>2 r+2 l$. Arguing as in Lemmas 2.4 and 2.6 in GRS03, we deduce that there is a number $p$ which is larger than $2 r+2 l$ such that the residue $E_{\frac{1}{2}}\left(g, \phi_{\pi_{\psi}(\widetilde{\tau}) \otimes \sigma}\right)$ has a nonzero Fourier coefficient associated to the unipotent orbit $\mathcal{O}_{\left((2 p) 1^{4 r+2 l-2 p}\right)}$. However, using Lemma 3 in [GRS] and Lemma 6.8 which we will prove in $\S 6.1$, it follows that $E_{\frac{1}{2}}\left(g, \phi_{\pi_{\psi}(\widetilde{\tau}) \otimes \sigma}\right)$ has no nonzero Fourier coefficients associated to the unipotent orbit $\mathcal{O}_{\left((2 p) 1^{4 r+2 l-2 p}\right)}$. Thus we derive a contradiction. 
The main result of this section is the theorem below. Let $\pi_{1}$ be an irreducible unitary cuspidal automorphic representation of $\mathrm{GL}_{2 l+1}(\mathbb{A})$ with the property that the symmetric square $L$-function $L\left(s, \pi_{1}, \mathrm{Sym}^{2}\right)$ has a pole at $s=1$, and let $\sigma$ be an irreducible unitary generic cuspidal automorphic representation of $\operatorname{Sp}_{2 l}(\mathbb{A})$ which lifts functorially to $\pi_{1}$. Let $\pi_{2}$ be an irreducible unitary cuspidal automorphic representation of $\mathrm{GL}_{2 r}(\mathbb{A})$ with the properties that the exterior square $L$-function $L\left(s, \pi_{2}, \Lambda^{2}\right)$ has a pole at $s=1$ and the standard $L$-function $L\left(s, \pi_{2}\right)$ does not vanish at $s=\frac{1}{2}$, and let $\widetilde{\tau}$ be an irreducible unitary genuine generic cuspidal automorphic representation of $\widetilde{\mathrm{Sp}}_{2 r}(\mathbb{A})$ which has the $\psi$-transfer to $\pi_{2}$.

Theorem 6.3. Assume that Assumption (FC) holds for the pair $(\widetilde{\tau}, \sigma)$. If the central value of the Rankin-Selberg product L-function $L\left(\frac{1}{2}, \pi_{1} \times \pi_{2}\right)$ is nonzero, then there exists an irreducible cuspidal automorphic representation $\sigma^{\prime}$ of $\operatorname{Sp}_{2 l}(\mathbb{A})$, which is nearly equivalent to $\sigma$, and there exists an irreducible cuspidal automorphic representation $\widetilde{\tau}^{\prime}$ of $\widetilde{\mathrm{Sp}}_{2 r}(\mathbb{A})$, which is nearly equivalent to $\widetilde{\tau}$, such that the period integral

$$
\mathcal{P}_{r, r-l}\left(\phi_{\sigma^{\prime}}, \widetilde{\phi}_{\widetilde{\tau}^{\prime}}, \varphi_{l}\right)(r \geq l) \quad \text { or } \quad \widetilde{\mathcal{P}}_{l, l-r}\left(\widetilde{\phi}_{\widetilde{\tau}^{\prime}}, \phi_{\sigma^{\prime}}, \varphi_{r}\right)(r \leq l)
$$

attached to $\left(\sigma^{\prime}, \widetilde{\tau}^{\prime}, \psi\right)$ is nonzero for some choices of the data.

We remark that we shall verify Assumption (FC) for the case $r \geq l=1$ in $\S 7$. Hence Theorem 6.3 holds unconditionally for the case $r \geq l=1$.

To prove Theorem 6.3 , we assume that $L\left(\frac{1}{2}, \pi_{1} \times \pi_{2}\right) \neq 0$. Since $\pi_{2}=\pi_{\psi}(\widetilde{\tau})$ and $\pi_{1}=\pi(\sigma)$, we have $L\left(\frac{1}{2}, \pi_{\psi}(\widetilde{\tau}) \times \sigma\right) \neq 0$ since $\pi_{1}=\pi(\sigma)$ is the image of the Langlands functorial lifting of $\sigma$. Hence the product of $L$-functions

$$
L\left(s, \pi_{\psi}(\widetilde{\tau}) \times \sigma\right) L\left(2 s, \pi_{\psi}(\widetilde{\tau}), \Lambda^{2}\right)
$$

has a simple pole at $s=\frac{1}{2}$. Proposition 3.2 implies that the residue

$$
E_{\frac{1}{2}}\left(g, \phi_{\pi_{\psi}(\widetilde{\tau}) \otimes \sigma}\right)
$$

does not vanish.

By Assumption (FC), we know that the residue $E_{\frac{1}{2}}\left(g, \phi_{\pi_{\psi}(\widetilde{\tau}) \otimes \sigma}\right)$ has a nonzero Fourier coefficient corresponding to the unipotent orbit $\mathcal{O}_{\left((2 r+2 l) 1^{2 r}\right)}$. That is, the integral

$$
\int_{\left[V_{\mathcal{O}_{((2 r+2 l) 12 r)}}\right]} E_{\frac{1}{2}}\left(v g, \phi_{\pi_{\psi}(\widetilde{\tau}) \otimes \sigma}\right) \overline{\psi_{\mathcal{O}_{((2 r+2 l) 12 r)}}^{a}(v)} d v
$$

is nonzero for some $a \in k^{\times}$. In order to match the square classes $a \in k^{\times}$associated to the Fourier coefficients which will be discussed below, we have to twist the residue $E_{\frac{1}{2}}\left(g, \phi_{\pi_{\psi}(\widetilde{\tau}) \otimes \sigma}\right)$ by a similitude element in $\mathrm{GSp}_{4 r+2 l}(k)$.

Let

$$
\mathrm{d}(a)=\left(\begin{array}{cc}
a I_{2 r+l} & 0 \\
0 & I_{2 r+l}
\end{array}\right)
$$

be a diagonal similitude element in $\operatorname{GSp}_{4 r+2 l}(k)$. Since the element d $(a)$ normalizes $\operatorname{Sp}_{4 r+2 l}(\mathbb{A})$, one may define the twist by $\mathrm{d}(a)$ of a representation $\Pi$ of $\operatorname{Sp}_{4 r+2 l}(\mathbb{A})$ by

$$
\Pi^{\mathrm{d}(a)}(g)=\Pi\left(\mathrm{d}(a) g \mathrm{~d}(a)^{-1}\right) .
$$

See Section II of Chapter 4 in [MVW87] for more discussions on twists of representations. Then one can check that the twist by $\mathrm{d}(a)$ of the residual representation generated by $E_{\frac{1}{2}}\left(g, \phi_{\pi_{\psi}(\widetilde{\tau}) \otimes \sigma}\right)$ is the residual representation generated by the 
residue $E_{\frac{1}{2}}\left(g, \phi_{\pi_{\psi}(\widetilde{\tau}) \otimes \sigma_{a}}\right)$. Here $\sigma_{a}$ is the twist of $\sigma$ by the similar element $\mathrm{d}(a)$ in $\mathrm{GSp}_{2 l}(k)$. It is easy to check that $\sigma_{a}$ is generic (with respect to the twisted generic character) and nearly equivalent to $\sigma$. This implies in particular that the residue $E_{\frac{1}{2}}\left(g, \phi_{\pi_{\psi}(\widetilde{\tau}) \otimes \sigma_{a}}\right)$ is nonzero and is nearly equivalent to $E_{\frac{1}{2}}\left(g, \phi_{\pi_{\psi}(\widetilde{\tau}) \otimes \sigma}\right)$. Now the residue $E_{\frac{1}{2}}\left(g, \phi_{\pi_{\psi}(\widetilde{\tau}) \otimes \sigma_{a}}\right)$ has the nonzero Fourier coefficient

$$
\int_{\left[V_{\mathcal{O}_{((2 r+2 l) 12 r)}}\right.} E_{\frac{1}{2}}\left(v g, \phi_{\pi_{\psi}(\widetilde{\tau}) \otimes \sigma_{a}} \overline{\psi_{\mathcal{O}_{((2 r+2 l) 12 r)}}(v)} d v\right.
$$

where $\psi_{\mathcal{O}_{((2 r+2 l) 12 r)}}=\psi_{\mathcal{O}_{((2 r+2 l) 12 r)}}^{1}$.

It follows from Lemma 1.1 in GRS03 that the nonvanishing of the Fourier coefficient in (6.2) is equivalent to the nonvanishing of the integral

$$
\widetilde{\mathcal{F}}_{\varphi_{2 r}}^{\psi_{r+l}}\left(E_{\frac{1}{2}}\right)(\widetilde{g})=\int_{\left[V_{2 r+l, r}\right]} \widetilde{\theta}_{\varphi_{2 r}}^{\psi}\left(\ell_{2 r}(v) \widetilde{g}\right) E_{\frac{1}{2}}\left(v \widetilde{g}, \phi_{\pi_{\psi}(\widetilde{\tau}) \otimes \sigma_{a}}\right) \overline{\psi_{r+l}(v)} d v .
$$

It is clear that $\widetilde{\mathcal{F}}_{\varphi_{2 r}}^{\psi_{r+l}}\left(E_{\frac{1}{2}}\right)(\widetilde{g})$ is an automorphic form on $\widetilde{\operatorname{Sp}}_{2 r}(\mathbb{A})$. Similar automorphic forms were studied in [GRS99c in the context of the automorphic descent map from GL to classical groups. However, the residue representation used in GRS99c was different.

Let $\widetilde{\tau}_{a}$ be the automorphic representation of $\widetilde{\operatorname{Sp}}_{2 r}(\mathbb{A})$ generated by all automorphic forms $\widetilde{\mathcal{F}}_{\varphi_{2 r}}^{\psi_{r+l}}\left(E_{\frac{1}{2}}\right)(\widetilde{g})$ defined in (6.3). We claim that

(1) the representation $\widetilde{\tau}_{a}$ is cuspidal;

(2) any irreducible summand of $\widetilde{\tau}_{a}$ is nearly equivalent to $\widetilde{\tau}$.

To prove the cuspidality of $\widetilde{\tau}_{a}$, it is enough to show that for any standard maximal parabolic subgroup $P_{j, r-j}=M_{j, r-j} U_{j, r-j}(1 \leq j \leq r)$, the constant terms of the automorphic forms $\widetilde{\mathcal{F}}_{\varphi_{2 r}}^{\psi_{r+l}}\left(E_{\frac{1}{2}}\right)(\widetilde{g})$ along the unipotent radical $U_{j, r-j}$ are all zero; i.e.,

$$
\int_{\left[U_{j, r-j}\right]} \widetilde{\mathcal{F}}_{\varphi_{2 r}}^{\psi_{r+l}}\left(E_{\frac{1}{2}}\right)(u \widetilde{g}) d u=0 .
$$

This is done as in [GRS99c, pages 844-847]. The idea is to take Fourier expansions of the constant term in (6.4) along some unipotent subgroups. In such Fourier expansions, there are two types of Fourier coefficients occurring in the summands. One type is the Fourier coefficient of the residue $E_{\frac{1}{2}}\left(g, \phi_{\pi_{\psi}(\widetilde{\tau}) \otimes \sigma_{a}}\right)$ associated to the unipotent orbit $\mathcal{O}_{\left((2 p) 1^{4 r+2 l-2 p}\right)}$ for $p>r+l$. It follows from Proposition 6.2 that the residue has no nonzero Fourier coefficients associated to such unipotent orbits. Another type is the Fourier coefficients which contain an integration of the constant terms of the residue $E_{\frac{1}{2}}\left(g, \phi_{\pi_{\psi}(\widetilde{\tau}) \otimes \sigma_{a}}\right)$ along the standard maximal parabolic subgroups other than $P_{2 r, l}$. These are zero due to the cuspidal support of the residue $E_{\frac{1}{2}}\left(g, \phi_{\pi_{\psi}(\widetilde{\tau}) \otimes \sigma_{a}}\right)$. This proves the cuspidality of $\widetilde{\tau}_{a}$.

Remark 6.4. The second assertion above is proved by studying the structure of the local unramified components of $\widetilde{\tau}_{a}$ and $\widetilde{\tau}$. This can be done in the same way as that of Proposition 5 in [GRS]. We will give some details at the end of $\S 6.1$, after certain preparation of local results.

By assertion (1) as proved above, the representation $\widetilde{\tau}_{a}$ is cuspidal. It follows that $\widetilde{\tau}_{a}$ can be written as a direct sum of irreducible cuspidal automorphic representations. Let $\widetilde{\tau}^{\prime}$ be one of the irreducible summands of $\widetilde{\tau}_{a}$. By assertion (2), $\widetilde{\tau}^{\prime}$ is 
nearly equivalent to $\widetilde{\tau}$. From (6.3), we have

$$
\int_{\left[\mathrm{Sp}_{2 r}\right]} \widetilde{\phi}_{\widetilde{\tau}^{\prime}}(\widetilde{g}) \int_{\left[V_{2 r+l, r}\right]} \widetilde{\theta}_{\varphi_{2 r}}^{\psi}\left(\ell_{2 r}(v) \widetilde{g}\right) E_{\frac{1}{2}}\left(v \widetilde{g}, \phi_{\pi_{\psi}(\widetilde{\tau}) \otimes \sigma_{a}}\right) \overline{\psi_{r+l}(v)} d v d \widetilde{g}
$$

does not vanish for some choices of data. We note here that the irreducible cuspidal automorphic representation $\widetilde{\tau}^{\prime}$ of $\widetilde{\mathrm{Sp}}_{2 r}(\mathbb{A})$ is nearly equivalent to $\widetilde{\tau}$, and the irreducible generic cuspidal automorphic representation $\sigma_{a}$ of $\mathrm{Sp}_{2 l}(\mathbb{A})$ is nearly equivalent to $\sigma$.

Remark 6.5. Let us mention that if Conjecture 6.1 holds, then $\widetilde{\tau}^{\prime}$ will be generic.

In integral (6.5), we replace the residue $E_{\frac{1}{2}}\left(\cdot, \phi_{\pi_{\psi}(\widetilde{\tau}) \otimes \sigma_{a}}\right)$ by the Eisenstein series $E\left(g, s, \phi_{\pi_{\psi}(\widetilde{\tau}) \otimes \sigma_{a}}\right)$ and obtain

$$
\int_{\left[\mathrm{Sp}_{2 r}\right]} \widetilde{\phi}_{\widetilde{\tau}^{\prime}}(\widetilde{g}) \int_{\left[V_{2 r+l, r}\right]} \widetilde{\theta}_{\varphi_{2 r}}^{\psi}\left(\ell_{2 r}(v) \widetilde{g}\right) E\left(v \widetilde{g}, s, \phi_{\pi_{\psi}(\widetilde{\tau}) \otimes \sigma_{a}}\right) \overline{\psi_{r+l}(v)} d v d \widetilde{g} .
$$

Since the inner integration in integral (6.6) produces an automorphic form on $\widetilde{\mathrm{Sp}}_{2 r}(\mathbb{A})$ and $\widetilde{\phi}_{\widetilde{\tau}^{\prime}}$ is cuspidal, we know that integral (6.6) converges absolutely for the real part of $s$ large and has meromorphic continuation to the whole $\mathbb{C}$-plane. Since integral (6.5) is the residue of integral (6.6) at $s=\frac{1}{2}$, one concludes that integral (6.6) does not vanish for some choice of data when the real part of $s$ is large, because integral (6.5) is not identically zero.

Hence the proof of Theorem 6.3 will be completed by means of Proposition 6.6 below.

Proposition 6.6. If the period integral

$$
\mathcal{P}_{r, r-l}\left(\phi_{\sigma}, \widetilde{\phi}_{\tilde{\tau}}, \varphi_{l}\right)(r \geq l) \quad \text { or } \quad \widetilde{\mathcal{P}}_{l, l-r}\left(\widetilde{\phi}_{\tilde{\tau}}, \phi_{\sigma}, \varphi_{r}\right)(r \leq l)
$$

vanishes identically, then the integral in (6.6) vanishes identically for all choices of data.

Proof. The idea is to unfold integral (6.6), for the real part of $s$ large, and to obtain the above period as inner integration.

For the real part of $s$ large, we unfold the Eisenstein series in (6.6) and we obtain

$$
\sum_{w} \int \widetilde{\phi}_{\widetilde{\tau}_{1}}(\widetilde{g}) \widetilde{\theta}_{\varphi_{2 r}}^{\psi}\left(\ell_{2 r}(v) \widetilde{g}\right) \Phi(w v \widetilde{g}, s, \phi) \overline{\psi_{r+l}(v)} d v d \widetilde{g}
$$

where the summation on $w$ is taken from the set of representatives of double cosets in $P_{2 r, l}(k) \backslash \mathrm{Sp}_{4 r+2 l}(k) / \mathrm{Sp}_{2 r}(k) V_{2 r+l, r}(k)$, and the integration is over $\left(w^{-1} P_{2 r, l}(k) w \cap \operatorname{Sp}_{2 r}(k) V_{2 r+l, r}(k)\right) \backslash \operatorname{Sp}_{2 r}(\mathbb{A}) V_{2 r+l, r}(\mathbb{A})$. Since the double coset decomposition is of generalized Bruhat type, the representatives $w$ can be chosen from the Weyl elements in $\mathrm{Sp}_{4 r+2 l}$.

We shall first show that all the terms in (6.7), except one, vanish by means of the admissibility argument of double cosets. We write $w=\left(w_{i, j}\right)$ where $w_{i, j}$ is the $(i, j)$ th entry of $w$. We may assume that $w_{i, j}$ take values in $\{0, \pm 1\}$. If $v \in V_{2 r+l, r}$ such that $w v w^{-1} \in U_{2 r, l}$ and $\psi_{r+l}(v) \neq 1$, then the summand in (6.7) corresponding to this $w$ is zero, because of the left and right quasi-invariance property of the integral. In this case, we say that $w$ is not admissible.

For $1 \leq i \leq 2 r+l$, let $\alpha_{i}$ denote the $i$-th simple root of $\mathrm{Sp}_{4 r+2 l}$. Let $x_{\alpha_{i}}(c)$ denote the one parameter unipotent subgroup of $S p_{4 r+2 l}$ corresponding to the simple root $\alpha_{i}$. In matrices we have $x_{\alpha_{i}}(c)=I_{4 r+2 l}+c e_{i, i+1}^{\prime}$. Here $e_{i, i+1}^{\prime}=$ 
$e_{i, i+1}-e_{4 r+2 l-i, 4 r+2 l-i+1}$ if $i \leq 2 r+l-1$ and $e_{i, i+1}^{\prime}=e_{i, i+1}$ if $i=2 r+l$. Also denote $x_{r+l}(c)=I_{4 r+2 l}+c e_{r+l, 3 r+l+1}$, which is the one-parameter subgroup of $\mathrm{Sp}_{4 r+2 l}$ attached to the positive root $2 \alpha_{r+l}+\cdots+2 \alpha_{2 r+l-1}+\alpha_{2 r+l}$. Clearly, $x_{\alpha_{i}}(c), x_{r+l}(c) \in V_{2 r+l, r}$ for $1 \leq i \leq r+l-1$. It follows from the definition of $\psi_{r+l, a}$ (as in (2.5) for the case $n=2 r+l$ and $i=r+l$ ) and the left quasi-invariant property of $\widetilde{\theta}_{\varphi_{2 r}}^{\psi}$ that if one of $w x_{\alpha_{i}}(c) w^{-1}$ (for $1 \leq i \leq r+l-1$ ) or $w x_{r+l}(c) w^{-1}$ belongs to $U_{2 r, l}$, then the summand in (6.7) corresponding to $w$ is zero; i.e., $w$ is not admissible.

Let $w=\left(w_{i, j}\right)$ be one of the representatives. We first assume $w_{i, 1}=1$ with $1 \leq i \leq 2 r$. In this case, by multiplying by a Weyl element of $P_{2 r, l}$ on the left, we may obtain a new representative $w=\left(w_{i, j}\right)$ with $w_{1,1}=1$. Next we consider $w_{i, 2}$ for $i>1$. If $i>2 r$, we will have $w x_{\alpha_{1}}(c) w^{-1} \in U_{2 r, l}$ and hence this representative $w$ is not admissible. We obtain that $w_{i, 2}=1$ for some $i \leq 2 r$. In this case, by multiplying on the left by a suitable element of $P_{2 r, l}$, we may obtain a new representative $w=\left(w_{i, j}\right)$ with

$$
w_{1,1}=w_{2,2}=1 \text {. }
$$

Continuing with this argument, we deduce that the representative $w$ is either not admissible or can be chosen to be in the form

$$
w=\left(\begin{array}{ccc}
I_{p} & & \\
& w^{\prime} & \\
& & I_{p}
\end{array}\right)
$$

where $p$ depends on $r$ and $l$ as follows. If $r \geq l$, then $2 r \geq r+l$ and we would have $p=r+l$. But in this case we have $w x_{r+l}(c) w^{-1} \in U_{2 r, l}$; i.e., $w$ is not admissible. If $r<l$, then $2 r<r+l$ and we would have $p=2 r$. In this case we have $w x_{\alpha_{2 r}}(c) w^{-1} \in U_{2 r, l}$. Thus in both cases any representative $w$ such that $w_{i, 1}=1$ with $1 \leq i \leq 2 r$ is not admissible. Note that we may start with $w_{i, 1}=-1$ with $1 \leq i \leq 2 r$, but the positive or negative sign of the representatives is not essential to the admissibility of the representatives.

Next assume $w_{i, 1}=1$ where $2 r+1 \leq i \leq 4 r$. In this case, by multiplying a suitable element in $P_{2 r, l}$ on the left, we may obtain a new representative $w=\left(w_{i, j}\right)$ with $w_{2 r+1,1}=1$. Suppose $w_{i, 2}=1$. If $i>2 r+2 l$, then $w x_{\alpha_{1}}(c) w^{-1} \in U_{2 r, l}$. This implies that the representative is not admissible. If $i \leq 2 r$, we can obtain a new representative $w=\left(w_{i, j}\right)$ with $w_{1,2}=1$ by multiplying a suitable element in $P_{2 r, l}$ on the left. Arguing as above, we deduce that the representative $w$ is either not admissible or has the form

$$
w=\left(\begin{array}{ccccc}
0 & I_{p} & * & 0 & 0 \\
0 & 0 & * & 0 & 0 \\
1 & 0 & * & 0 & 0 \\
0 & 0 & * & 0 & 1 \\
0 & 0 & * & 0 & 0 \\
0 & 0 & * & I_{p} & 0
\end{array}\right)
$$

If $r \geq l$, then $p=r+l$ and $w x_{r+l}(c) w^{-1} \in U_{2 r, l}$, and hence $w$ is not admissible. If $r<l$, then $p=2 r-1$ and $w x_{\alpha_{2 r}}(c) w^{-1} \in U_{2 r, l}$, and hence $w$ is not admissible. Therefore, we deduce that if $w_{i, 2}=1$, then $2 r+2 \leq i \leq 2 r+2 l-1$ (since $\left.w_{2 r+1,1}=1\right)$. Multiplying a suitable element in $P_{2 r, l}$ on the left, we may assume that $i=2 r+2$. Arguing as above, this time with $w_{i, 3}=1$ and so on, we can 
continue this process and deduce that

$$
w=\left(\begin{array}{cccc}
0 & * & * & 0 \\
I_{l} & 0 & 0 & 0 \\
0 & 0 & 0 & I_{l} \\
0 & * & * & 0
\end{array}\right)
$$

But now, if $w_{i, l+1}=1$, then either $i>2 r+2 l$ or $i \leq 2 r$. Hence, as above, we deduce that this $w$ is not admissible.

Thus we may assume that $w_{2 r+2 l+1,1}=1$. Repeating this process with the first $2 r$ columns, we are finally left with a unique element which is

$$
w_{0}=\left(\begin{array}{ccc} 
& & I_{2 r} \\
& I_{2 l} & \\
I_{2 r} & &
\end{array}\right) .
$$

This Weyl element represents an admissible summand in (6.7). The vanishing of this summand follows from the vanishing of the periods.

First assume that $r \leq l$. For $w_{0}$ we have

$$
w_{0}^{-1} P_{2 r, l} w_{0} \cap\left(\mathrm{Sp}_{2 r} V_{2 r+l, r}\right)=N_{2 r} \times\left(\mathrm{Sp}_{2 r} V_{l, r}\right)
$$

where the notation refers to $\S 2$. Thus, integral (6.6) equals in this case

$$
\int \widetilde{\phi}_{\widetilde{\tau}_{1}}(\widetilde{g}) \widetilde{\theta}_{\varphi_{2 r}}^{\psi}\left(\ell_{2 r}(v) \widetilde{g}\right) \Phi(w v \widetilde{g}, s, \phi) \psi_{r+l}(v) d v d g
$$

where we integrate over $N_{2 r}(k) \times\left(\mathrm{Sp}_{2 r} V_{l, r}\right)(k) \backslash \mathrm{Sp}_{2 r}(\mathbb{A}) V_{2 r+l, r}(\mathbb{A})$. Factoring the measure with respect to $\operatorname{Sp}_{2 r} V_{l, r}$, we obtain the period $\widetilde{\mathcal{P}}_{l, l-r}\left(\widetilde{\phi}_{\widetilde{\tau}}, \phi_{\sigma}, \varphi_{r}\right)$ as an inner integral in the above integral.

Next assume that $r>l$. Multiplying by elements in $P_{2 r, l}$ on the left and by elements in $\mathrm{Sp}_{2 r}$ on the right, we may assume that

$$
w_{0}=\left(\begin{array}{lll} 
& & I_{r+l} \\
& I_{2 r} & \\
I_{r+l} & &
\end{array}\right) .
$$

In this case we obtain

$$
w_{0}^{-1} P_{2 r, l} w_{0} \cap\left(\mathrm{Sp}_{2 r} V_{2 r+l, r}\right)=N_{2 r, r-l} \times P_{r-l, l}
$$

where $P_{r-l, l}$ is the standard parabolic subgroup of $\mathrm{Sp}_{2 r}$ whose Levi part is $\mathrm{GL}_{r-l} \times$ $\mathrm{Sp}_{2 l}$ and

$$
N_{2 r, r-l}=\left\{\left(\begin{array}{cc}
v_{1} & v_{2} \\
& I_{r-l}
\end{array}\right) \times I_{2 l} \in V_{2 r+l, r}\right\} .
$$

Here $v_{1} \in N_{r+l}$ and $v_{2} \in \operatorname{Mat}_{(n+k) \times(n-k)}$. Continuing as in the proof of Theorem 5.1 in GRS98, we once again obtain the period $\mathcal{P}_{r, r-l}\left(\phi_{\sigma}, \widetilde{\phi}_{\tilde{\tau}}, \varphi_{l}\right)$ as an inner integration in the above integral.

Hence, by assumption, the above integral is zero for all choices of data. This completes the proof of Proposition 6.6. 
6.1. Local structures. In this subsection we study the structure of the local unramified components of the residue $E_{\frac{1}{2}}\left(g, \phi_{\pi_{\psi}(\widetilde{\tau}) \otimes \sigma_{a}}\right)$ and prove the second assertion stated above Remark 6.4.

Let $F$ be a nonarchimedean local field associated to the number field $k$. Let $\sigma$ be an irreducible admissible generic unramified representation of $\operatorname{Sp}_{2 l}(F)$ and let $\widetilde{\tau}$ be an irreducible admissible generic unramified representation of ${\widetilde{\mathrm{Sp}_{2 r}}}_{2}(F)$. Following GRS99b], we can express $\sigma$ and $\widetilde{\tau}$ as (normalized) induced representations:

$$
\sigma=\operatorname{Ind}_{B}^{\mathrm{Sp}_{2 l}}(\mu) ; \quad \widetilde{\tau}=\operatorname{Ind}_{\widetilde{B}}^{\widetilde{\mathrm{Sp}_{2 r}}}\left(\chi \circ \gamma_{\psi}\right)
$$

respectively, where $\mu$ is a character consisting of $l$ unramified characters $\mu_{1}, \mu_{2}, \cdots$, $\mu_{l}$ of $F^{\times}, \chi$ consists of $r$ unramified characters $\chi_{1}, \chi_{2}, \cdots, \chi_{r}$ of $F^{\times}$, and $\gamma_{\psi}$ is the Weil factor attached to the Weil representation $\omega_{\psi}$. By assumption the local $\psi$ transfer can be defined as ([GRS99b])

$$
\pi_{\psi}(\widetilde{\tau})=\operatorname{Ind}_{B\left(\mathrm{GL}_{2 r}\right)}^{\mathrm{GL}_{2 r}}\left(\chi^{\prime}\right)
$$

where $\chi^{\prime}$ is an unramified character of $\mathrm{GL}_{1}^{2 r}$ defined by

$$
\chi^{\prime}\left(\operatorname{diag}\left(t_{1}, t_{2}, \cdots, t_{r}, t_{r+1}, \cdots, t_{2 r-1}, t_{2 r}\right)\right):=\chi_{1}\left(\frac{t_{1}}{t_{2 r}}\right) \chi_{2}\left(\frac{t_{2}}{t_{2 r-1}}\right) \cdots \chi_{r}\left(\frac{t_{r}}{t_{r+1}}\right) .
$$

Let $Q_{l, r}$ denote the standard parabolic subgroup of $\mathrm{Sp}_{4 r+2 l}$ whose Levi part is $\mathrm{GL}_{1}^{l} \times \mathrm{GL}_{2}^{r}$. By definition $\mu$ depends on $l$ unramified characters $\mu_{i}$ of $F^{\times}$and $\chi$ depends on $r$ unramified characters $\chi_{j}$ of $F^{\times}$. We define an unramified character $(\mu, \chi)$ on $\mathrm{GL}_{1}^{l} \times \mathrm{GL}_{2}^{r}$ as

$$
(\mu, \chi)\left(a_{1}, \cdots, a_{l}, h_{1}, \cdots, h_{r}\right)=\prod_{i=1}^{l} \mu_{i}\left(a_{i}\right) \prod_{j=1}^{r} \chi_{j}\left(\operatorname{det} h_{j}\right)
$$

for all $a_{i} \in \mathrm{GL}_{1}(F)$ and $h_{j} \in \mathrm{GL}_{2}(F)$. We extend $(\mu, \chi)$ canonically to a character of $Q_{l, r}$.

First we have

Lemma 6.7. The local unramified component of the residue $E_{\frac{1}{2}}\left(g, \phi_{\pi_{\psi}(\widetilde{\tau}) \otimes \sigma_{a}}\right)$ is the unique unramified quotient of the (normalized) induced representation

$$
\operatorname{Ind}_{Q_{l, r}}^{\mathrm{Sp}_{4 r+2 l}}(\mu, \chi) \text {. }
$$

Proof. The proof is the same as the proof of Proposition 1 in GRS99b. We omit the details.

Next we proceed as in Sections 2 and 3 in GRS. We recall from (2.1) that for $1 \leq p \leq 2 r+l, V_{2 r+l-p}$ is the unipotent radical of the standard parabolic subgroup of $\mathrm{Sp}_{4 r+2 l}$ whose Levi part is $\mathrm{GL}_{1}^{p} \times \mathrm{Sp}_{4 r+2 l-2 p}$. As in (2.4), $V_{2 r+l-p}$ has a normal subgroup $V_{\mathcal{O}_{((2 p) 14 r+2 l-2 p)}}$. For simplicity we denote $V_{\mathcal{O}_{((2 p) 14 r+2 l-2 p)}}$ by $U_{p}$ in the rest of this section. More precisely, we have

$$
U_{p}=\left\{v=\left(v_{i, j}\right) \in V_{2 r+l-p}: v_{p, j}=0 \text { for all } p+1 \leq j \leq 4 r+2 l-p\right\} .
$$

For $a \in F^{\times}$, characters $\psi_{p}$ and $\psi_{U_{p}}^{a}=\psi_{\left((2 p) 1^{4 r+2 l-2 p)}\right.}^{a}$ are defined in (2.3) and (2.5). Given a representation $\left(\epsilon, X_{\epsilon}\right)$ of $\operatorname{Sp}_{4 r+2 l}(F)$, we let $\mathcal{L}_{p, a}$ be a linear functional on the space $X_{\epsilon}$ satisfying

for all $u \in U_{p}$ and $x \in X_{\epsilon}$.

$$
\mathcal{L}_{p, a}(u x)=\psi_{U_{p}}^{a}(u) \mathcal{L}_{p, a}(x)
$$


As in Lemma 2 in GRS we state

Lemma 6.8. Let $X_{\pi_{\psi}(\widetilde{\tau}) \otimes \sigma_{a}, v}$ be the local unramified component of the residue representation $E_{\frac{1}{2}}\left(g, \phi_{\pi_{\psi}(\widetilde{\tau}) \otimes \sigma_{a}}\right)$ at a finite local place $v\left(F=k_{v}\right)$. Then for all $a \in$ $F^{\times}$the representation $X_{\pi_{\psi}(\widetilde{\tau}) \otimes \sigma_{a}, v}$ has no nonzero functional $\mathcal{L}_{p, a}$ for $p=r+l+1$.

Proof. This is done exactly as in the proof of Lemma 2 in GRS. We sketch the idea of the proof. By Lemma 6.7 it is enough to prove Lemma 6.8 for $\operatorname{Ind}_{Q_{l, r}}^{\operatorname{Sp}_{4 r+2 l}}(\mu, \chi)$. This is done by the Mackey theory. In other words we need to consider the space $Q_{l, r} \backslash \mathrm{Sp}_{4 r+2 l} / U_{r+l+1}$ and show that for every representative $\gamma$ of the double cosets, there exists $u \in U_{r+l+1}$ such that $\gamma u \gamma^{-1} \in Q_{l, r}$ and $\psi_{U_{r+l+1}}^{a}(u) \neq 1$. This is done as in the proof of Lemma 2 of [GRS. We omit the details here.

Finally, we sketch the proof of the second assertion above Remark 6.4. If $\widetilde{\tau}^{\prime}$ is an irreducible summand of $\widetilde{\tau}_{a}$, then arguing as in [GRS], we show that the unramified local component of $\widetilde{\tau}^{\prime}$ is given by an induced representation

$$
\operatorname{Ind}_{\widetilde{B}\left(k_{v}\right)}^{\widetilde{\operatorname{Sp}_{2 r}}\left(k_{v}\right)}\left(\chi_{v} \circ \gamma_{\psi_{v}}\right)
$$

and that $\gamma_{\psi_{v}}=\gamma_{\psi}$ and $\chi_{v}$ is equal to $\chi$ up to a twist by a Weyl element. Recall that $\gamma_{\psi}$ and $\chi$ are defined in (6.8) and (6.9). This means that the local unramified component of $\widetilde{\tau}^{\prime}$ is equivalent to the corresponding local unramified component of $\widetilde{\tau}$. This proves the second assertion. The detail of the argument can be found in the proof of Proposition 5 in [GRS].

\section{On Assumption (FC)}

In this section we shall prove Assumption (FC) for the case $l=1$, while for the case $l=0$, it is proved in GRS99a]. In other words, we shall prove

Proposition 7.1. Let $E_{\frac{1}{2}}\left(g, \phi_{\pi_{\psi}(\widetilde{\tau}) \otimes \sigma}\right)$ be the residue at $s=\frac{1}{2}$ of the Eisenstein series $E\left(g, s, \phi_{\pi_{\psi}(\widetilde{\tau}) \otimes \sigma}\right)$ on $\mathrm{Sp}_{4 r+2}(\mathbb{A})$ as defined in (3.10). If the residue $E_{\frac{1}{2}}\left(g, \phi_{\pi_{\psi}(\widetilde{\tau}) \otimes \sigma}\right)$ is nonzero, then it has a nonzero Fourier coefficient associated to the unipotent class parameterized by the symplectic partition $\left((2 r+2) 1^{2 r}\right)$.

Proof. Let $U$ denote the unipotent radical subgroup of the standard parabolic subgroup of $\mathrm{Sp}_{4 r+2}$ whose Levi part is $\mathrm{GL}_{2}^{r-1} \times \mathrm{GL}_{3}$. We identify $U /[U, U]$ with the affine space

$$
\mathcal{A}_{U}=\left(\bigoplus_{i=1}^{r-2} \mathrm{Mat}_{2 \times 2}\right) \oplus \mathrm{Mat}_{2 \times 3} \oplus \mathrm{Mat}_{3 \times 3}^{\prime}
$$

where Mat $_{i \times j}$ denotes the space of all $i \times j$-matrices, and

$$
\operatorname{Mat}_{j \times j}^{\prime}=\left\{X \in \operatorname{Mat}_{j \times j}: w_{j} X^{t}=X w_{j}\right\}
$$

where $w_{j}$ is the longest Weyl element of $\mathrm{GL}_{j}$. To define characters on $U$, we denote elements of $\mathcal{A}_{U}$ by

$$
u=u\left(z_{1}, \cdots, z_{r-2}, x, y\right) \in \mathcal{A}_{U}
$$

where $z_{i} \in \operatorname{Mat}_{2 \times 2}, x=\left(x_{i, j}\right) \in \operatorname{Mat}_{2 \times 3}$, and $y=\left(y_{i, j}\right) \in \operatorname{Mat}_{3 \times 3}^{\prime}$. For $a \in k^{\times}$, we define a character $\psi_{U}^{a}$ on $U$ by

$$
\psi_{U}^{a}(u)=\psi\left(\operatorname{tr}\left(z_{1}\right)+\cdots+\operatorname{tr}\left(z_{r-2}\right)+x_{1,1}+x_{2,3}+y_{1,1}+a y_{2,2}\right) .
$$


Define the function

$$
f(g)=\int_{U(F) \backslash U(\mathbb{A})} E_{\frac{1}{2}}\left(u g, \phi_{\pi_{\psi}(\widetilde{\tau}) \otimes \sigma} \overline{\psi_{U}^{a}(u)} d u .\right.
$$

As explained in GRS03, this is a Fourier coefficient corresponding to the unipotent

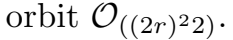

Let $\beta$ denote the Weyl element of $\mathrm{GL}_{2 r+1}$ defined by

$$
\beta=\left(\left(\begin{array}{ll}
0 & 1 \\
1 & 0
\end{array}\right), \cdots,\left(\begin{array}{ll}
0 & 1 \\
1 & 0
\end{array}\right),\left(\begin{array}{lll}
0 & 0 & 1 \\
0 & 1 & 0 \\
1 & 0 & 0
\end{array}\right)\right) \in \mathrm{GL}_{2}^{r-1} \times \mathrm{GL}_{3}
$$

and let $\beta_{0}=m(\beta)=\left(\begin{array}{cc}\beta & \\ & \beta^{*}\end{array}\right) \in \operatorname{Sp}_{4 r+2}$. It is easy to check that $f\left(\beta_{0} g\right)=f(g)$.

Let $t(b)$, for $b \in \mathbb{A}^{\times}$, denote the torus element in $\mathrm{GL}_{2}^{r-1} \times \mathrm{GL}_{3}$ defined by

$$
t(b)=\left(\left(\begin{array}{ll}
b & \\
& b^{-1}
\end{array}\right), \cdots,\left(\begin{array}{ll}
b & \\
& b^{-1}
\end{array}\right),\left(\begin{array}{ccc}
b & & \\
& 1 & \\
& & b^{-1}
\end{array}\right)\right) \in \mathrm{GL}_{2}^{r-1} \times \mathrm{GL}_{3} .
$$

Then $m(t(b))$ is a diagonal element in $\operatorname{Sp}_{4 r+2}(\mathbb{A})$. It is clear that

$$
\beta_{0} t(b) \beta_{0}^{-1}=t\left(b^{-1}\right) .
$$

The idea of the proof is to show that if the residue $E_{\frac{1}{2}}\left(g, \phi_{\pi_{\psi}(\widetilde{\tau}) \otimes \sigma}\right)$ has no nonzero Fourier coefficients associated to the unipotent orbit $\mathcal{O}_{\left((2 r+2) 1^{2 r}\right)}$, then the following two statements hold.

(1) The function $f(g)$ defined in (7.3) is not identically zero.

(2) For all $b \in \mathbb{A}^{\times}, f(m(t(b)) g)=|b|_{\mathbb{A}} f(g)$.

On the other hand, statements (1) and (2) lead to a contradiction. Indeed, using (2) and the left invariance of $f(g)$ by $\beta_{0}$, it is easy to show the identity

$$
|b|_{\mathbb{A}} f(g)=f(m(t(b)) g)=f\left(\beta_{0} m(t(b)) g\right)=f\left(m\left(t\left(b^{-1}\right)\right) \beta_{0} g\right)=|b|_{\mathbb{A}}^{-1} f(g)
$$

for all $b \in \mathbb{A}^{\times}$and all $g \in \operatorname{Sp}_{4 r+2}(\mathbb{A})$. Since $b$ and $g$ are arbitrary, the function $f(g)$ must be identically zero, which contradicts statement (1). This proves Proposition 7.1 .

It remains to show that the vanishing of the Fourier coefficients of the residue $E_{\frac{1}{2}}\left(g, \phi_{\pi_{\psi}(\widetilde{\tau}) \otimes \sigma}\right)$ associated to the unipotent orbit $\mathcal{O}_{\left((2 r+2) 1^{2 r}\right)}$ implies statements (1) and (2) above.

We define a Weyl element $w=\left(w_{i, j}\right)$ of $\mathrm{Sp}_{4 r+2}$. In term of matrices we can choose Weyl elements of $\mathrm{Sp}_{4 r+2}$ to have entries belonging to the set $\{0,1,-1\}$. With this convention, to determine a Weyl element in $\mathrm{Sp}_{4 r+2}$ completely, it is enough to specify its entries in the first $2 r+1$ rows. The Weyl element we need to define is as follows. For $1 \leq i \leq r$ we set $w_{i, 2 i-1}=1$. Also $w_{r+1,2 r+2}=1$ and for $2 \leq i \leq r$ we set $w_{r+i, 2 r+2 i+1}=1$. Finally we set $w_{2 r+1,2 r}=1$. All other entries in the first $2 r+1$ rows of $w$ are zeroes. One can check that this Weyl element $w$ is the one that conjugates the character $\psi_{U}^{a}$ in (7.2) to the character $\psi_{a}$ stated in (7.7).

In (7.3), since the residue $E_{\frac{1}{2}}\left(g, \phi_{\pi_{\psi}(\widetilde{\tau}) \otimes \sigma}\right)$ is automorphic, we conjugate the Weyl element $w$ from the left to the right across the integration and obtain that $f(g)$ 
equals

$$
\int E_{\frac{1}{2}}\left(\left(\begin{array}{ccc}
v_{1} & x & y \\
& v_{2} & x^{*} \\
& & v_{1}^{*}
\end{array}\right)\left(\begin{array}{ccc}
I_{2 r} & & \\
p & I_{2} & \\
q & p^{*} & I_{2 r}
\end{array}\right) w g, \phi_{\pi_{\psi}(\widetilde{\tau}) \otimes \sigma}\right) \psi_{a}\left(\left(v_{1}, v_{2}\right)\right) d(\ldots) .
$$

Here $v_{1} \in N_{2 r}(k) \backslash N_{2 r}(\mathbb{A})$ ( $N_{2 r}$ is the standard maximal unipotent subgroup of $\left.\mathrm{GL}_{2 r}\right), v_{2} \in N_{2}(k) \backslash N_{2}(\mathbb{A})$. Let

$$
\mathcal{Z}_{1}=\left\{z \in \text { Mat }_{2 r \times 2 r}: z_{i, j}=0 \text { for all } i \geq j\right\} .
$$

We integrate variables $y$ and $q$ over matrices in $\mathcal{Z}_{1}$. The variable $x$ is integrated over all $2 r \times 2$ matrices such that $x_{i, j}=0$ if $i \geq r$ and also $x_{r-1,1}=0$. This matrix subgroup is denoted by $\mathcal{Z}_{3}$. The variable $p$ is integrated over all matrices of size $2 \times 2 r$ such that $p_{i, j}=0$ if $j \leq r$ and $p_{2, r+1}=0$. We shall denote this subgroup by $\mathcal{Z}_{2}$. Each of these variables is integrated over the quotient $\mathcal{Z}_{i}(k) \backslash \mathcal{Z}_{i}(\mathbb{A}), i=1,2,3$, respectively. Finally, we have

$$
\psi_{a}\left(\left(v_{1}, v_{2}\right)\right)=\psi\left(\left(v_{1}\right)_{1,2}+\cdots+\left(v_{1}\right)_{2 r-1,2 r}+a\left(v_{2}\right)_{1,2}\right) .
$$

Following the proof of Theorem 1 in GRS99a], we consider certain Fourier expansions for the integral in (7.6). Let

$$
L=\left\{\left(\begin{array}{ccc}
I_{2 r} & \ell_{1} & \ell_{2} \\
& I_{2} & \ell_{1}^{*} \\
& & I_{2 r}
\end{array}\right) \in \operatorname{Sp}_{4 r+2}\right\}
$$

where $\ell_{1} \in M a t_{2 r \times 2}$ such that $\left(\ell_{1}\right)_{i, j}=0$ except at the $(r-1,1)$-th entry and

$$
\ell_{2}=\left\{\left(\begin{array}{cc}
l_{2} & 0 \\
0 & l_{2}^{*}
\end{array}\right):\left(l_{2}\right)_{i, j}=0 \text { for all } i<j\right\} .
$$

Note that $L$ is abelian. In (7.6) we expand the integral along $L(k) \backslash L(\mathbb{A})$. Let

$$
T=\left\{\left(\begin{array}{ccc}
I_{2 r} & & \\
t_{2} & I_{2} & \\
t_{1} & t_{2}^{*} & I_{2 r}
\end{array}\right) \in \mathrm{Sp}_{4 r+2}\right\}
$$

where $t_{1} \in$ Mat $_{2 \times 2 r}$ such that $\left(t_{1}\right)_{i, j}=0$ except at the $(1, r)$ position and

$$
t_{2}=\left\{q \in \mathcal{Z}_{1}: q_{i, j}=0 \text { for all } 1 \leq i \leq r-1 ; r+1 \leq j \leq 2 r\right\} .
$$

We denote

$$
\mathcal{T}_{1} \times \mathcal{T}_{2}:=\left\{\left(t_{1}, t_{2}\right) \mid\left(\begin{array}{ccc}
I_{2 r} & & \\
t_{2} & I_{2} & \\
t_{1} & t_{2}^{*} & I_{2 r}
\end{array}\right) \in T\right\} .
$$

Then we know that $\mathcal{T}_{1} \times \mathcal{T}_{2}$ is a subgroup of $\mathcal{Z}_{1} \times \mathcal{Z}_{2}$. Conjugating from left to right by the matrices in $T(k)$ and collapsing the summation with integration, we deduce that $f(g)$ equals

$$
\int E_{\frac{1}{2}}\left(\left(\begin{array}{ccc}
v_{1} & x & y \\
& v_{2} & x^{*} \\
& & v_{1}^{*}
\end{array}\right)\left(\begin{array}{ccc}
I_{2 r} & & \\
p & I_{2} & \\
q & p^{*} & I_{2 r}
\end{array}\right)\left(\begin{array}{ccc}
I_{2 r} & & \\
t_{1} & I_{2} & \\
t_{2} & t_{1}^{*} & I_{2 r}
\end{array}\right) w g, \phi\right) \psi_{a}\left(\left(v_{1}, v_{2}\right)\right) d(\ldots)
$$

where $\phi=\phi_{\pi_{\psi}(\widetilde{\tau}) \otimes \sigma}$. The integrations over $v_{1}$ and $v_{2}$ are the same as in (7.6). The variables $t_{1}$ and $t_{2}$ are integrated over $\mathcal{T}_{1}(\mathbb{A}) \times \mathcal{T}_{2}(\mathbb{A})$ and $p$ and $q$ are integrated over $\left(\mathcal{Z}_{1} \times \mathcal{Z}_{2}\right)(k)\left(\mathcal{T}_{1} \times \mathcal{T}_{2}\right)(\mathbb{A}) \backslash\left(\mathcal{Z}_{1} \times \mathcal{Z}_{2}\right)(\mathbb{A})$. The variable $x$ is integrated over all 
matrices of size $2 r \times 2$ such that $x_{i, j}=0$ for $i \geq r+1$ and $y$ is integrated over all matrices of the form

$$
\left\{w_{2 r} y^{t}=y w_{2 r}: y_{i, j}=0 \text { if } i \geq r+1 \text { and } j \leq r-1\right\} .
$$

More precisely, the integrations in $x$ and $y$ are over the quotient of the $\mathbb{A}$-rational points over the $k$-rational points of the corresponding algebraic groups consisting of all matrices as described above, respectively.

In the following we consider the Fourier expansion of the above integral along the one-parameter additive subgroup $\chi_{\alpha}(\cdot)$ attached to a positive long root $\alpha$, which consists of all matrices of the form $\left\{I_{4 r+2}+c e_{r+1,3 r+2}\right\}$ where $c \in k$ and $e_{i, j}$ is the $(4 r+2) \times(4 r+2)$ matrix with one at the $(i, j)$-th entry and zero elsewhere. The nontrivial Fourier coefficient of the function defined in (7.8) along the quotient $\chi_{\alpha}(k) \backslash \chi_{\alpha}(\mathbb{A})$ is zero. Indeed, the integrations along variables $v_{1}, x, y$ and $\chi_{\alpha}$ in the integral in (7.8) (against the nontrivial character $\psi_{a}\left(v_{1}\right) \psi(b c)$ for some $b \in k^{\times}$) yield the Fourier coefficient of the residue $E_{\frac{1}{2}}\left(g, \phi_{\pi_{\psi}(\widetilde{\tau}) \otimes \sigma}\right)$ attached to the unipotent orbit $\mathcal{O}_{\left((2 r+2) 1^{2 r}\right)}$. By our assumption this Fourier coefficient is zero. This means that in the Fourier expansion of (7.8) along $\chi_{\alpha}(k) \backslash \chi_{\alpha}(\mathbb{A})$ only the trivial character (or the constant term) contributes.

Continuing this process as in the proof of Theorem 1 in GRS99a, we finally obtain that the function $f(g)$ is equal to

$$
\left.\int_{\left(\mathcal{Z}_{1} \times \mathcal{Z}_{2}\right)(\mathbb{A})} \int E_{\frac{1}{2}}^{U_{2 r, 1}}\left(m\left(v_{1}, v_{2}\right)\left(\begin{array}{ccc}
I_{2 r} & & \\
p & I_{2} & \\
q & p^{*} & I_{2 r}
\end{array}\right) w g, \phi\right) \psi_{a}\left(v_{1}, v_{2}\right)\right) d(\cdots) .
$$

Here $U_{2 r, 1}$ is the unipotent radical of the parabolic subgroup $P_{2 r, 1}$ of $\mathrm{Sp}_{4 r+2}$ whose Levi part is $\mathrm{GL}_{2 r} \times \mathrm{Sp}_{2}$ and $E_{\frac{1}{2}}^{U_{2 r, 1}}\left(g, \phi_{\pi_{\psi}}(\widetilde{\tau}) \otimes \sigma\right)$ is the constant term of the residue $E_{\frac{1}{2}}\left(g, \phi_{\pi_{\psi}(\widetilde{\tau}) \otimes \sigma}\right)$ along $P_{2 r, 1}$. In the same way as in the proofs of Lemmas 1 and 2 on pages 895-896 in GRS99a], we deduce that if $f(g)$ is zero for all choices of the data, then the following integral

$$
\int E_{\frac{1}{2}}^{U_{2 r, 1}}\left(m\left(v_{1}, v_{2}\right) g, \phi_{\pi_{\psi}(\widetilde{\tau}) \otimes \sigma}\right) \psi_{a}\left(v_{1}, v_{2}\right) d v_{1} d v_{2}
$$

is zero for all choices of the data. In (7.10), the integration along the variable $v_{1}$ is the same as taking the Whittaker-Fourier coefficient of $\phi_{\pi_{\psi}(\widetilde{\tau})}$ and the integration along the variable $v_{2}$ is the same as taking the Whittaker-Fourier coefficient of $\phi_{\sigma}$. If we choose $a \in k^{\times}$such that $\sigma$ has a nonzero $\psi^{a}$-Whittaker-Fourier coefficient, then the above integral represents a nonzero Whittaker-Fourier coefficient of the cusp forms in the generic cuspidal datum $\left(P_{2 r, 1}, \pi_{\psi}(\widetilde{\tau}) \otimes \sigma\right)$. Thus the function $f(g)$ defined in (7.3) is not identically zero and statement (1) is proved.

To prove statement (2), we first notice that

$$
w m(t(b)) w^{-1}=\operatorname{diag}\left(b, \cdots, b, 1,1, b^{-1}, \cdots, b^{-1}\right) \in \mathrm{Sp}_{4 r+2}
$$

is in the center of the Levi subgroup $\mathrm{GL}_{2 r} \times \mathrm{GL}_{1}$. Then we replace $g$ by $m(t(b)) g$ in integral (7.9) and conjugate it to the left. By changing variables in $\mathcal{Z}_{1}(\mathbb{A})$ and $\mathcal{Z}_{2}(\mathbb{A})$, we obtain the factor $|b|_{\mathbb{A}}^{-\left(2 r^{2}+2 r-1\right)}$. Using the fact that $\pi_{\psi}(\tilde{\tau})$ has a trivial central character, we obtain a factor of $|b|_{\mathbb{A}}^{2 r(r+1)}$ from the left quasi-invariance properties of $E_{\frac{1}{2}}^{U_{2 r, 1}}\left(g, \phi_{\pi_{\psi}(\widetilde{\tau}) \otimes \sigma}\right)$. Thus we obtain

$$
f(m(t(b)) g)=|b|_{\mathbb{A}}^{-\left(2 r^{2}+2 r-1\right)+2 r(r+1)} f(g)=|b|_{\mathbb{A}} f(g) .
$$


This proves statement (2) and hence the proposition follows.

Remark 7.2. (1) The way we proved the nonvanishing of the function $f(g)$ relies on the vanishing assumption of the Fourier coefficient of the residue $E_{\frac{1}{2}}\left(g, \phi_{\pi_{\psi}(\widetilde{\tau}) \otimes \sigma}\right)$ attached to the unipotent orbit $\mathcal{O}_{\left((2 r+2) 1^{2 r}\right)}$. However, we point out that the nonvanishing of $f(g)$ may be proved without this assumption.

(2) This proof of Proposition 7.1 can be generalized. Indeed, if $r \geq l$, we can show that the residue $E_{\frac{1}{2}}\left(g, \phi_{\pi_{\psi}(\widetilde{\tau}) \otimes \sigma}\right)$ has a nonzero Fourier coefficient attached to the unipotent orbit $\mathcal{O}_{\left((2 r+2) 1^{2(r+l-1)}\right)}$. Clearly, if $l>1$, this is not enough to verify Assumption (FC).

\section{ACKNOWLEDGMENT}

We would like to thank M. Harris for his useful comments and suggestions on the earlier version of this paper, and we thank the referee for his/her very helpful suggestions and remarks.

\section{REFERENCES}

[A78] Arthur, J. A trace formula for reductive groups. I. Terms associated to classes in $G(Q)$. Duke Math. J. 45 (1978), no. 4, 911-952. MR 80d:10043

[A80] Arthur, J. A trace formula for reductive groups. II. Applications of a truncation operator. Compositio Math. 40 (1980), no. 1, 87-121. MR 81b:22018

[BFSP04] Boecherer, S.; Furusawa, M.; Schulze-Pillot, R. On the global Gross-Prasad conjecture for Yoshida liftings. Contributions to Automorphic Forms, Geometry, and Number Theory. The Johns Hopkins University Press, 2004.

[CKPSS01] Cogdell, J.; Kim, H.; Piatetski-Shapiro, I.; Shahidi, F. On lifting from classical groups to $\mathrm{GL}_{N}$. Inst. Hautes Études Sci. Publ. Math. No. 93 (2001), 5-30. MR 2002i:11048

[CKPSS] Cogdell, J.; Kim, H.; Piatetski-Shapiro, I.; Shahidi, F. Functoriality for the classical groups. To appear in Inst. Hautes Études Sci. Publ. Math.

[CPS04] Cogdell, J.; Piatetski-Shapiro, I. Remarks on Rankin-Selberg Convolutions. Contributions to Automorphic Forms, Geometry, and Number Theory. The Johns Hopkins University Press, 2004.

[CM93] Collingwood, D.; McGovern, W. Nilpotent orbits in semisimple Lie algebras. Van Nostrand Reinhold Mathematics Series. Van Nostrand Reinhold Co., New York, 1993. MR 94j:17001

[FJ93] Friedberg, S.; Jacquet, H. Linear periods. J. Reine Angew. Math. 443 (1993), 91-139. MR 94k:11058

[F95] Furusawa, M. On the theta lift from $\mathrm{SO}_{2 n+1}$ to $\widetilde{\mathrm{Sp}_{n}}$. J. Reine Angew. Math. 466 (1995), 87-110. MR 96g:11052

[GPSR87] Gelbart, S.; Piatetski-Shapiro, I.; Rallis, S. Explicit Constructors of Automorphic L-Functions. Springer Lecture Notes, Vol. 1254, 1987. MR 89k:11038

[GJR01] Ginzburg, D.; Jiang, D.; Rallis, S. Nonvanishing of the central critical value of the third symmetric power L-functions. Forum Math. 13 (2001), no. 1, 109-132. MR 2001m:11083

[GJR03] Ginzburg, D.; Jiang, D.; Rallis, S. Periods of residue representations of $\mathrm{SO}_{2 l}$. Accepted by Manuscripta Math. 2003.

[GJR] Ginzburg, D.; Jiang, D.; Rallis, S. Non-vanishing of the Central Value of the RankinSelberg L-functions. II. To appear in the volume commemorating the sixtieth birthday of Stephen Rallis.

[GRS98] Ginzburg, D.; Rallis, S; Soudry, D. L-Functions for Symplectic Groups. Bull. Soc. Math. France, 126 (1998), no. 2, 181-244. MR 2000b:22017

[GRS99a] Ginzburg, D.; Rallis, S; Soudry, D. On the correspondence between cuspidal representations of $\mathrm{GL}_{2 n}$ and ${\widetilde{\mathrm{Sp}_{2 n}}}_{2 n}$. J. Amer. Math. Soc. 12 (1999), no.3, 849-907. MR 2000b:22018 
[GRS99b] Ginzburg, D.; Rallis, S; Soudry, D. Lifting Cusp Forms on $\mathrm{GL}_{2 n}$ to $\widetilde{\mathrm{Sp}}_{2 n}$ : The Unramified Correspondence. Duke Math. J. 100 (1999), no. 2, 243-266. MR|2001a:11085

[GRS99c] Ginzburg, D.; Rallis, S; Soudry, D. On explicit lifts of cusp forms from $\mathrm{GL}_{m}$ to classical groups. Ann. of Math. (2) 150 (1999), no. 3, 807-866. MR 2001b:11040

[GRS01] Ginzburg, D.; Rallis, S; Soudry, D. Generic automorphic forms on $\mathrm{SO}(2 n+1)$ : functorial lift to GL(2n), endoscopy, and base change. Internat. Math. Res. Notices 2001, no. 14, 729-764. MR 2002g:11065

[GRS02] Ginzburg, D.; Rallis, S; Soudry, D. Endoscopic representations of ${\widetilde{\mathrm{Sp}_{2 n}}}_{2 n}$. Journal of the Inst. of Math. Jussieu, 1 (2002), no. 1, 77-123. MR 2004c:11074

[GRS03] Ginzburg, D.; Rallis, S; Soudry, D. On Fourier Coefficients of Automorphic Forms of Symplectic Groups. Manuscripta Math. 111 (2003), no. 1, 1-16.

[GRS] Ginzburg, D.; Rallis, S; Soudry, D. Constructions of CAP representations for symplectic groups using the descent method. To appear in the volume commemorating the sixtieth birthday of Stephen Rallis.

[GP92] Gross, B.; Prasad, D. On the decomposition of a representation of $\mathrm{SO}_{n}$ when restricted to $\mathrm{SO}_{n-1}$. Canad. J. Math. 44 (1992), no. 5, 974-1002. MR 93j:22031

[GP94] Gross, B.; Prasad, D. On irreducible representations of $\mathrm{SO}_{2 n+1} \times \mathrm{SO}_{2 m}$. Canad. J. Math. 46 (1994), no. 5, 930-950. MR 96c:22028

[H94] Harris, M. Hodge-de Rham structures and periods of automorphic forms. Motives (Seattle, WA, 1991), 573-624, Proc. Sympos. Pure Math., 55, Part 2, Amer. Math. Soc., Providence, RI, 1994. MR 95e:11058

[HK91] Harris, M.; Kudla, S. The central critical value of a triple product L-function. Ann. of Math. (2) 133 (1991), no. 3, 605-672. MR 93a:11043

[HK92] Harris, M.; Kudla, S. Arithmetic automorphic forms for the nonholomorphic discrete series of GSp(2). Duke Math. J. 66 (1992), no. 1, 59-12. MR 93e:22023

[HK04] Harris, M.; Kudla, S. On a conjecture of Jacquet. Contributions to Automorphic Forms, Geometry, and Number Theory. The Johns Hopkins University Press, 2004.

[194] Ikeda, Tamotsu. On the theory of Jacobi forms and Fourier-Jacobi coefficients of Eisenstein series. J. Math. Kyoto Univ. 34 (1994), no. 3, 615-636. MR 95h:11044

[IS00] Iwaniec, H.; Sarnak, P. Perspectives on the analytic theory of L-functions. GAFA 2000 (Tel Aviv, 1999). Geom. Funct. Anal. 2000, Special Volume, Part II, 705-741. MR 2002b:11117

[JPSS83] Jacquet, H.; Piatetski-Shapiro, I.; Shalika, J. Rankin-Selberg convolutions. Amer. J. Math. 105 (1983), no. 2, 367-464. MR 85g:11044

[JR92] Jacquet, H.; Rallis, S. Symplectic periods. J. Reine Angew. Math. 423 (1992), 175197. MR 93b:22035

[JLR04] Jacquet, H.; Lapid, E.; Rallis, S. A spectral identity for skew symmetric matrices. Contributions to Automorphic Forms, Geometry, and Number Theory. The Johns Hopkins University Press, 2004.

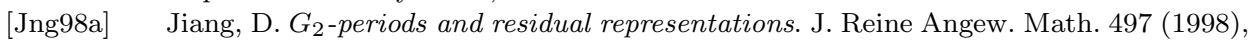
17-46. MR 99e:11062

[Jng98b] Jiang, D. Nonvanishing of the central critical value of the triple product L-functions. Internat. Math. Res. Notices 1998, no. 2, 73-84. MR 99a:11060

[Jng01] Jiang, D. On Jacquet's conjecture: the split period case. Internat. Math. Res. Notices 2001, no. 3, 145-163. MR 2002c:11054

[JS03] Jiang, D.; Soudry, D. The local converse theorem for $\mathrm{SO}(2 n+1)$ and applications. Ann. of Math. (2) 157 (2003), no. 3, 743-806.

[JS04] Jiang, D.; Soudry, D. Generic Representations and the Local Langlands Reciprocity Law for p-adic $\mathrm{SO}(2 n+1)$. Contributions to Automorphic Forms, Geometry, and Number Theory. The Johns Hopkins University Press, 2004.

[K00] Kim, H. Langlands-Shahidi methods and poles of automorphic L-functions II. Israel J. of Math. 117 (2000), 261-284. MR 2001i:11059a

[K02] Kim, H. Applications of Langlands' functoriality of odd orthogonal groups. Trans. AMS. 354 (2002), no. 7, 2775-2796. MR 2003c:22025

[L71] Langlands, R. Euler products. A James K. Whittemore Lecture in Mathematics given at Yale University, 1967. Yale Mathematical Monographs, 1. Yale University Press, New Haven, Conn.-London, 1971. MR 54:7387 
[Lp03] Lapid, E. On the non-negativity of Rankin-Selberg L-functions at the center of symmetry. Internat. Math. Res. Notices 2003, no. 2, 65-75. MR 2003j:11054

[LR03] Lapid, E.; Rallis, S. On the non-negativity of $L\left(\frac{1}{2}, \pi\right)$ for $\mathrm{SO}_{2 n+1}$. Ann. of Math. (2) 157 (2003), no. 3, 891-917. MR 2004d:11039

[MVW87] Moeglin, C.; Vignéras, M.-F.; Waldspurger, J.-L. Correspondances de Howe sur un corps p-adique. (French) Lecture Notes in Mathematics, 1291. Springer-Verlag, Berlin, 1987. MR 91f: 11040

[MW87] Moeglin, C.; Waldspurger, J.-L. Modèles de Whittaker dégénérés pour des groupes p-adiques. (French) Math. Z. 196 (1987), no. 3, 427-452. MR 89f:22024

[MW89] Moeglin, C.; Waldspurger, J.-L. Le spectre résiduel de GL(n). (French) Ann. Sci. École Norm. Sup. (4) 22 (1989), no. 4, 605-674. MR 91b:22028

[MW95] Moeglin, C.; Waldspurger, J.-L. Spectral decomposition and Eisenstein series. Cambridge University Press, 1995. MR 97d:11083

[PS71] Piatetski-Shapiro, I. Euler subgroups. Lie groups and their representations (Proc. Summer School, Bolyai Janos Math. Soc., Budapest, 1971), pp. 597-620. Halsted, New York, 1975. MR 53:10720

[R94] Ramakrishnan, D. Pure motives and automorphic forms. Motives (Seattle, WA, 1991), 411-446, Proc. Sympos. Pure Math., 55, Part 2, Amer. Math. Soc., Providence, RI, 1994. MR 94m:11134

[Shd84] Shahidi, F. Fourier transforms of intertwining operators and Plancherel measures for GL(n). Amer. J. Math. 106 (1984), no. 1, 67-111. MR 86b:22031

[Shd88] Shahidi, F. On the Ramanujan conjecture and the finiteness of poles of certain Lfunctions. Ann. of Math. (2) 127 (1988), no. 3, 547-584. MR 89h:11021

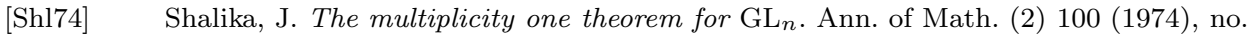
2, 171-193. MR 50:545

[S02] Soudry, D. Langlands functoriality from classical groups to $G L_{n}$. To appear in Asterisque.

[W85] Waldspurger, J.-L. Sur les valeurs de certaines fonctions L automorphes en leur centre de symétrie. (French) Compositio Math. 54 (1985), no. 2, 173-242. MR 87g:11061b

School of Mathematical Sciences, Sackler Faculty of Exact Sciences, Tel Aviv UniVersity, Ramat-Aviv, 69978 IsRael

E-mail address: ginzburg@post.tau.ac.il

School of Mathematics, University of Minnesota, Minneapolis, Minnesota 55455

E-mail address: dhjiang@math.umn.edu

Department of Mathematics, The Ohio State University, Columbus, Ohio 43210

E-mail address: haar@math.ohio-state.edu 